\title{
Parametric Investigation on Thrust Development of a Piezoelectric Transformer \\ Plasma Propulsion System
}

A Thesis

Presented to

The Faculty of the Graduate School

At the University of Missouri

In Partial Fulfillment

Of the Requirements for the Degree

of Master of Science

by

CPT Karl M. Olson

Dr. Scott Kovaleski, Thesis Supervisor

July 2013 
The undersigned, appointed by the Dean of the Graduate School, have examined the dissertation entitled:

\section{Parametric Investigation on Thrust Development of a Piezoelectric Transformer \\ Plasma Propulsion System}

Presented by Karl M. Olson

a candidate for the degree of Master of Science

and hereby certify that in their opinion it is worthy of acceptance.

Dr. Scott Kovaleski

Dr. Naz Islam

Dr. Craig Kluever 


\section{Acknowledgements}

I would like to thank:

My wife, Rose, for her patience and understanding on my need for long nights and hard days while she cared for our son, Silas.

Dr. Scott Kovaleski, for allowing me the opportunity to work in his lab, the freedom to explore, and the guidance to succeed.

James Van Gordon, for delaying his dissertation yet another year, so he could take the time to review and edit my work.

Peter Norgard, for those "check this out" moments and for shooting down all my attempts to develop new theories for science. For always providing solid recommendations on procedures, experiments and anything si-entistic.

Emily Baxter, for proving the explosive capacity of hydrogen... and help on numerous homeworks.

I must acknowledge:

Brady Gall, for fully introducing me to the Millennial Generation. God have mercy on us all. [40] 


\section{CONTENTS}

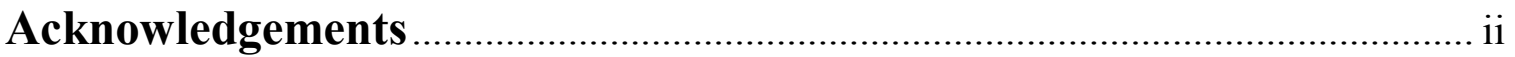

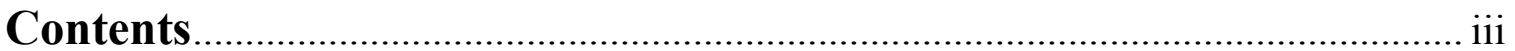

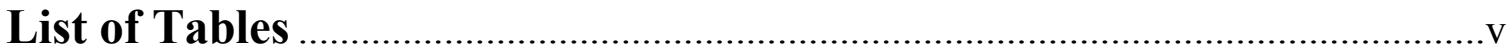

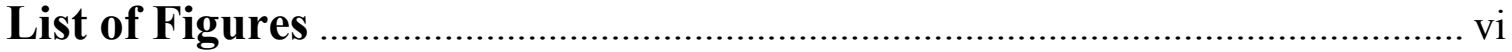

\section{Introduction}

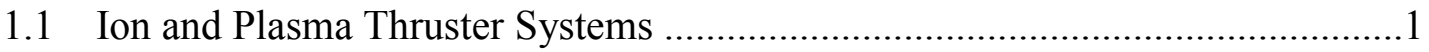

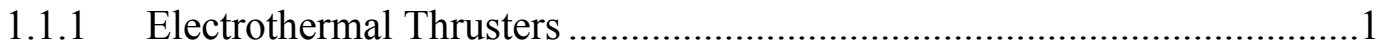

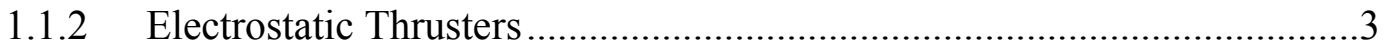

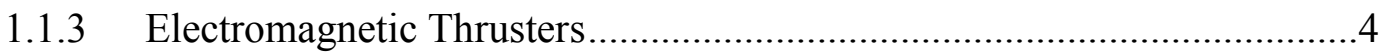

1.2 Applications of Electric Propulsion ................................................................

1.3 Piezoelectric Transformer Plasma Source Micro-Thruster ..................................10

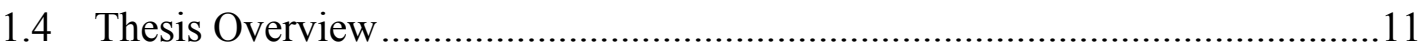

\section{PTPS-MT Operation}

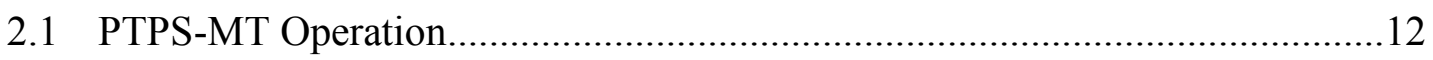

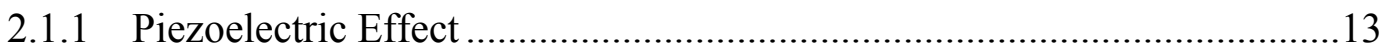

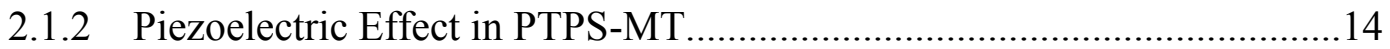

2.1.3 Charged Particle Acceleration ...................................................................

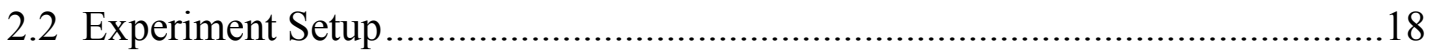

2.2.1 Driving the PTPS-MT ......................................................................

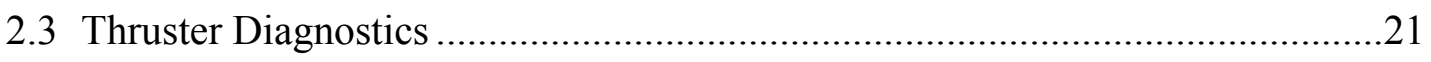

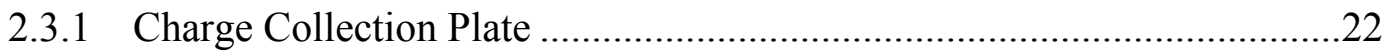

2.3.2 Faraday Cup/ Retarding Potential Analyzer ...............................................23

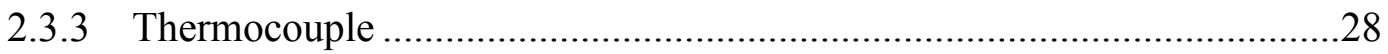

\section{Simulations}

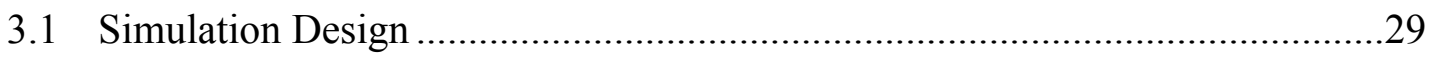

3.1.1 Dielectric Loss Tangent .....................................................................

3.1.2 Mechanical (Structural) Loss Factor........................................................31 


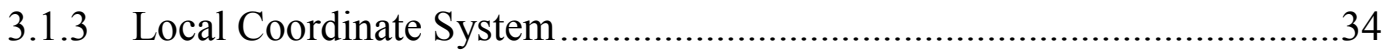

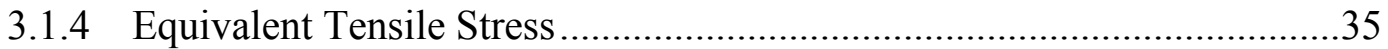

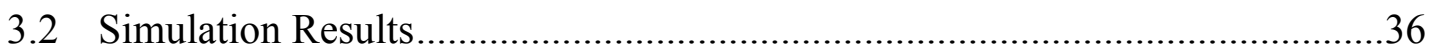

3.2.1 Effect of Aperture Diameter and Offset on Transformation Ratio ............37

3.2.2 Frequency Effect on Transformation Ratio ...........................................41

3.2.3 Effect of Ground Electrode Angular Offset on Transformation Ratio ......43

3.2.4 Effect of Increasing Crystal Thickness ..............................................48

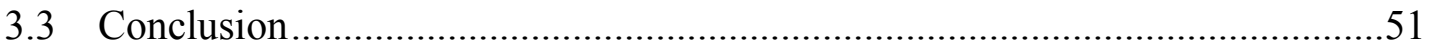

\section{PTPS-MT Thruster Analysis}

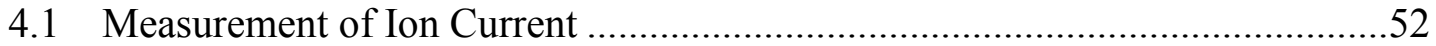

4.1.1 Effect of Aperture Diameter on Collected Current ..................................52

4.1.2 Effect of Driving Voltage on Collected Current.......................................56

4.1.3 Effect of Extraction Voltage on Collected Current...................................60

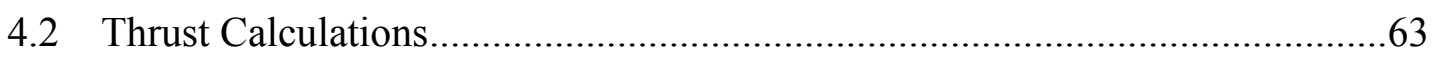

4.2.1 Mass Flow Utilization.....................................................................63

4.2.2 Estimation of Neutral Flow ....................................................................66

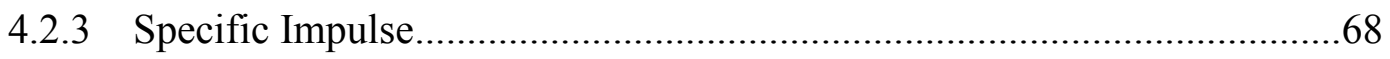

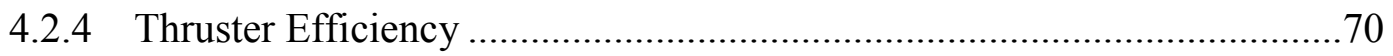

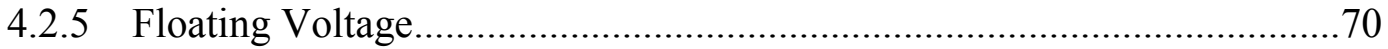

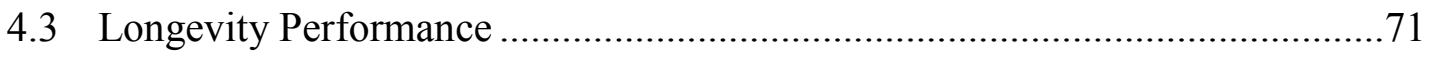

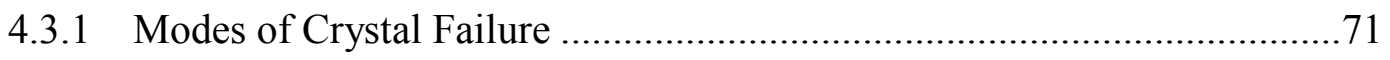

4.3.2 Extended Duration Operation ................................................................ 79

\section{Summary}

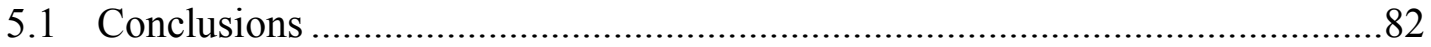

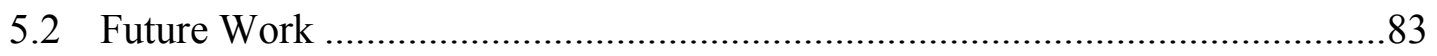

\section{Appendices}

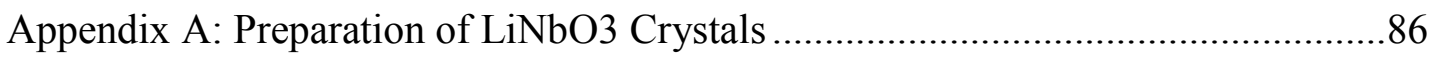

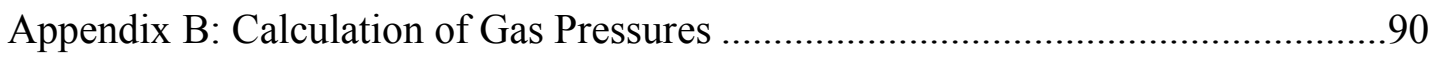

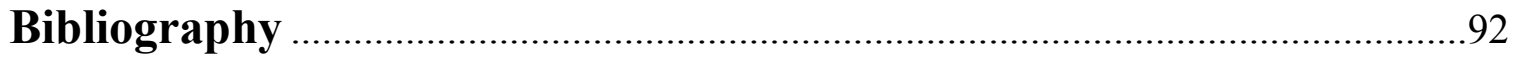




\section{LIST OF TABLES}

Table 1.1: Estimated velocity increments for select missions .....................................8

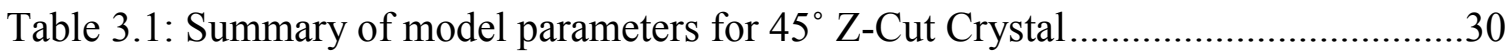

Table 3.2: Voltage transformation sensitivity to shift in frequency ...............................43

Table 3.3: Final crystal design parameters ..............................................................51

Table 4.1: Paschen curve constants for argon and steel............................................59

Table 4.2: Thermal velocities of PTPS-MT gasses ..................................................67

Table 4.3: Selected efficiencies for argon at typical input powers ................................70

Table 4.4: Comparison of transformer core properties ............................................... 74

Table 4.5: Radial change in z-cut lithium niobate based on temperature change.............78 


\section{Chapter 1}

\section{LIST OF FIGURES}

Figure 1.1: Propellant gas is introduced to combustion chamber where a resistance element, $\mathrm{R}$, is used to electrically heat the gas. This results in thermal expansion through a nozzle to produce thrust

Figure 1.2: Produced ions are accelerated through a negatively biased grid to produce thrust. To prevent a space charge from forming, a beam neutralizer must be employed to balance the loss of ions

Figure 1.3: A high electrical current is driven from the anode to the central cathode creating a strong self-induced magnetic field. The resulting interaction of the electric current and magnetic field produces a body force to accelerate a plasma..... .5

Figure 1.4: Comparison of electric propulsion systems 10

\section{Chapter 2}

Figure 2.1: Resonant mechanical strain in radial direction is coupled into strain induced from applied voltage to produce a large voltage on the surface of the disc capable of forming plasma

Figure 2.2: Calculation of optimal electromechanical coupling for lithium niobate based on rotation angle of polarization axis

Figure 2.2: (a) Positive half-cycle applied to driving electrode resulting in perpendicular electric field lines to accelerate ions away from thruster (b) RF signal reverses polarity, changing electric field lines and ejecting electrons .17

Figure 2.3: Estimation of plasma cone half-angle 18

Figure 2.4: Operational setup of PTPS-MT. 18

Figure 2.5: Charge collecting plate used to measure ion current. (1) Switch used to change testing configuration to ground or with a negative DC bias

Figure 2.6: Retarding potential analyzer (1) ground grid, (2) bias grid, (3) secondary suppression grid

Figure 2.7: Ion trajectory path in biased gridded electric field .25

Figure 2.8: Solid angle for a disc source and a detector with a circular aperture [46] ......26

Figure 2.9: Calculation of ion transit time using Faraday cup output 


\section{Chapter 3}

Figure 3.1: (a) 3D model with ground electrode applied (blue) and driving electrode (red). (b) Definition of parameters used in simulations

Figure 3.2: Comparison of piezoelectric holders used in experimentation. (a) Early holder using screw tensioning, (b) Final holder using spring pressure, (c) Early holder disassembled, (d) Spring holder disassembled

Figure 3.3: (a) Plot showing adjustment of mechanical quality through tensioning of electrodes holding piezoelectric disc. (b) Impedance plot of spring tensioned holder requiring no adjustment

Figure 3.4 (1) Standard crystal model with polarization direction in global $\mathrm{Z}$ axis direction (2) Rotated polarization of crystal used in experiments

Figure 3.5 Cross sectional plot of piezoelectric disc showing displacement corresponding to the rotated local axis (global axis shown for reference). Blue slant is due to polarization angle .35

Figure 3.6: Effect of aperture offset on transformation ratio for a $10 \mathrm{~mm}$ diameter crystal. Ground plate aperture diameter of $2.5 \mathrm{~mm}$ used for all locations .38

Figure 3.7: Effect of aperture offset on transformation ratio for a $20 \mathrm{~mm}$ diameter crystal. Ground plate aperture diameter of $5 \mathrm{~mm}$ used for all locations .39

Figure 3.8: Displacement of piezoelectric disc corresponding to ground aperture location of greatest output. Darker red corresponds to greater displacement..... .40

Figure 3.9: Effect of aperture diameter on voltage transformation for $10 \times 2 \mathrm{~mm}$ disc ....41

Figure 3.10: Measured impedance plot showing resonance and anti-resonance frequencies of a piezoelectric disc.

Figure 3.11: Rotated local coordinate system showing cross section of crystal polarization relative to ground aperture .43

Figure 3.12: Effect of ground plate aperture rotation across surface of disc $(20 \mathrm{x} 4 \mathrm{~mm}$

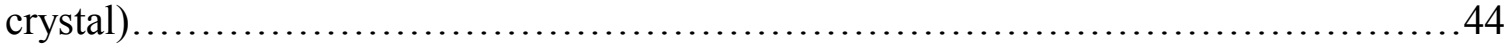

Figure 3.13: Effect of ground plate aperture rotation across surface of disc $(10 \times 2 \mathrm{~mm}$

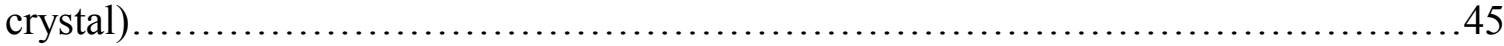

Figure 3.14: Effect of rotating aperture off polarization axis with single resonant frequency of $385 \mathrm{kHz}$. (a) $130^{\circ}$, (b) $100^{\circ}$, (c) $80^{\circ}$, (d) $70^{\circ}$, (e) $250^{\circ}$, (f) $255^{\circ}$, (g) $260^{\circ}$, (h) $280^{\circ}$. .46

Figure 3.15: Rotational plot comparing displacement, potential and stress .47 
Figure 3.16: (a) Von Mises stress plot showing highest stress in center of disc. (b) Crystal failure with fracture through center of disk .48

Figure 3.17: Stress and voltage transformation corresponding to increasing crystal thickness for a $10 \mathrm{~mm}$ diameter crystal and $100 \mathrm{~V}$ applied voltage ................................50

Figure 3.18: Stress and voltage transformation corresponding to increasing crystal thickness for a $20 \mathrm{~mm}$ diameter crystal and $100 \mathrm{~V}$ applied voltage .50

\section{Chapter 4}

Figure 4.1: Physical model of PTPS-MT with containment aperture used to limit neutral flow losses to vacuum..... .53

Figure 4.2: Collected emission current for hydrogen propellant. Use of the $0.9 \mathrm{~mm}$ aperture not included due to inability to form plasma at available flow rates .55

Figure 4.3: Collected ion current for nitrogen propellant at three selected aperture diameters .55

Figure 4.4: Collected ion current for argon propellant at three selected aperture diameters. .56

Figure 4.5: Collected ion current for hydrogen with four increasing applied voltages. Increasing plasma density occurs with an increase in driving voltage .57

Figure 4.6: Collected ion current for nitrogen with four increasing applied voltages .......58

Figure 4.7: Collected ion current for argon with four increasing applied voltages. Paschen curve included (in dark blue) to show minimum breakdown voltage for plasma as function of mass flow rate. .58

Figure 4.8: Collected argon current with three applied extraction voltages. Three effects are noted (a) high electron mobility resulting in collection of negative charge, (b) ion extraction, (c) high plasma density extraction 61

Figure 4.9: Collected nitrogen current with three applied extraction voltages 61

Figure 4.10: Collected hydrogen current with three applied extraction voltages

Figure 4.11: Ion velocities for argon under 50 and $1140 \mathrm{~V}$ applied accelerating voltage .65 Figure 4.12: Maximum obtained ion component of thrust for each propellant. $1190 \mathrm{~V}$ driving voltage and $1140 \mathrm{~V}$ extraction used for all three gasses. Graph endpoints correspond to maximum flow rate for MFC with selected gas. .66

Figure 4.13: Left axis shows ion thrust for argon, while the straight line and right axis represents neutral thrust .68

Figure 4.14: Specific impulse of PTPS-MT for three selected gasses. .69 
Figure 4.15: Floating voltage of thruster assembly with argon gas and $0 \mathrm{~V}$ extraction ....71

Figure 4.16: (a) Early transformer operated at 35V (80\% Amplification), (b) same transformer at $90 \%$ amplification, (c) same transformer at 100\% amplification, (d) new transformer design operated at 100\% amplification .73

Figure 4.17: (a) location of highest stress. (b) resulting crystal fracture profile .75

Figure 4.18: Photo and SEM of crystal after prolonged operation showing four regions of interest: (1) electrode covered by ground aperture, (2) painted electrode exposed to plasma chamber, (3) burn ring from location of triple point/ plasma formation, (4) polished center .76

Figure 4.19: Region 3 of crystal showing significant ablation of surface material from plasma discharge at location of triple-point. .77

Figure 4.20: Region 4 of crystal. Plasma etching created smooth clear surface on crystal which was opaque and unpolished when new. Presence of surface contaminants, most likely from electrode or silver paint, shown as specks .77

Figure 4.21: Stress profile corresponding to a $0.2 \%$ displacement along radial axis. The maximum stress exceeds the fracture stress for lithium niobate.

Figure 4.22: Performance of thruster during four two hour testing cycles. Output normalized to peak current of $2 \mu \mathrm{A}$

Figure 4.23: Recovery time of thruster to return to initial current output at beginning of two hour run. Each recovery period conducted immediately following 2 hour run with thruster and gas off except for to obtain data point .80

Figure 4.24: Measurement of thruster performance over a six day continuous operation. All operating conditions matched the two hour tests . .81

\section{Chapter 5}

Figure 5.1: Recommended thruster design for any future approaches to the PTPS-MT...87 


\section{Chapter 1}

\section{Introduction}

The science and technology of electric propulsion is an important field of research that aims to overcome a number of limitations found in conventional chemical propulsion systems [1]. Traditional methods of acceleration are provided by chemical propellants which use the thermal expansion of combustion gasses to create thrust [2]. Peak exhaust velocities depend on achieving high exhaust temperatures, which are limited by the type of fuel and the material properties of the thruster nozzle [3],[4]. Conventional systems also provide a low specific impulse, a measure of rocket efficiency relating thrust to propellant usage [5],[6]. Electric propulsion systems may overcome many limitations of conventional propulsion mechanisms by employing techniques to obtain higher exhaust velocities while reducing propellant utilization and vehicle launch weight [4]. Of these mechanisms, three common approaches are often referenced: 1) electrothermal, 2) electrostatic, and 3) electromagnetic acceleration [1].

\subsection{Ion and Plasma Thruster Systems}

\subsubsection{Electrothermal Thrusters}

Of the many electric propulsion designs, electrothermal thrusters most closely resemble the design and operation of a conventional chemical rocket [7]. In an electrothermal system, a propellant gas is heated electrically and then expanded through a nozzle to convert thermal energy to kinetic energy which is described by Equation 1.1 [4], [7]. 


$$
\frac{1}{2} u_{e}^{2}=\frac{1}{2} u_{c}^{2}+c_{p}\left(T_{c}-T_{e}\right)
$$

Here $u_{e}$ is the exhaust speed, $u_{c}$ is the chamber flow velocity, $c_{p}$ is the specific-heat of the combustion gas, and $T_{c}$ and $T_{e}$ are the chamber and exhaust tempertures. From the above equation, it becomes clear that a high chamber temperature is desired to achieve a high exhaust velocity.

Methods of heating include solid resistance elements and arc discharge [4],[7],[8]. Figure 1.1 details the basic concept of operation where an electric resistor element is used to heat and expand a propellant through the rocket nozzle. As with conventional rockets, chamber temperature is limited by the ability of the wall material to withstand failure through overheating [6],[8]. This often limits the application of electrothermal thrusters. With the added weight and design costs of a separate power supply needed to power such a system, applicability over conventional thrusters relies heavily on achieving high propellant and operation efficiencies [4]. These systems are often found on satellites and used for orbital maintenance because of their improved propellant utilization efficiency [4],[9].

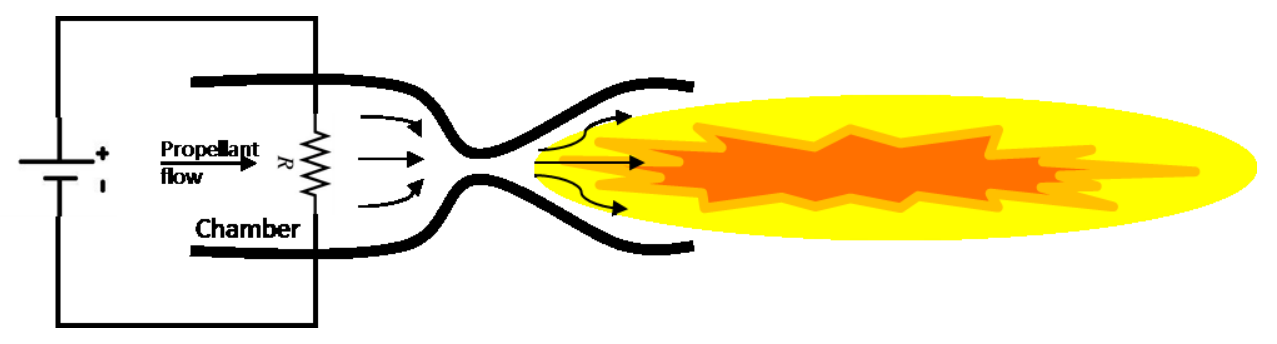

Figure 1.1: Propellant gas is introduced to combustion chamber where a resistance element, $\mathrm{R}$, is used to electrically heat the gas. This results in thermal expansion through a nozzle to produce thrust. 


\subsubsection{Electrostatic Acceleration}

Unlike electrothermal applications, where the maximum attainable velocity is largely dependent on thermal limitations, electrostatic acceleration utilizes the Coulomb force to achieve high exhaust velocities. First, a source of positively charged ions must be produced. This can be done in a number of different ways, with the most common approach using high energy electrons to induce ionization in neutral molecules according to the reaction:

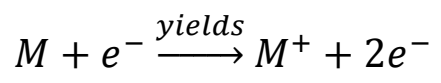

By applying a large potential at the accelerating grid, the magnitude of the force, $F$, applied on the ions results in large exhaust velocities, $V_{e}$, according to Equation 1.2:

$$
V_{e}=\int_{t_{0}}^{t_{f}} F m_{i} d t \quad[\mathrm{~m} / \mathrm{s}]
$$

Here, $m_{i}$, is the mass of the ion created from the chosen propellant gas.

Therefore, the maximum thrust attainable in electrostatic systems depends on the mass of the accelerated ion, the accelerating potential and the total plasma flux that can be generated by the ion source [5]. Electrostatic acceleration has been used in many NASA space programs including NASA's Evolutionary Xenon Thruster (NEXT) [10], [11]. The efficiency of electrostatic acceleration comes at the cost of intricate designs requiring beam neutralization, beam shaping and large power supplies for both ionization and accelerating potentials [4], [7], [12].

Operation of most electrostatic thrusters follow the design in Figure 1.2. Electron bombardment or arc discharge are often used to ionize the source gas [13], [14]. Xenon is the most commonly used propellant because of the large ion mass [15]. Once ions are 
generated, an electric grid is used to draw out and accelerate the positively charged ions to generate thrust. To prevent the spacecraft from developing an electric charge due to the loss of ions, a beam neutralizer is used, which consists of an electron emitting cathode to provide electrons, keeping the spacecraft in a neutral state and preventing loss of thrust through the return of ions should the spacecraft develop a negative charge [16].

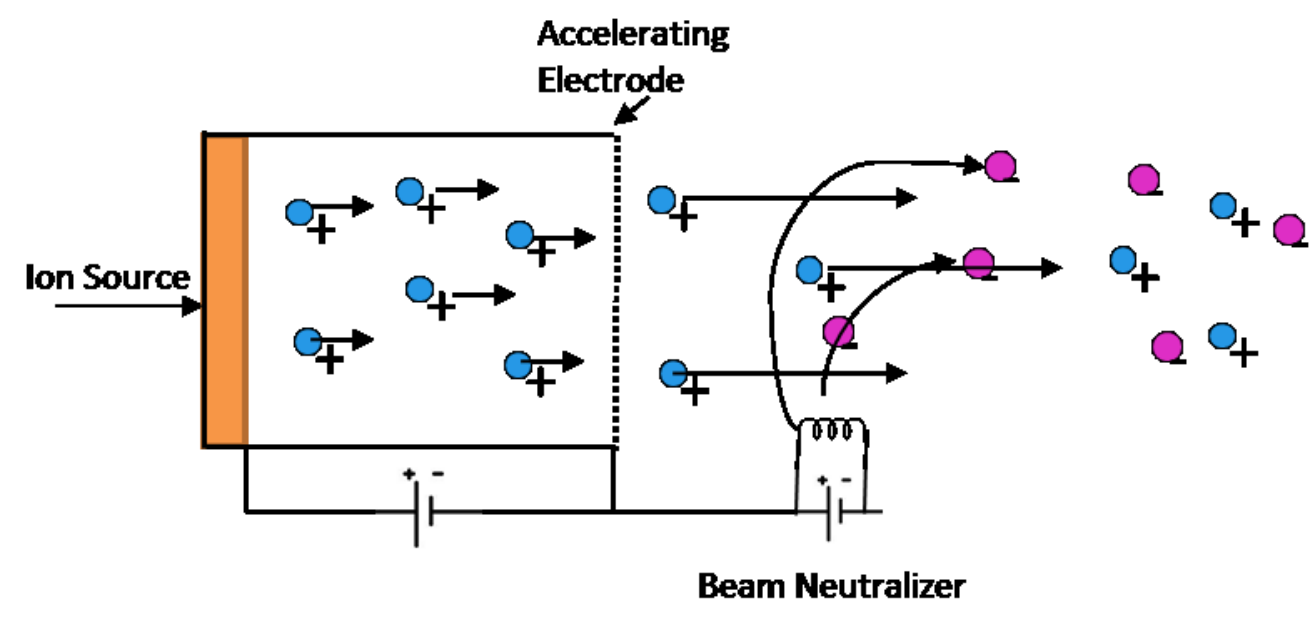

Figure 1.2: Produced ions are accelerated through a negatively biased grid to produce thrust. To prevent a space charge from forming, a beam neutralizer must be employed to balance the loss of ions.

\subsubsection{Electromagnetic Acceleration}

Electromagnetic acceleration uses the Lorentz force though the interaction of currents driven through a gas with magnetic fields to accelerate ions [17],[18]. According to Lorentz's Law, any charged particle traveling in an electric and magnetic field will experience a force, as described by Equation 1.3:

$$
F=q[E+(\boldsymbol{v} \times \boldsymbol{B})]
$$

where $q$ is the charge density of the gas, $E$ is the electric field, $v$ is the gas velocity and $B$ is the magnetic field. 
By driving a high current through the gas parallel to the E field, an accelerating body force is applied to the ions according to:

$$
F_{b}=j \times B
$$

where $j=q v$ is the current density and $\boldsymbol{F}_{\boldsymbol{b}}$ is the body force accelerating the ions.

Similar to an electrostatic thruster, a propellant gas is first ionized. The positively charged ions are then accelerated by the magnetic field, which can be externally applied or induced by the current flowing through the plasma [18]. The resulting interaction between the current and magnetic fields is an applied thrust, shown in Figure 1.3.

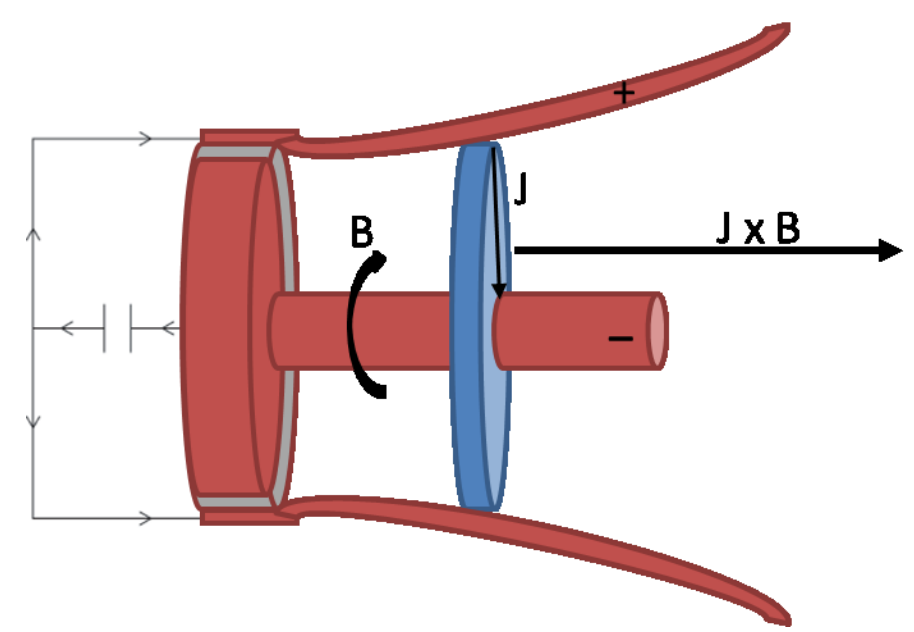

Figure 1.3: A high electrical current is driven from the anode to the central cathode creating a strong self-induced magnetic field. The resulting interaction of the electric current and magnetic field produces a body force to accelerate a plasma.

In comparison to the other two mechanisms, electromagnetic acceleration is considered the most complex and difficult to implement. The main drawback is the power supply requirement to produce current in excess of $100 \mathrm{~A} / \mathrm{m}^{2}$, which is necessary to generate a large electric and magnetic field used for acceleration [4]. Degradation of 
thruster components at these high currents significantly limits the operational lifetime and is considered one of the main limitations of electromagnetic acceleration [19]. However, the benefit of this technique is the highest experimental exhaust velocities and total thrust for electric propulsion systems to date, with developed systems already demonstrating velocities of over $100,000 \mathrm{~m} / \mathrm{s}$ and $100 \mathrm{~N}$ of thrust [20].

The Variable Specific Impulse Magnetoplasma Rocket (VASIMR) is one system that uses the electromagnetic method of acceleration [21]. Advances in low weight, high voltage and high efficiency power supplies are needed before this form of electric acceleration becomes viable.

\subsection{Application of Electric Propulsion}

While electric propulsion offers many advantages over conventional rockets, application is limited to low drag, low gravity environments, such as high earth orbit or a space environment, due to the low thrust afforded by these systems. To highlight applications where electric propulsion is a desirable choice, a comparison of thrust, specific-impulse, and exhaust velocity to a conventional rocket is needed. To do this, consider a mission to Mars, consisting of leaving the Earth's surface, accelerating from orbit, decelerating at the destination, and the same on the return to Earth. The fuel requirements and mission length quickly become prohibitive on a conventional, chemical-based rocket system [4]. While such a method is possible for a "small" distance to Mars and back, deep space missions are unfeasible. To illustrate this, consider the basic flight of a rocket in a gravitational field:

$$
m \dot{\mathbf{v}}=\dot{m} \mathbf{u}_{\mathbf{e}}+\boldsymbol{F}_{\boldsymbol{g}}
$$


where $\dot{\mathbf{v}}$ is the acceleration vector of the rocket, $\dot{m}$ is the rate of change of rocket mass through propellant loss, $\mathbf{u}_{\mathrm{e}}$ is the exhaust velocity relative to the rocket and $\boldsymbol{F}_{\boldsymbol{g}}$ is the local gravitational force. The total thrust over the full use of the propellant is its total impulse, I, or:

$$
I=\int_{t_{0}}^{t_{f}} \dot{m} \boldsymbol{u}_{e} d t
$$

Assuming that an electrical propulsion device would operate outside a local gravitational force (a requirement for the low magnitude of thrust under consideration), the motion equation integrates to:

$$
\Delta \mathrm{v}=\mathbf{u}_{\mathbf{e}} \ln \frac{m_{0}}{m_{f}}
$$

Here, $\Delta \mathrm{v}$ is the velocity increment achieved over the full use of propellant, $m_{0}$ is the initial mass of the rocket, including all fuel, and $m_{f}$ is the final mass of the rocket after fuel has been used. Based on Equation 1.8, a high exhaust velocity is needed to reach a desired velocity increment [4].

Many space missions are characterized by the velocity increment. For example, maintaining a satellite orbit over many years would have a high $\Delta \mathrm{v}$, as would a mission to a distant planet and back. Many of these missions have already been analyzed separately. A summary of select missions are given in Table 1.1. With most conventional chemical rockets, attainable exhaust velocities average around 4,500 m/s [22]. Compared to chemical systems, average ion thrusters often achieve exhaust speeds upwards of $90,000 \mathrm{~m} / \mathrm{s}$ with experimental systems reaching beyond 240,000 m/s [6], [14]. From Table 1.1, it is clear that missions outside our solar system will require much higher 
exhaust velocity increments than chemical systems can provide. This is one area where electric propulsion is attractive for space applications.

Table1.1: Estimated velocity increments for select missions [4], [23]

\section{Mission}

Escape from earth surface (impulsive)

Escape from 300-mile orbit (impulsive)

Earth orbit to Mars orbit and return

Earth surface to Mars surface and return

Earth orbit to Jupiter orbit and return

Earth orbit to Saturn orbit and return

\section{$\Delta \mathrm{V}, \mathrm{m} / \mathrm{sec}$}

$1.12 \times 10^{4}$

$3.15 \times 10^{3}$

$1.4 \times 10^{4}$

$3.4 \times 10^{4}$

$6.4 \times 10^{4}$

$1.1 \times 10^{5}$

A simple comparison of exhaust velocity increments are not enough to fully compare conventional to electric propulsion systems. While an electrical system may provide much higher velocities, it does so at the cost of thrust. Most electrical systems provide usable thrust on a scale of $\mathrm{mN}$, with some advanced systems providing up to $100 \mathrm{~N}$ of thrust [16]. For comparison, the NASA space shuttle provides over $25 \times 10^{6} \mathrm{~N}$ of thrust at launch [24]. To highlight the importance of this parameter, a quick calculation of a small one-ton rocket requires a minimum of 9,000 N of thrust to overcome Earth's gravitational pull. With current technologies, application of electric propulsion in the vicinity of a gravitational field is not feasible.

Velocity increments and thrust values provide concrete parameters for comparison of each system, but still do little to compare a system's overall viability. For example, a low thrust provided over a long time can provide the same final velocity as a 
high thrust over a short period of time. Technically, both methods could work to reach a far-off destination. A comparison of the propellant use to thrust, or specific impulse, provides the clearest overall picture. A high specific impulse means a lower use of propellant is needed to provide an equivalent amount of thrust. On long duration missions, efficiency becomes very important. A highly efficient system has less need for fuel, meaning more of the spacecraft's mass could be devoted to other areas, such as instrumentation.

A summary of current electrical propulsion systems is provided in Figure 1.4. These will be used as a comparison for PTPS-MT results. For reference, a typical chemical rocket has an average specific impulse of $300 \mathrm{~s}$ [16].

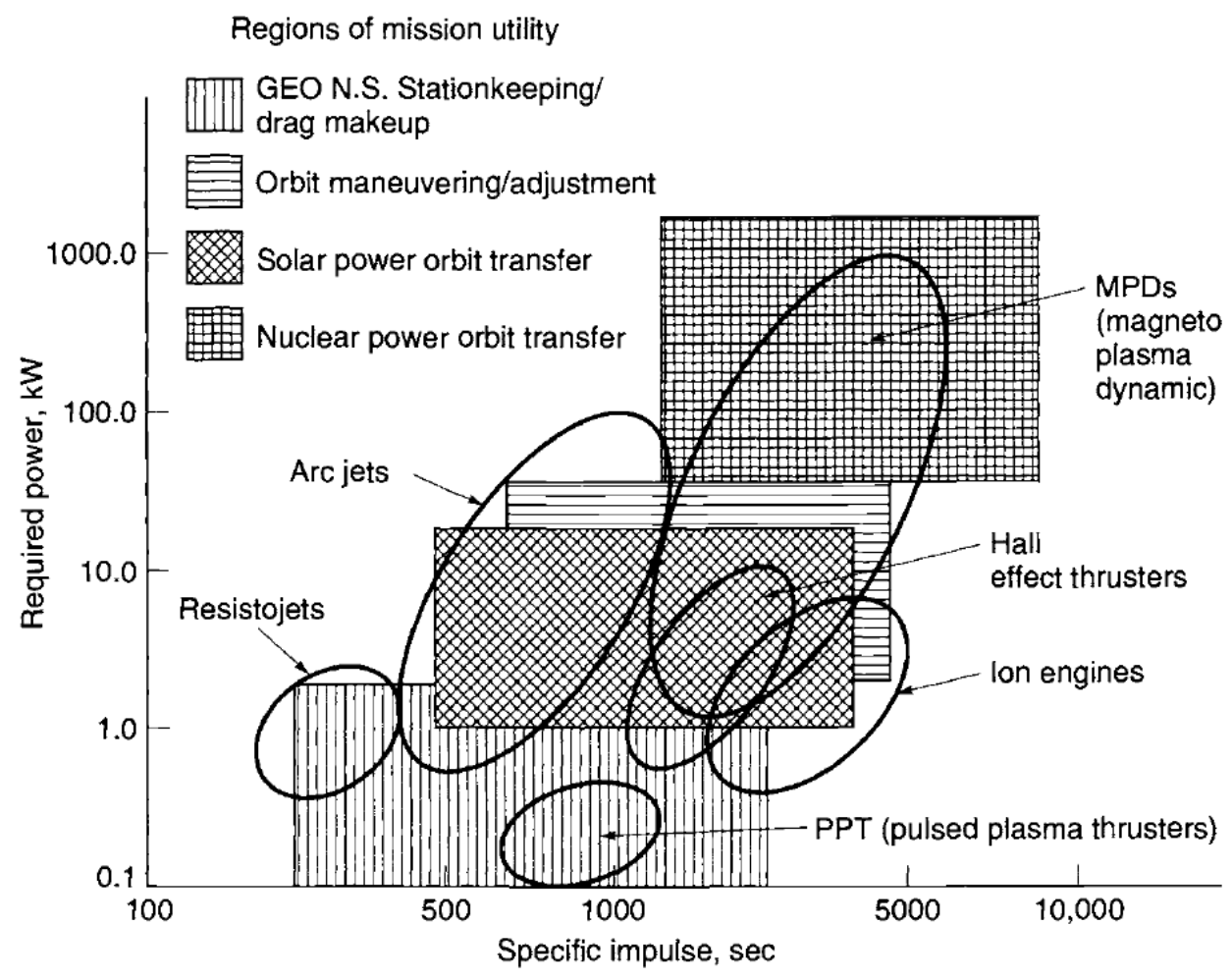

Figure 1.4: Comparison of electric propulsion systems [39] 


\subsection{Piezoelectric Transformer Plasma Source Micro-Thruster}

The piezoelectric transformer plasma source micro-thruster (PTPS-MT) is a unique system that draws on previously described electric propulsion methods to form a low power, small form-factor thruster. The PTPS-MT uses a $10 \mathrm{~mm}$-diameter x $2 \mathrm{~mm}$ thick lithium niobate crystal to step-up an input voltage to ionize a propellant gas. By operating at the resonant frequency of the crystal, a high step-up voltage transformation can be achieved with a $10 \mathrm{~W}$ power supply [25]. Typical electric propulsion methods require very large or multiple high-voltage power supplies to achieve similar operating conditions [4], [10], [12].

In contrast to the other electrical thrusters, the PTPS-MT is a self-neutralizing system, using an RF signal to accelerate ions or electrons during each half cycle. This negates the need for complex beam neutralization procedures needed to prevent a spacecraft from developing a large net charge. Without a neutralization system, the charging of the spacecraft could potentially effect equipment, reduce the thrust or possibly result in a complete system failure. The PTPS-MT can also be operated with an electrostatic accelerator, similar to an ion thruster, with minimal configuration changes. A complete description of the design and operation of the PTPS-MT will be presented in Chapter 2. 


\subsection{Thesis Overview}

Chapter 2 describes the basic setup used in the operation of the PTPS-MT, diagnostic systems, and methods for analysis of data. Chapter 3 describes experimental simulations used to determine operating parameters and validation of assumptions used in thruster operation. Results from the modeling were used to improve the PTPS-MT design. Chapter 4 provides results on the measured ion current and calculated thrust provided by the PTPS-MT before concluding with discovered modes of failure and lifecycle analysis. Chapter 5 concludes the thesis and presents recommendations for future work. 


\section{Chapter 2}

\section{Piezoelectric Transformer Plasma Source Micro-Thruster (PTPS-MT) Operation}

This chapter details the operation and theory of the PTPS-MT design, equipment needed for operation and the diagnostic tools used in the characterization of the PTPSMT. It will also cover the selection of basic operational parameters used in testing.

\subsection{Piezoelectric Transformer Plasma Source Micro-Thruster}

The PTPS-MT uses a single crystal lithium niobate disc for ionization and acceleration of ions and electrons. Lithium niobate exhibits properties of being a piezoelectrictric, ferroelectric and pyroelectric material [26]. While all three properties are capable of producing ions and developing accelerating potentials, the PTPS-MT is operated under conditions where only the piezoelectric effect is applicable. To reach conditions where the ferroelectric properties of lithium niobate exist requires the electric field applied through the crystal to exceed $210 \mathrm{kV} / \mathrm{cm}$ [27]. The maximum electric field used in the PTPS-MT never exceeds $10 \mathrm{kV} / \mathrm{cm}$, therefore ferroelectric effects are not considered as a mechanism for ionization and acceleration. Similarly, for lithium niobate to exhibit the pyroelectric effect, an increase or decrease in the temperature must occur. If the temperature is held constant over a period of time, the pyroelectric polarization gradually disappears due to leakage current [26]. Operation of the PTPS-MT has shown a rise in temperature in the most extreme operating regimes done during experimental testing. For all but a few temperature specific experiments, operation of the PTPS-MT 
was done under conditions of negligible temperature increase and was held steady over the duration of testing. Therefore, the pyroelectric effect is not considered in this analysis.

\subsubsection{Piezoelectric Effect}

The piezoelectric effect is the linear electromechanical relationship between the mechanical and electrical states of crystalline materials [20]. It is a reversible process in which crystals that experience the direct piezoelectric effect also exhibit the converse piezoelectric effect. The direct effect is the internal generation of electric charge as a result of mechanical stress induced in the crystal, while the converse effect is the mechanical displacement of the crystal as a result of induced charge [20]

While many systems rely on either the direct or converse piezoelectric effect to perform an action, the piezoelectric transformer uses both effects in a single device $[29,30]$. The converse effect is used to convert an applied voltage into a mechanical displacement within the piezoelectric material, while the direct effect is used to convert the mechanical stress into an electric potential at the transformer output. Among the advantages offered by the piezoelectric transformer are significant reductions in mass and volume, resistance to electromagnetic noise, and inherent high-voltage isolation [28]. These properties are ideal for the PTPS-MT, allowing for a lightweight high-voltage power source to ionize and accelerate ions in a single compact system.

Piezoelectricity can be described mathematically by relating the electrical properties of a material, as in Equation 2.1, to the elastic properties shown in Equation 2.2. The two are related by the piezoelectric constant, $e$, given in the coupled equations, 2.3 and 2.4: 


$$
\begin{gathered}
D=\in E \\
S=s T \\
\{T\}=\left[c^{E}\right]\{S\}-[e]^{t}\{E\} \\
\{D\}=[e]\{S\}+\left[\epsilon^{S}\right]\{E\}
\end{gathered}
$$

In Equations 2.1-2.4, $T$ is the stress, $S$ is the strain, $E$ is the electric field, and $D$ is the electric displacement. Parameters $c, \epsilon$, and $e$ represent the stiffness, dielectric and piezoelectric coupling constants, respectively, for a material. The stress-charge form of the constitutive piezoelectric equations is used over the more common strain-charge form due to the requirements of COMSOL [29]. Detailed discussion on the rotation of the polarization axis and its effect are discussed in Chapter 3.

\subsubsection{Piezoelectric Effect in PTPS-MT}

The PTPS-MT was driven with a low voltage through the thickness of the disc along the z-axis at a frequency corresponding to the mechanical resonance in the radial direction according to Figure 2.1:

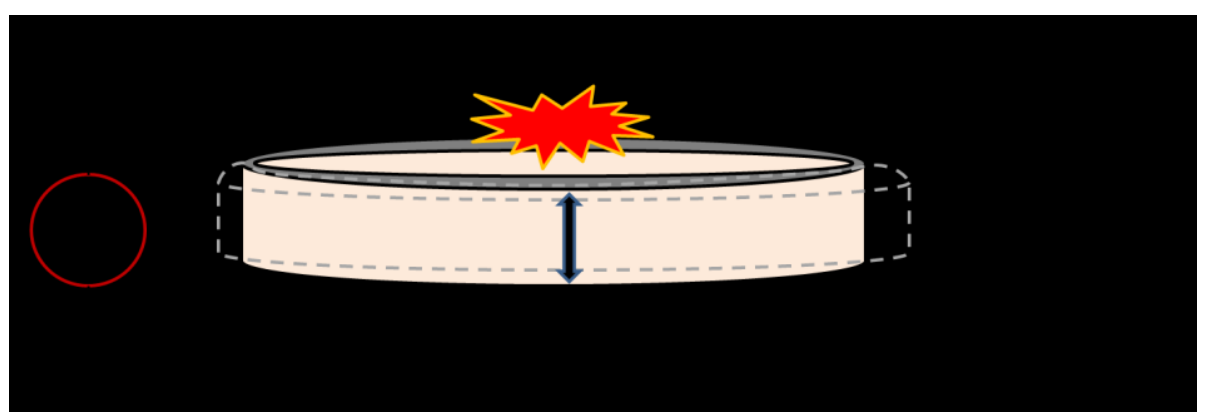

Figure 2.1: Resonant mechanical strain in radial direction is coupled into strain induced from applied voltage to produce a large voltage on the surface of the disc capable of forming plasma. 
The RF voltage produced a strain in the thickness direction that then coupled into the mechanical resonance strain according to properties of the selected piezoelectric material. The effectiveness of this coupling is described by the electromechanical coupling factor, $\mathrm{k}$, and is well documented for lithium niobate. Changes in coupling occur as the polarization axis of the disc is rotated, with the optimum rotation occurring at $135^{0}$ for a rotated y-cut disc $\left(45^{0}\right.$ for a rotated z-cut disc) as seen in Figure 2.2.

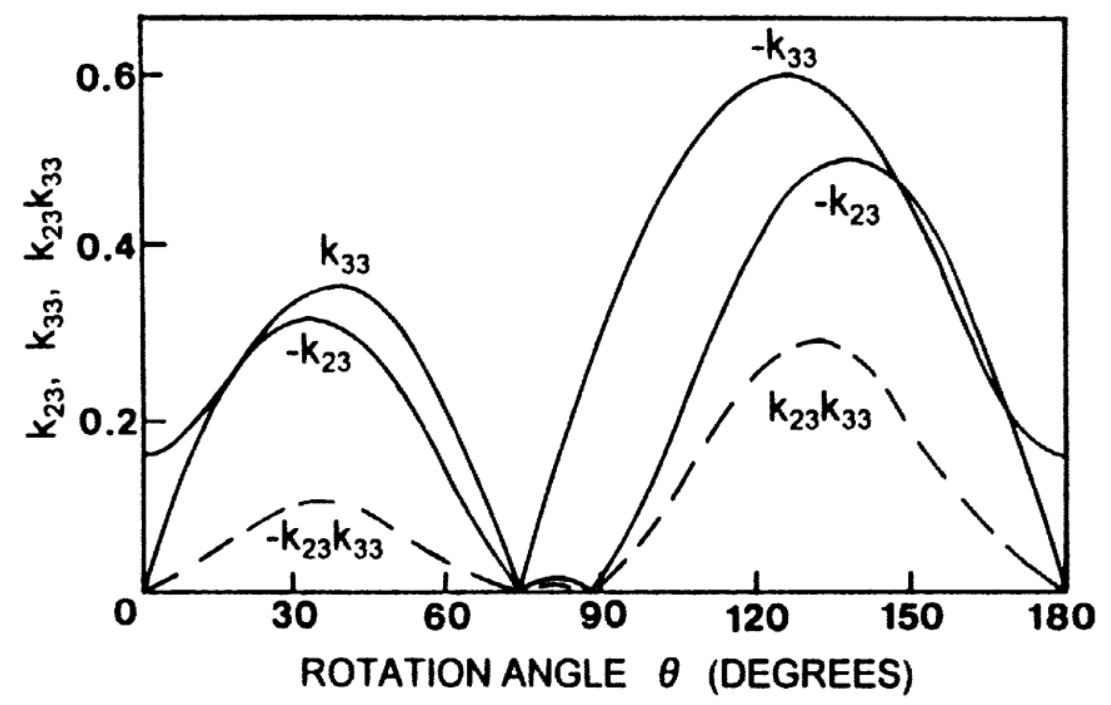

Figure 2.2: Calculation of optimal electromechanical coupling for lithium niobate based on rotation angle of polarization axis [30].

Verification of the coupling factor in laboratory discs could be calculated according to Equation $2.1[30]$ :

$$
k_{p}=\sqrt{\frac{2.51\left(f_{a}-f_{r}\right)}{f_{a}}-\left(\frac{\left(f_{a}-f_{r}\right)}{f_{a}}\right)^{2}}
$$

where $k_{p}$ is the combined electromechanical coupling for a radial resonant disc, $f_{a}$ is the anti-resonant frequency of the crystal, and $f_{r}$ is the resonant frequency of the crystal. 
Calculated coupling coefficients for the lithium niobate crystals ranged between 0.22 0.26. The resulting electromechanical coupling resulted in a high voltage being developed on the surface of the disc. This voltage has a gradient along the surface with simulations showing the highest potential located towards the edge of the disc and approximately 90 degrees off the polarization axis direction. The voltage gradation produces a strong electric field normal to the disc that is capable of ionizing a background gas and providing an accelerating potential. In depth discussion on the gradient and high potential location will be presented in Chapter 3 simulations.

\subsubsection{Charged particle acceleration}

Charged particle acceleration in the PTPS-MT occurs when electric fields generated within the aperture radiate outward from the surface of the thruster to accelerate oppositely charged particles during each half cycle of the RF period. Electrostatic simulations of the thruster show electric field lines perpendicular to the surface of the crystal which rapidly decrease in magnitude as they travel outward. Ions and electrons are estimated to follow a path similar to the field lines during their emission [25]. When a positive voltage is applied to the driving electrode, the field lines point outward from the thruster, accelerating ions as shown in Figure 2.2(a). During the opposite half cycle, the field lines reverse producing a similar effect with free electrons as shown in Figure 2.2(b). 


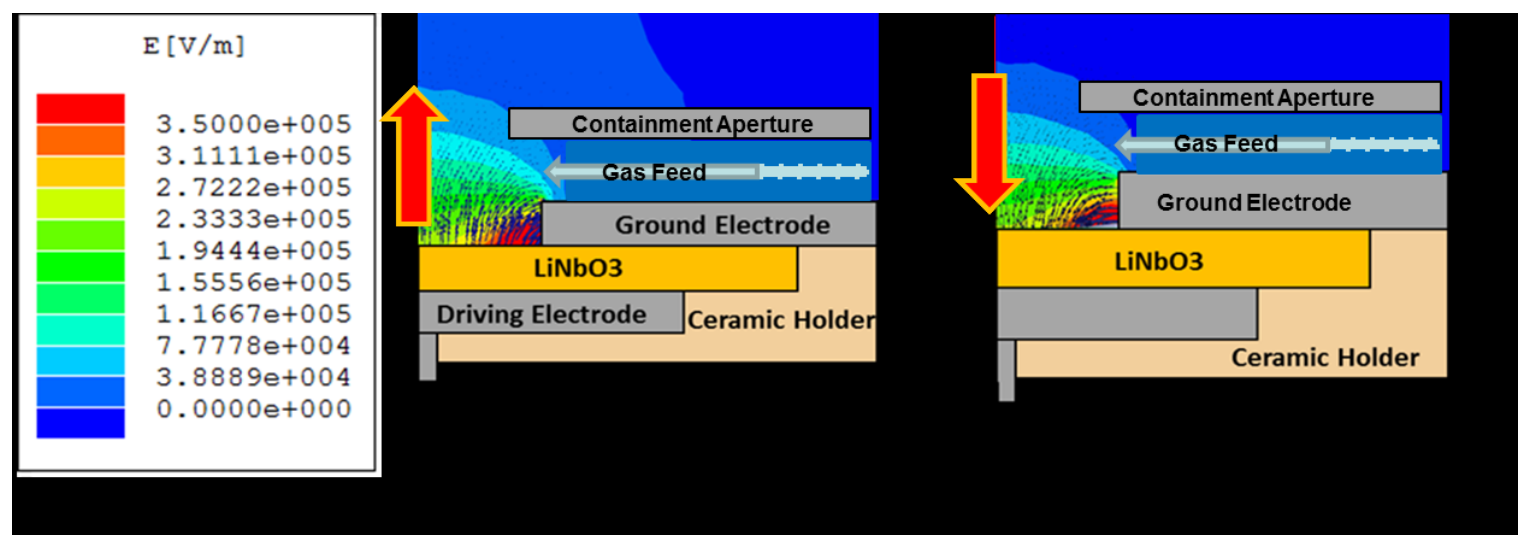

Figure 2.2: (a) Positive half-cycle applied to driving electrode resulting in perpendicular electric field lines to accelerate ions away from thruster (b) RF signal reverses polarity, changing electric field lines and ejecting electrons.

The thrust produced by the accelerating fields can be calculated according to the mass flow rate of the source gas and the velocity of the ejected particle according to:

$$
T=\dot{m}_{i} v_{i}
$$

Where $\mathrm{T}$ is the thrust, $\dot{m}_{i}$ is the ion mass flow rate and $v_{i}$ is the ejected ion velocity. The thrust contributed by the electron discharge is more than seven orders of magnitude smaller than the ion thrust and is neglected for consideration. A correction to account for the beam spread and the loss of thrust associated with ions being ejected at angles off the normal axis. Using the assumption that the PTPS-MT operates as a point source and the beam is uniformly distributed, a correction for thrust due to beam divergence can be found by calculating the cosine of the half angle [31]:

$$
F_{t}=\cos (\theta)
$$

The calculation of the half angle was approximated by photographing a picture of the beam and estimating the angle according to Figure 2.3. The resulting angle was 
determined to be $48^{0}$. This corresponds to a $33 \%$ reduction in the true thrust according to the mass flow analysis.

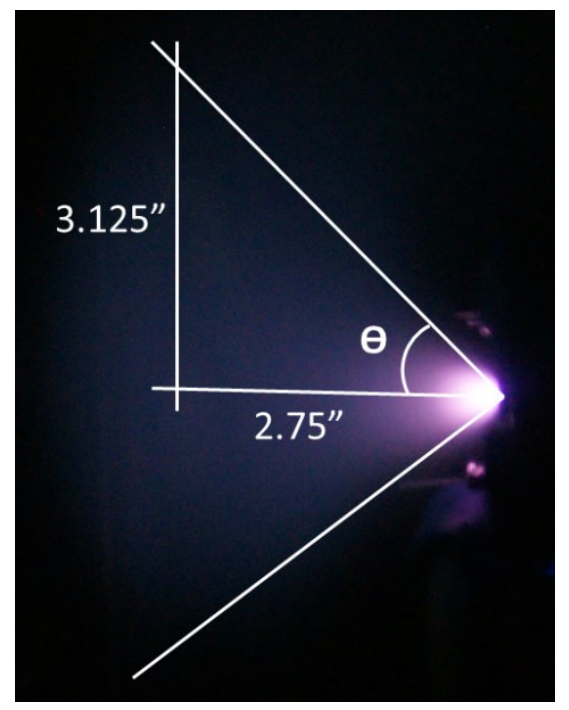

Figure 2.3: Estimation of plasma cone half-angle.

\subsection{Experimental Setup}

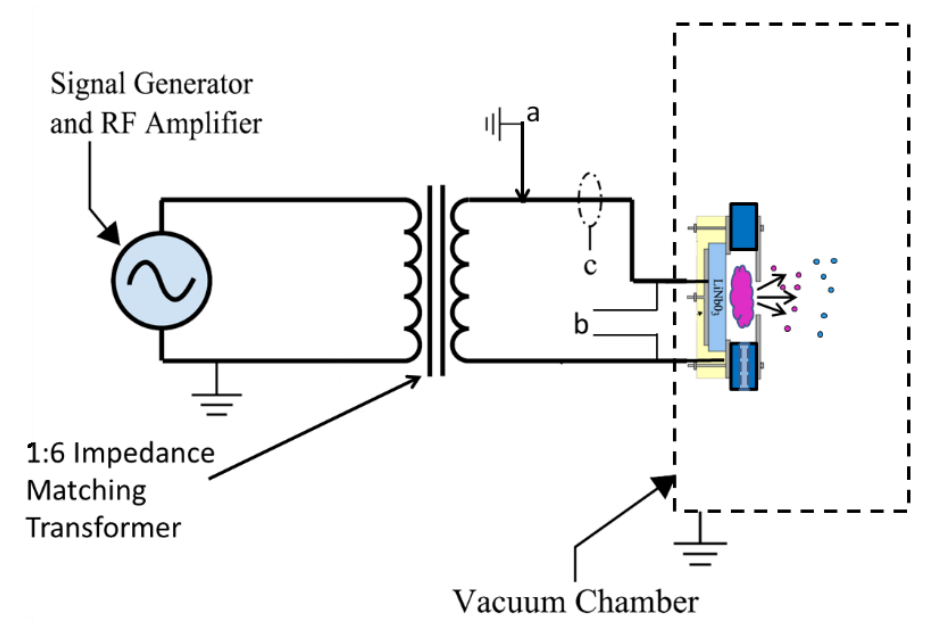

Figure 2.4: Operational setup of PTPS-MT (a) Floating Voltage Probe, (b) Differential Probe, (c) Pearson current monitor 


\subsubsection{Driving the PTPS-MT}

The configuration used to drive the PTPS-MT is shown in Figure 2.4. The thruster was driven with an RF voltage at the crystal resonant frequency of $387 \mathrm{kHz}$. Testing was performed using an RF burst mode of 300 to 50,000 cycles and periods ranging from 3 to $150 \mathrm{~ms}$. The burst cycles and periods were chosen to control the operating time of the thruster and limit the physical heating of the crystal. Typical operation was 300 cycles with a burst period of $40 \mathrm{~ms}$, corresponding to an approximate $2 \%$ duty cycle. This operation resulted in consistent experimental results for ion current over a 45 minute testing period, negating the need to correct for changes in ion output over a test. Higher duty cycles near $100 \%$ allowed for less than 5 minutes of testing over which a $70 \%$ decrease from initial ion current was seen. This rapid change in thruster performance was attributed to the fast temperature rise measured on the crystal surface at high duty cycles and will be discussed in detail in Chapter 4 .

The RF signal was generated by an Agilent 33210A waveform generator. Output from the waveform was input into an Amplifier Research 25A250A amplifier to increase the voltage and power of the driving signal. The output of the amplifier fed into a 1:6 step-up impedance matching transformer to match the $50 \Omega$ amplifier output to the calculated $1800 \Omega$ impedance of the thruster. The minimum power to produce reliable plasma was approximately $10 \mathrm{~W}$ at the transformer output to the crystal. Thruster input voltages were measured at the transformer output using a Tektronix P5100A (100x) voltage probe referenced to the transformer ground to measure the floating voltage. A Tektronix P5200A (500x) differential probe was used to measure the input voltage to the 
thruster when it was operating in a floating state. Thruster input current was measured using a Pearson 2877 (1V/A) current monitor.

Vacuum operation required the addition of a gas as an ionization source. Depending on the experiment, nitrogen, argon or helium gas was introduced to the thruster through a channel located just above the surface of the crystal (see Figure 2.5). An aperture located above the plasma chamber, with a diameter ranging from $.1 \mathrm{~mm}$ to $1.3 \mathrm{~mm}$, permitted a local high pressure of around 100 mTorr to develop in the differentially pumped plasma chamber. The high pressure aided ionization by increasing the collision frequency of gas atoms according to:

$$
v \equiv \frac{1}{\tau}=n_{g} \sigma v[\text { interactions } / \text { second }]
$$

where $v$ is the collision frequency, $\tau$ is the time between interactions, $n_{g}$ is the plasma chamber gas density, $\sigma$ is the ionization cross section and $v$ is the electron velocity [45]. Gas flow was controlled using a Celerity 7300 mass flow controller calibrated to a maximum flow of $30 \mathrm{sccm}$ using nitrogen gas.

Ionization of the background gas could also be increased by improving the ionization cross section, $\sigma_{i z}$, through an increase in the applied voltage according to:

$$
\sigma_{i z}=\pi\left(\frac{e}{4 \pi \epsilon_{0}}\right)^{2} \frac{1}{\varepsilon}\left(\frac{1}{\varepsilon_{i z}}-\frac{1}{\varepsilon}\right)
$$

Where $\sigma_{i z}$ is the ionization cross section, e is the electron charge, and $\varepsilon$ is the applied electric field. The ionization cross section reaches its maximum at $\varepsilon=2 \varepsilon_{i z}$ [32]. Typically, the energy required to ionize an atom, such as argon, is approximately 10$15 \mathrm{eV}$. However, not all of the neutral gas is readily ionized. Often a significant portion of the discharge energy goes into production of excited neutral atoms at the cost of 
ionization. Excited atoms, despite having a higher energy level, are electrically neutral for thruster applications. Therefore, ions in an excited state do not interact with the accelerating fields to produce thrust. The effect of this loss is the requirement that the PTPS-MT be operated at voltages and input powers that are well over 10 times the ionization potential to achieve a good production of ions for thrust.

\subsection{Thruster Diagnostics}

To characterize the performance of the PTPS-MT, a series of diagnostic equipment were required. With optimization of thrust being the final goal of research, parameters having an effect, such as output ion and electron current and crystal temperature, were of interest. Three types of diagnostic equipment were used to gather results: 1) a charge collecting plate to quickly gather information on output current in response to changes in the PTPS-MT, 2) a retarding potential analyzer with Faraday cup to measure ion and electron energies individually, 3) a thermocouple measuring circuit to monitor the temperature of the crystal and relate to thruster performance. 


\subsubsection{Charge Collection Plate}

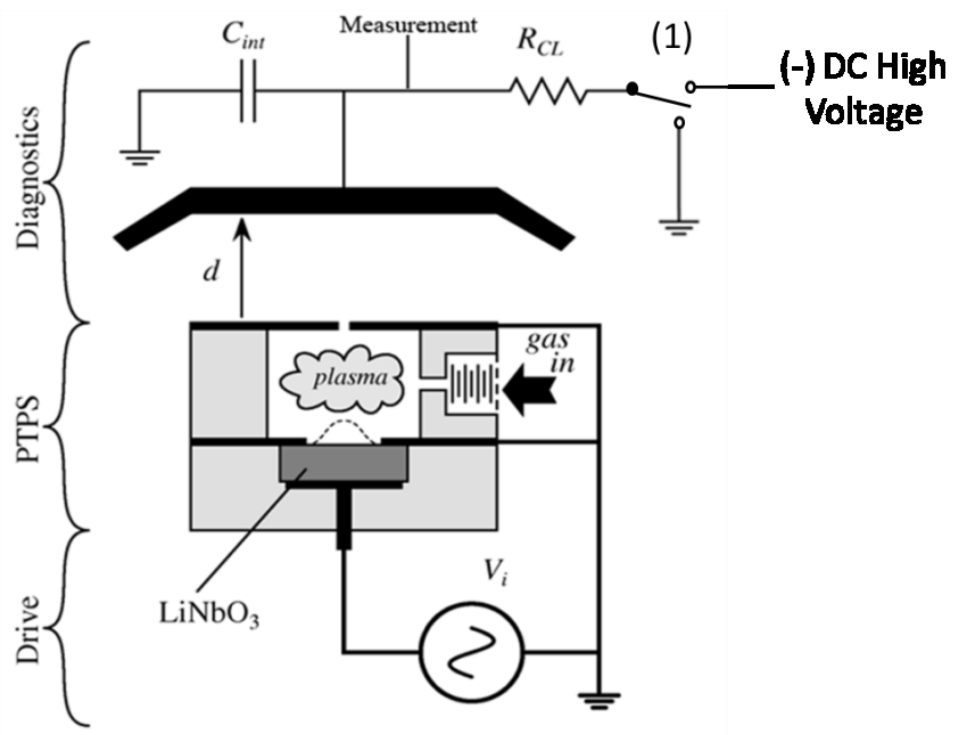

Figure 2.5: Charge collecting plate used to measure ion current. (1) Switch used to change testing configuration to ground or with a negative DC bias.

The first diagnostic was a charge collecting plate shown in Figure 2.5. Output current was determined using an oscilloscope to measure the voltage developed on a $10 \mathrm{nF}$ capacitor over a 5000 cycle burst. Emission current was then calculated using the current-voltage relation for a capacitor:

$$
\Delta I=C \frac{\Delta V}{\Delta t}
$$

Here $I$ is the emission current created by the thruster, $C$ is the capacitance, $V$ is the voltage measured on the capacitor and $t$ is the time associated with the burst cycle and frequency.

Two configurations of the charge collecting plate were used. In the first configuration, a $1 \mathrm{M} \Omega$ resistor was used to discharge the capacitor during off cycles of thruster operation. Two assumptions were made in this diagnostic setup: 1) the total 
potential difference across the capacitor could be viewed by the Tektronix P5100A (1X) voltage probe before discharging to ground, and 2) all collected current was a result of the plasma discharge with no effect contributed by secondary electrons. Verification of complete discharge by the capacitor between operational cycles was performed by viewing a series of bursts on the oscilloscope and ensuring a return to ground potential between each burst.

The second configuration of the charge collecting plate by replacing the grounded connection with a Glassman EK $10 \mathrm{kV}$ power supply (point 1, Figure 2.5). Applied voltages ranging between -10 to $-1140 \mathrm{~V}$ were used to further extract ion current by creating a negative charge on the plate. This same charge would also work to screen out electrons with energies up to the applied voltage. Current to and from the power supply was limited by using the same $1 \mathrm{M} \Omega$ resistor.

\subsubsection{Faraday Cup with Retarding Potential Analyzer}

The second diagnostic was a retarding potential analyzer with Faraday cup, used to detect charged particle emission similar to that of the current plate. The retarding potential analyzer consisted of three grids used to measure electron and ion energies. The configuration of the Faraday cup is shown in Figure 2.6. 


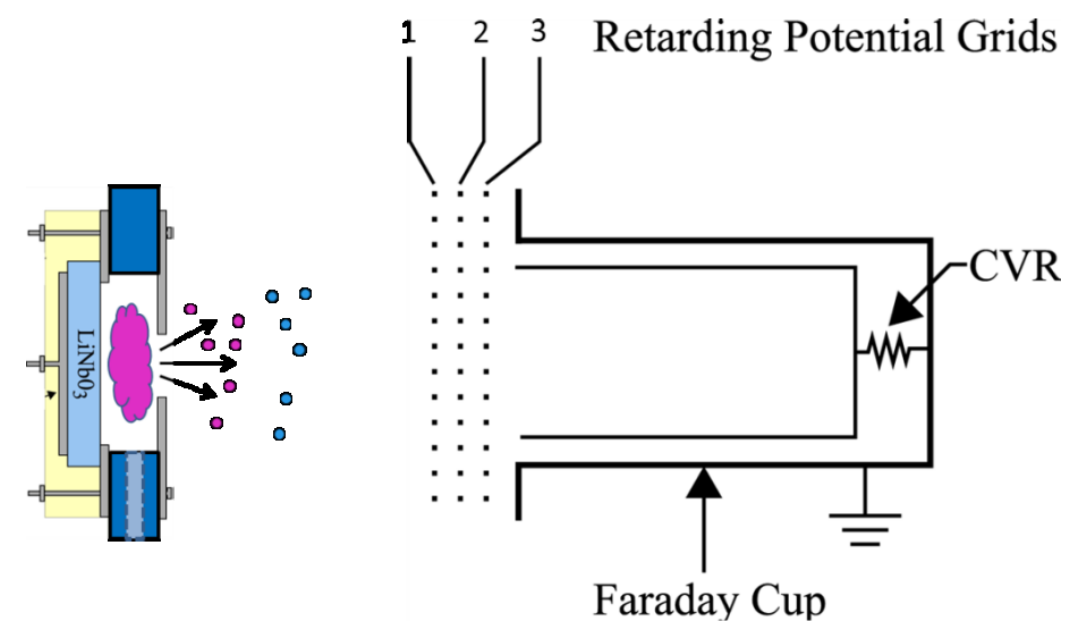

Figure 2.6: Retarding potential analyzer (1) ground grid, (2) bias grid, (3) secondary suppression grid

Grid 1 was used to establish a ground reference. Grid 2 was biased positively or negatively from 0 to $3000 \mathrm{~V}$ to selectively screen electron or ion energies. Grid $3 \mathrm{had}$ a small negative voltage of $-35 \mathrm{~V}$ to suppress any secondary electrons that may emerge from within the Faraday cup. Current emission was then recorded on the oscilloscope utilizing a $1 \mathrm{M} \Omega$ current viewing resistor attached to the rear of the cup. Typically, a correction factor to account for collected charge on the grids $1-3$ is needed to accurately determine the total emission current. However, with an appropriately applied DC voltage, an ion optic effect occurs increasing the relative transparency of the grid as shown in Figure 2.7 [31]. To determine the percentage of ion beam lost to charge collected on the grids, a comparison of the ion current collected on the charge collecting plate described in section 2.3.1 to the ion current collected by the Faraday cup was conducted. The grid transparency could then be calculated according to [31]:

$$
T_{g}=\frac{I_{d}}{I_{b}}
$$


where $T_{g}$ is the effective grid transparency at a selected voltage, $I_{b}$ is the total beam current emitted by the PTPS-MT and $I_{d}$ is the detected beam current at the Faraday cup after passing though the grids. An assumption was made that the total emitted beam current was the same as the current found using the charge collection plate experiments under the same conditions. The measured current in the Faraday cup was $20 \%$ of the charge collecting plate which was then used to account for the true emitted beam current in all Faraday cup measurements.

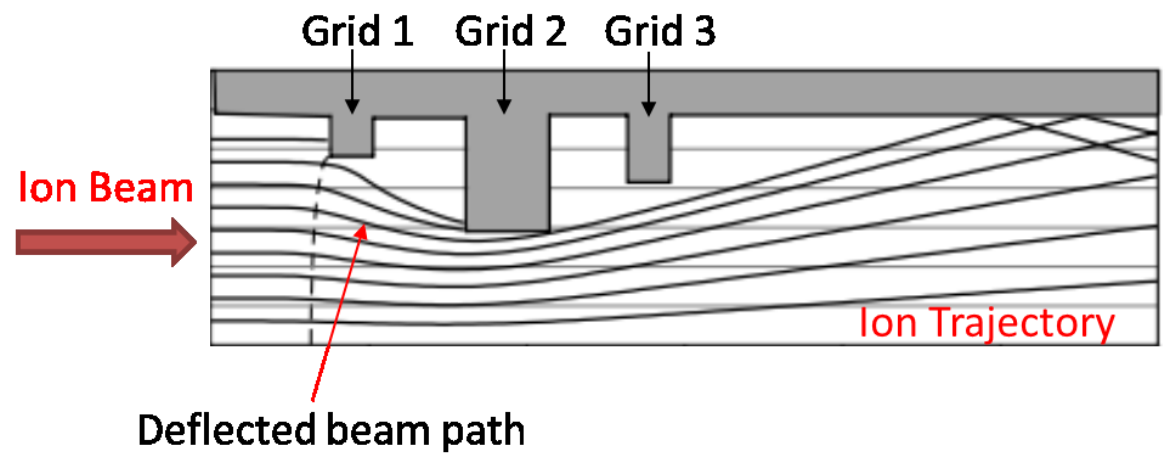

Figure 2.7: Ion trajectory path in biased gridded electric field

To account for beam divergence, a second correction factor was needed. Since the size of the collection grid surface is assumed to be small with respect to beam distribution, not all of the emitted current was detected by the Faraday cup. The percentage of the beam collected by the Faraday cup can be defined by the solid angle, $\Omega$ :

$$
\Omega=\frac{\# \text { particles per second collected in the detector aperture }}{\# \text { particles per second emitted by the source }}
$$

For a disk source parallel to a circular aperture, the solid angle can be found according to [33]: 


$$
\Omega=\frac{\omega^{2}}{4}\left\{\begin{array}{c}
1-\frac{3}{4}\left(\delta^{2}+\omega^{2}\right)+\frac{15}{8}\left(\frac{\delta^{2}+\omega^{2}}{3}+\delta^{2} \omega^{2}\right) \\
-\frac{35}{16}\left[\frac{\delta^{6}+\omega^{6}}{4}+\frac{3}{2} \delta^{2} \omega^{2}\left(\delta^{2}+\omega^{2}\right)\right]
\end{array}\right\}, \delta=\frac{R_{s}}{d} \text { and } \omega=\frac{R_{d}}{d}
$$

Where $R_{s}$ is the radius of the source aperture, $R_{d}$ is the radius of the detector, and $d$ is the distance between the detector and the source as shown in Figure 2.8:

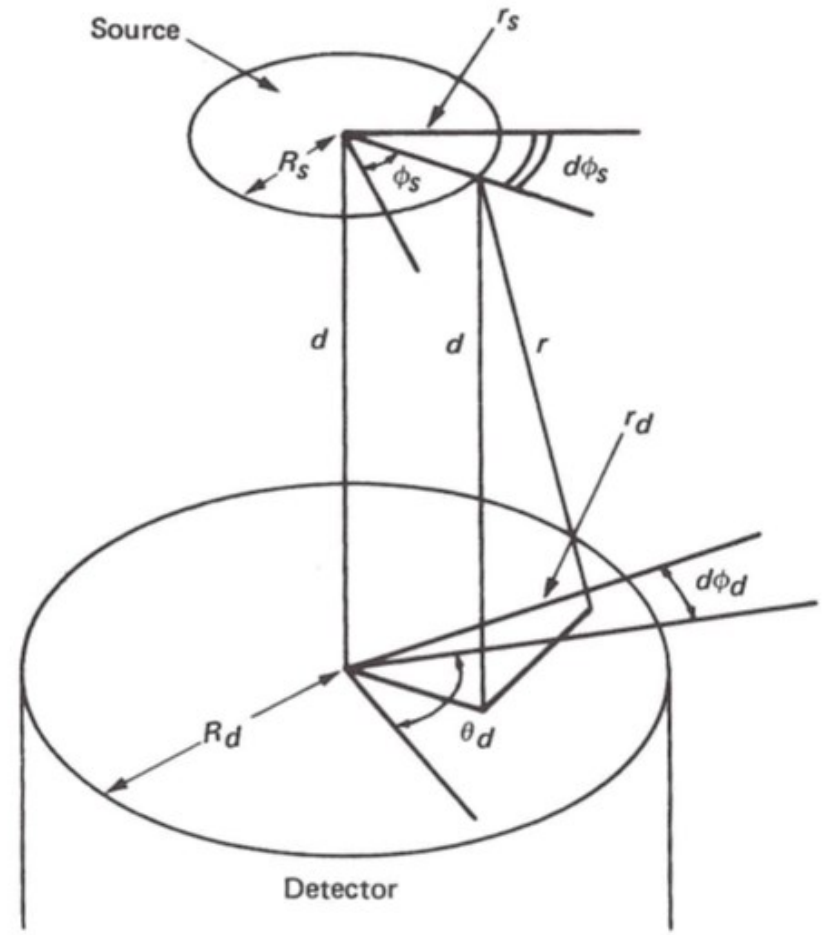

Figure 2.8: Solid angle for a disc source and a detector with a circular aperture [33].

If the disc source is significantly small compared to the detector window, a simplified formula for a point source can be used:

$$
\Omega=\frac{1}{2}\left(1-\frac{d}{\sqrt{d^{2}-R_{d}^{2}}}\right)
$$

Comparison of Equation 2.13 and 2.14 for the detector parameters used during testing agree to less than $1 \%$ difference. Therefore, Equation 2.10 was used for all calculations. 
Ion transit times, used in the calculation of thrust, were measured using the Faraday cup placed at distances between $1 \mathrm{~cm}$ and $5 \mathrm{~cm}$. Previous experiments show plasma forming twice during each RF cycle, occurring at the maximum and minimum points on the voltage waveform [43]. Measurement of ion transit time was made by taking the peak of the RF voltage signal to the start of ion collection within the Faraday, as shown in Figure 2.9.

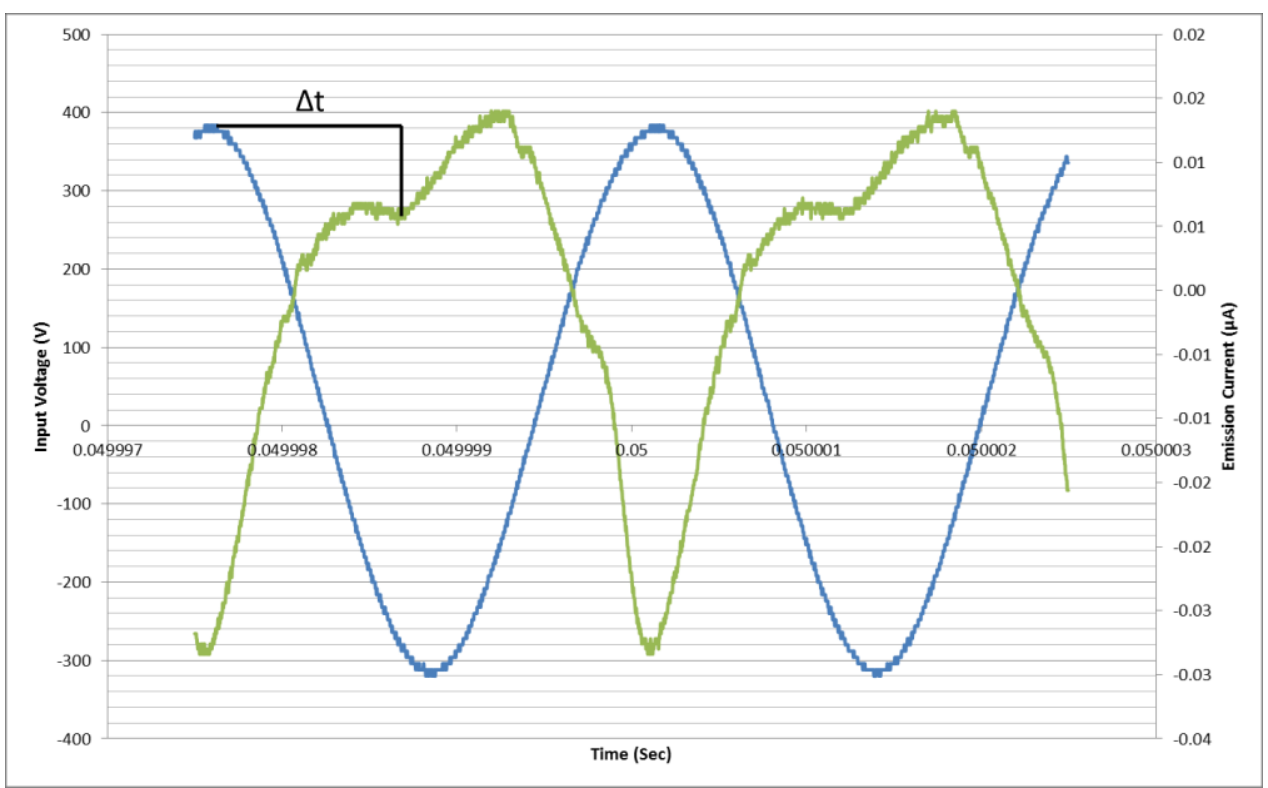

Figure 2.9: Calculation of ion transit time using Faraday cup output.

Velocities were then calculated using:

$$
V=\frac{\Delta d}{\Delta t}[\mathrm{~m} / \mathrm{s}]
$$

where $d$ is the distance from the thruster aperture to the start of the Faraday cup and $t$ is the ion transit time. 


\subsubsection{Thermocouple}

The third diagnostic was an Omega Type K nickel-chromium, nickel-aluminum

thermocouple capable of measuring temperatures from -200 to $1250^{\circ} \mathrm{C}$. The sensing probe of the thermocouple was attached to the output electrode on the surface of the lithium niobate disc. A nickel-chromium, nickel-aluminum vacuum feed through was used to remove the need for thermal circuit calculations with an ice bath reference when dissimilar metals are used. Temperature measurements were then conducted by monitoring a 38XR-A Amprobe during operation of the PTPS-MT. 


\section{Chapter 3}

\section{Simulations}

This chapter discusses COMSOL simulations used to refine previous designs of the PTPS-MT. The first section will discuss the simulation design and parameters used to model the PTPS-MT. The second half of the chapter will highlight optimized results that were then used in experimental testing. All simulations were restricted to a $10 \mathrm{~mm}$ and $20 \mathrm{~mm}$ diameter crystal due to lab availability. The results from the simulations could then be verified through bench top experimentation.

\subsection{Simulation Design}

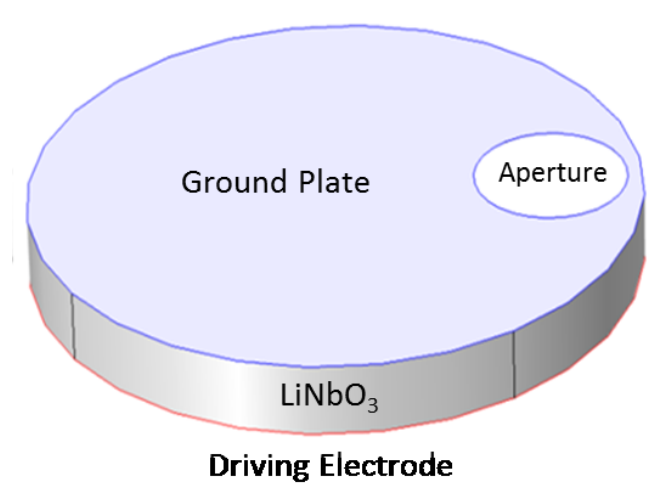

(a)

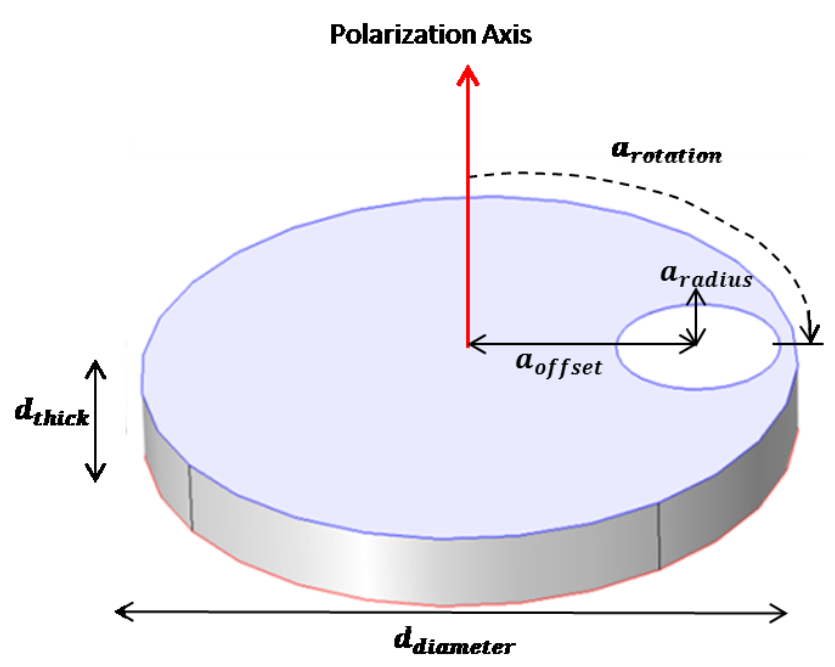

(b)

Figure 3.1: (a) 3D model with ground electrode applied (blue) and driving electrode (red). (b) Definition of parameters used in simulations 
A dimensionally correct model of the lithium niobate used in the PTPS-MT was developed in COMSOL and is shown in Figure 3.1. Two sizes of the disc were modeled: a $10 \mathrm{~mm}$ diameter and a $20 \mathrm{~mm}$ diameter. Thicknesses were varied from $1 \mathrm{~mm}$ up to the diameter of the disc. Properties of the crystal were then modified according to previous research to better match the simulation with experimental results [25] Table 3.1 summarizes the model parameters used in the simulations.

Table 3.1 Summary of model parameters for $45^{\circ}$ Z-Cut Crystal.

\begin{tabular}{|l|l|}
\hline Parameter & Value \\
\hline Dialectric Loss Tangent & $\delta=0.0013$ \\
\hline Mechanical Loss Factor (Meas.) & $\eta=1 / 450$ \\
\hline Permittivity & $\varepsilon^{S}{ }_{11}=38.9, \varepsilon^{S}{ }_{33}=32.3$ \\
\hline Elastic Stiffness $\left(\times 10^{10} \mathrm{~N} / \mathrm{m}^{2}\right)$ & $\begin{array}{l}c^{E}{ }_{11}=20.3, c^{E}{ }_{12}=7.3, \\
c^{E}{ }_{14}=1.1, c^{E}{ }_{33}=21.8, c^{E}{ }_{44}=7.4, c^{E}{ }_{66}^{E}=7.7\end{array}$ \\
\hline Peizoelectric Coeff. $\left(\times 10^{-12} \mathrm{C} / \mathrm{N}\right)$ & $d_{22}=33.8, d_{31}=14, d_{33}=18.4, d_{15}=77$ \\
\hline Coupling Factor $(\%)$ & $k_{23}=48.4, k_{33}=51.2$ \\
\hline Local Coordinate Rotation & $45^{\circ}$ about Global X axis \\
\hline Disk Diameter & $10 \mathrm{~mm}, 20 \mathrm{~mm}$ \\
\hline Disk Thickness & $1 \mathrm{~mm}-20 \mathrm{~mm}$ (varied) \\
\hline Aperture Offset & $0 \mathrm{~mm}-8 \mathrm{~mm}$ (varied) \\
\hline Aperture Radius & $0.5 \mathrm{~mm}-3.5 \mathrm{~mm}$ (varied) \\
\hline Aperture Rotation & $0-360^{\circ}$ (varied) \\
\hline
\end{tabular}

Simulations in COMSOL used a rotated local axis, assigned to the lithium niobate crystal, to account for the effects of the rotated polarization axis relative to the global axis. The electrodes and applied fields used the global axis. For verification of this procedure and COMSOL results, externally calculated parameters utilizing MATLAB 
were conducted for one rotation. Values obtained from MATLAB agreed with the COMSOL results for a $45^{\circ}$-rotated polarization axis and are listed in Table 3.1.

\subsubsection{Dielectric Loss Tangent}

The dielectric loss tangent, $\delta$, is a parameter that was used to represent electrical loss in a material for ac signals. The reported loss tangent for lithium niobate is 0.0013 , for temperatures ranging from $293 \mathrm{~K}$ to $573 \mathrm{~K}$, and frequencies ranging up to $9 \mathrm{GHz}$, which are within the operating range of the PTPS-MT [20]. In COMSOL the loss tangent is accounted for by modifying the materials dielectric constant, $\epsilon^{\mathrm{S}}$, according to the equations:

$$
\begin{gathered}
\eta_{\epsilon^{S}}=\epsilon_{r} * \tan \delta \\
\epsilon_{S}^{(i, j)}=\left(1-j \eta_{\epsilon^{S}}(i, j)\right) \in_{S}{ }^{(i, j)}
\end{gathered}
$$

Application of the loss tangent reduced the reported permittivity of 29.2 down to 28 , which was the value used in simulations.

\subsubsection{Mechanical (Structural) Loss Factor}

Physical properties of the crystal, electrodes, and ceramic holder result in losses that reduce the effective energy delivered by the power supply. Materials, such as the crystal and metal electrodes, have well documented mechanical quality factors, $Q_{m}$, that are accounted for in COMSOL through the modification of the elastic constant, $c_{E}$. Other factors, such as the physical tensioning of electrodes to hold the crystal in place, are difficult to determine analytically, requiring experimentation to account for these parameters on a case by case basis. 
Generally, once determined, these losses are assumed to remain constant over many experiments, only changing when the crystal was removed from the ceramic holder. To maintain a consistent mechanical loss between experiments with different crystals, an iterative process was conducted of adjusting the tension on the screws until the same frequency corresponding to the minimum impedance was achieved. By matching the resonant frequency, it was assumed that the mechanical loss was the same, according to:

$$
Q_{m} \approx \frac{\omega_{r}}{2\left(\omega_{a}-\omega_{r}\right)} \frac{\left(1+\frac{\left|Z_{\max }\right|}{\left|Z_{\min }\right|}\right)}{\sqrt{\mid \frac{\left|Z_{\max }\right|}{\left|Z_{\min }\right|}}}
$$

This equation was proposed by Ikeda as a method to estimate the mechanical quality of a resonator through analysis of the impedance measurements at resonance and antiresonance points [20]. If, after changing a crystal, the impedance plot did not match, mechanical loosening or tensioning of the screws could shift the plot to the right or left with respect to frequency by changing the mechanical quality as shown in Figure 3.3.

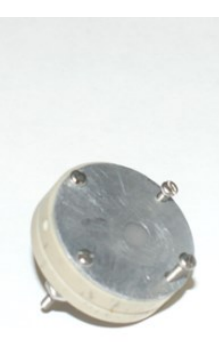

(a)

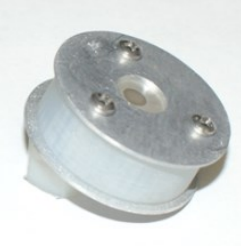

(b)

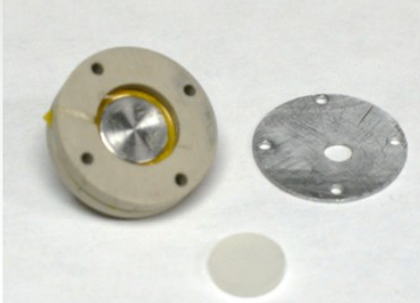

(c)

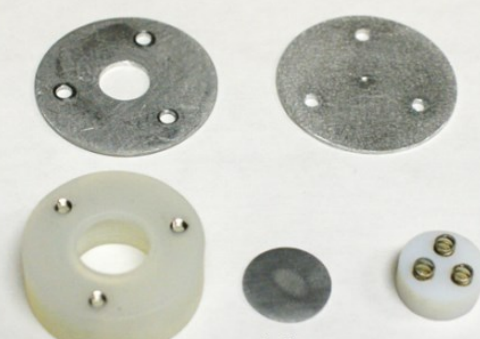

(d)

Figure 3.2: Comparison of piezoelectric holders used in experimentation. (a) Early holder using screw tensioning, (b) Final holder using spring pressure, (c) Early holder disassembled, (d) Spring holder disassembled. 
Two mechanical holders were used in experimentation (Figure 3.2). The first holder, used during the majority of the experiments during early testing, used the mechanical tensioning of screws to hold a crystal in place, as described earlier. This holder had consistently low Q values, ranging from 50-75. During the later stages of experimentation, a new method of mounting the crystal with springs resulted in a mechanical quality factor of 400 or more. The spring method allowed for consistent and repeatable results. The measured $\mathrm{Q}_{m}$ factor from the spring-loaded holder was used in COMSOL modeling. This was then applied to the COMSOL model through a structural loss factor, $\eta_{s}$, which would modify the elastic constant of the system via Equation 3.2 and 3.3. The mechanical loss is assumed to act isotropically, thereby removing the tensor terms.

$$
\begin{aligned}
& \eta_{s}=\frac{1}{Q_{m}} \\
& c_{E}=\left(1+j \eta_{s}\right) c_{E}
\end{aligned}
$$
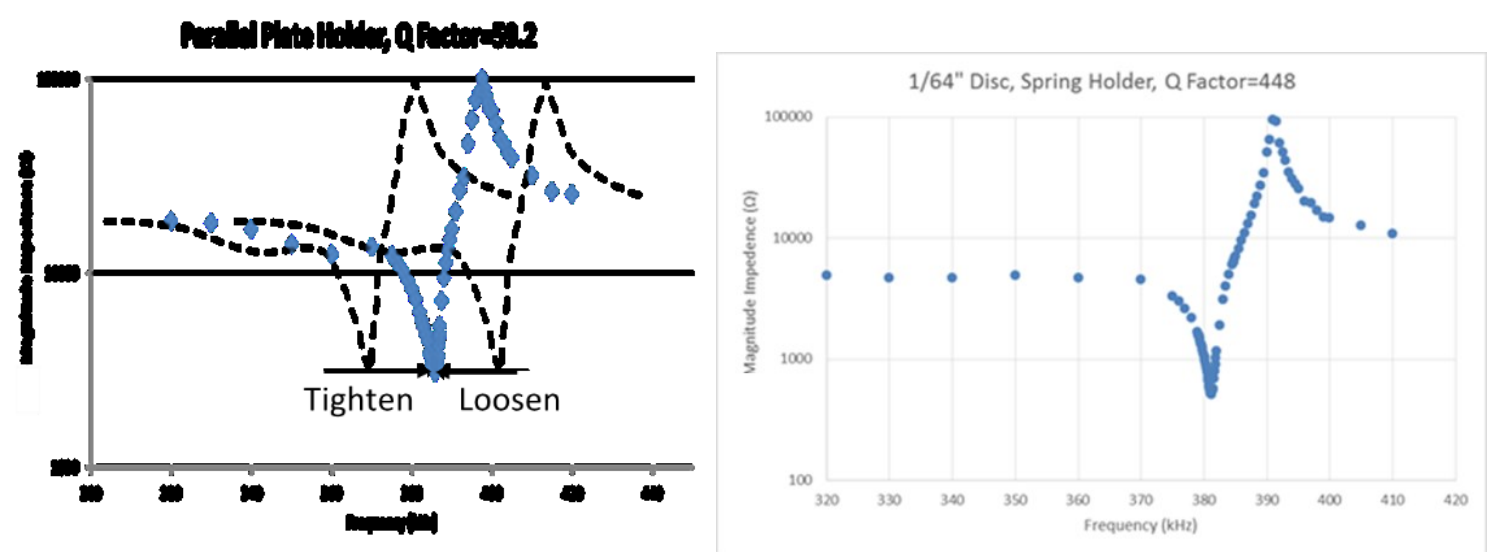

Figure 3.3: (a) Plot showing adjustment of mechanical quality through tensioning of electrodes holding piezoelectric disc. (b) Impedance plot of spring tensioned holder requiring no adjustment. 


\subsubsection{Local Coordinate System}

Simulation of a radially symmetric uniform crystal with a linear polarization normal to the disc surface can be accomplished in a simple 2D model, as the geometry around one axis would always be the same. However, the crystals used in the PTPS-MT have an anisotropic polarization in the direction of the $\mathrm{X} 3$ axis, requiring all modeling to be performed in a three-dimensional space. The global axis, represented by $\mathrm{X}, \mathrm{Y}$, and Z in Figure 3.4, establish a coordinate system in which the electric fields and mechanical forces are defined. The rotated local coordinate system, represented by X1, X2, and X3 modify the lithium niobate crystal tensors relative to the global axis to properly account for changes in the boundary conditions for simulation.

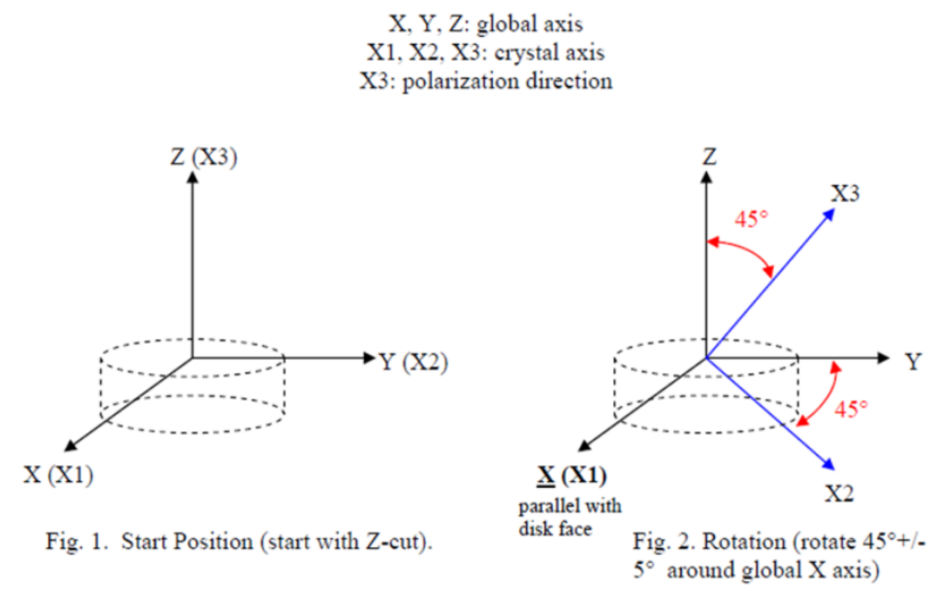

Figure 3.4 (1) Standard crystal model with polarization direction in global $\mathrm{Z}$ axis direction (2) Rotated polarization of crystal used in experiments.

A cross-sectional plot of the crystal in COMSOL showing the displacement under an applied voltage demonstrates application of the local coordinate system used in modifying the piezoelectric polarization axis. 


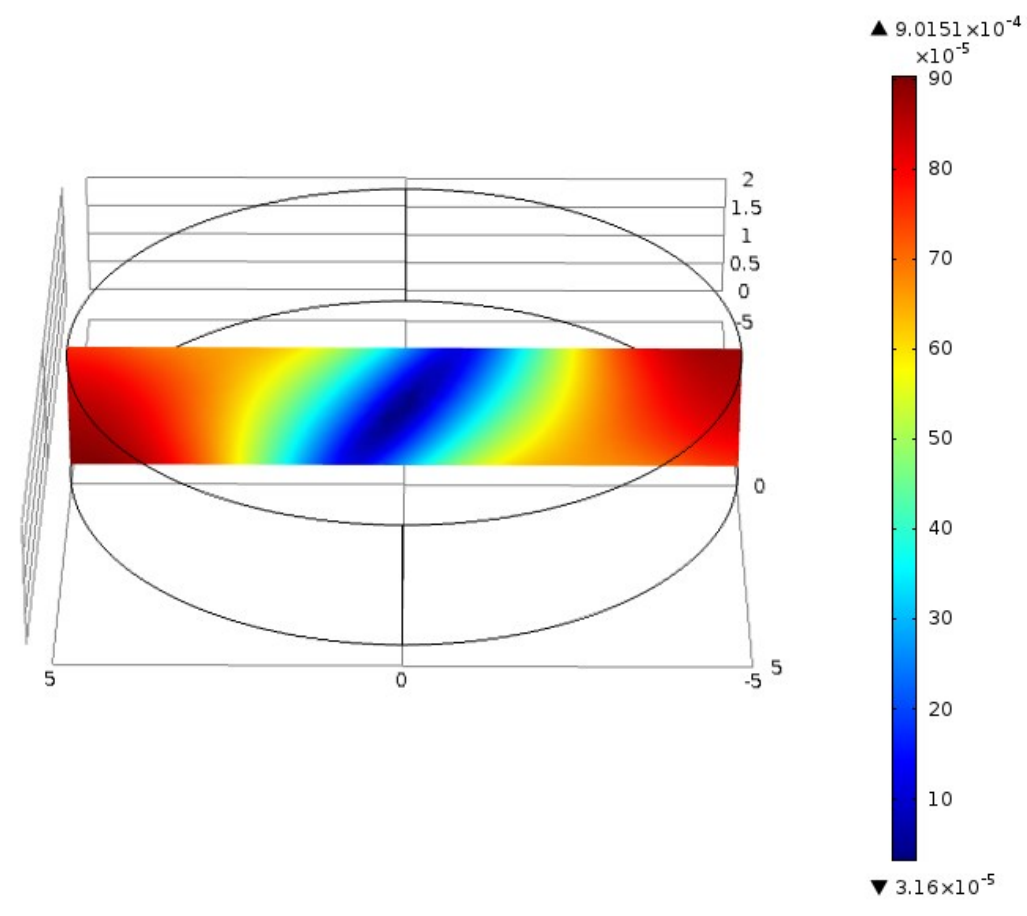

Figure 3.5 Cross sectional plot of piezoelectric disc showing displacement corresponding to the rotated local axis (global axis shown for reference). Blue slant is due to polarization angle.

\subsubsection{Equivalent Tensile Stress}

Von Mises stress is used to predict the strain of materials under multi-axial loading. This parameter was chosen to remove directionality and comparison of individual stress tensors by using a single scalar stress value to determine the yield point. The von Mises stress is calculated through principal stresses, $\sigma 1, \sigma 2, \sigma 3$, corresponding to a defined coordinate system, through Equation 3.4:

$$
\sigma_{v}=\sqrt{\frac{1}{2}\left[\left(\sigma_{1}-\sigma_{2}\right)^{2}+\left(\sigma_{1}-\sigma_{3}\right)^{2}+\left(\sigma_{2}-\sigma_{3}\right)^{2}\right]}
$$


One goal of the crystal simulation was to determine if changes to crystal dimensions could be used to reduce the von Mises stress while still achieving a high transformation ratio capable of producing plasma. For lithium niobate, the yield stress is documented to be between 30-120 MPa depending on the crystal structure corresponding to the direction of applied stress. [34]

\subsection{Simulation Results}

Simulation of the PTPS-MT was conducted to confirm previous experimental findings and determine if modifications to the aperture placement and crystal dimensions could increase plasma formation and reduce failure due to fracture. Previous research had shown an aperture offset of $2 \mathrm{~mm}$ to provide the best results for reliable plasma formation [25]. It was assumed that the aperture offset was a result of the rotated $45^{\circ}$ polarization axis and was needed to match the resulting displacement and electric profile of the disk. (Figure 3.5). This reasoning did not hold true when crystals with larger thicknesses and diameters were used, requiring further investigation. Additionally, bench top experiments showed a wide range of ground aperture locations capable of developing a plasma discharge of varying size and intensity. Visually, it was difficult to determine which location provided the best plasma discharge, requiring a numerical simulation to determine the optimal placement of the ground aperture.

Three simulations were conducted to understand the effects of aperture placement on the piezoelectric disk and determine where the highest voltage transformation could be obtained: ground aperture offset, ground aperture diameter and rotation around disc 
surface. A high transformation ratio could be used to reduce the input voltage required to produce a high potential in the output aperture, which would effectively result in a smaller power plant for space applications.

A fourth simulation was conducted to investigate if changing crystal dimensions could be used to reduce the von Mises stress while still producing a high-voltage output capable of ionizing a background gas. Crystals used in the lab experienced a number of failures due to fracture when operating at extreme conditions of voltage input or high duty cycles. However, these conditions also produced the greatest results for thrust, making it important for investigating ways that could reduce these failures. Reducing the stress would be important for long term operation and reliability if this thruster design were to be viable for space missions.

To simulate operation of the PTPS-MT, a $100 \mathrm{~V}_{\mathrm{pp}}$ electrical RF pulse was applied to the base of the crystal and corresponding output voltages within the grounded aperture plate were measured to determine the voltage transformation ratio. Aperture size, radial offset, angular rotation, crystal diameter, and crystal thickness were swept and resultant resonance frequencies were determined to achieve the largest voltage output.

\subsubsection{Effect of Aperture Diameter and Offset on Transformation Ratio}

Based on visual observations of plasma formation from bench top experiments, attempts at distinguishing an optimal ground aperture placement were educated guesses. Previous works reported that a radial offset of the ground aperture of $2 \mathrm{~mm}$ provided the best results, but it was unknown how this offset was placed relative to the polarization 
axis, due to crystals arriving unmarked from the manufacturer [25]. It was also noted that while an offset of $2 \mathrm{~mm}$ provided good results, this placement did not need to be precise. It was observed that a large range of locations also provided similar results. A numerical simulation was conducted to precisely determine the effects of varying the location and diameter of the ground plate aperture across the surface of the lithium niobate disk. A range of diameters from $0.5 \mathrm{~mm}$ to $3.0 \mathrm{~mm}$ were selected. The apertures were simulated starting at the center of the piezoelectric disc and moving radially outward $0.5 \mathrm{~mm}$ each iteration. An experimentally determined resonant frequency of $385 \mathrm{kHz}$ was initially used for all parameters with tuned resonant frequencies being introduced during subsequent simulations. Results for the aperture offset of the $10 \mathrm{~mm}$ diameter, $2 \mathrm{~mm}$ thick (hereby referred to as $10 \times 2 \mathrm{~mm}$ ) crystal are shown in Figure 3.6.

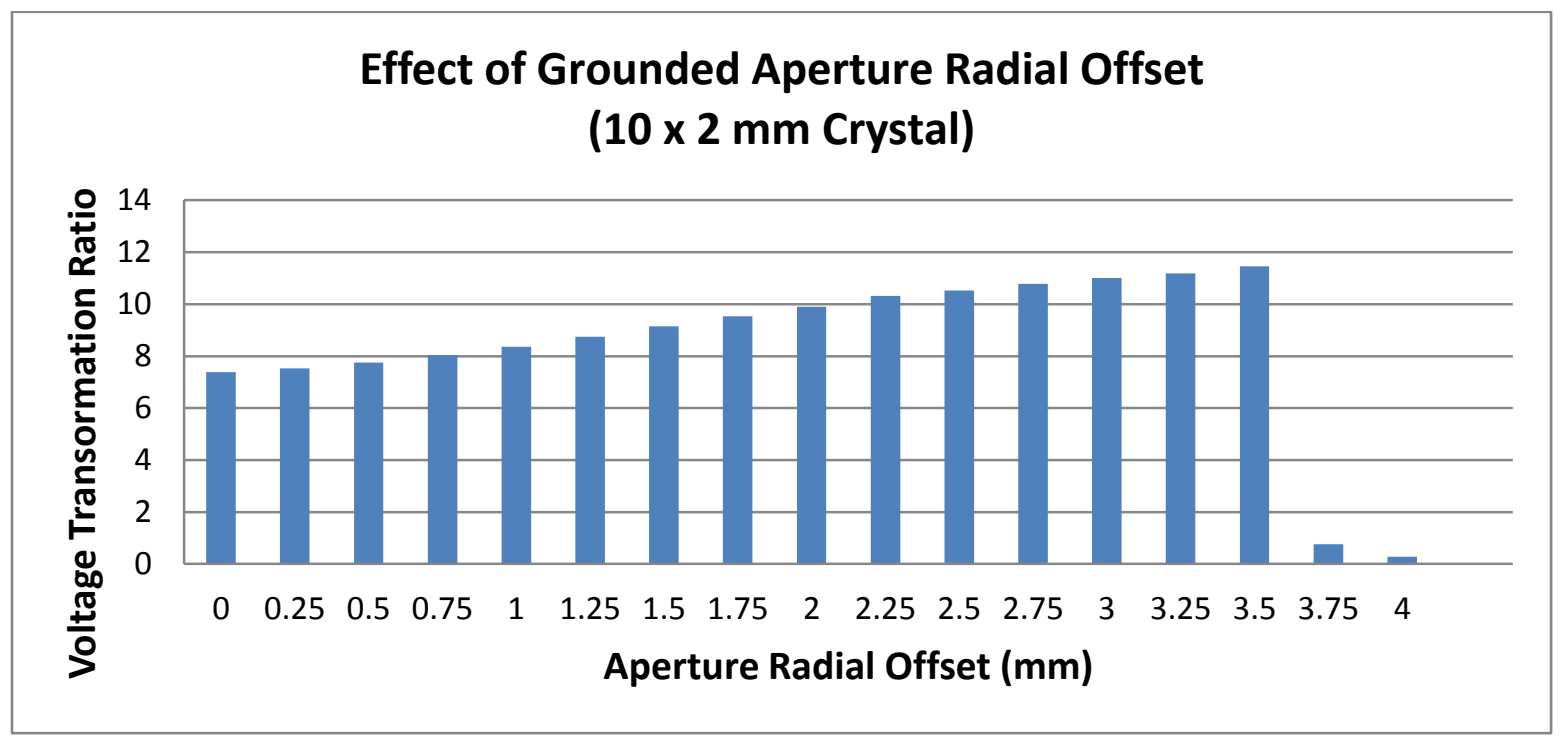

Figure 3.6: Effect of aperture offset on transformation ratio for a $10 \mathrm{~mm}$ diameter crystal. Ground plate aperture diameter of $2.5 \mathrm{~mm}$ used for all locations. 
As the radial offset moved outward along the disc radius, transformation ratios increased until the offset reached approximately $70 \%$ of the disc radius. The numerical simulations show an increase of $15 \%$ in the transformation ratio by increasing the radial offset of the ground plate aperture to $3.5 \mathrm{~mm}$ over the previously reported optimal offset of $2 \mathrm{~mm}$. The same simulation was conducted with the $20 \mathrm{~mm}$ diameter, $4 \mathrm{~mm}$ thick (20 x $4 \mathrm{~mm}$ ) crystal. Results are shown in Figure 3.7.

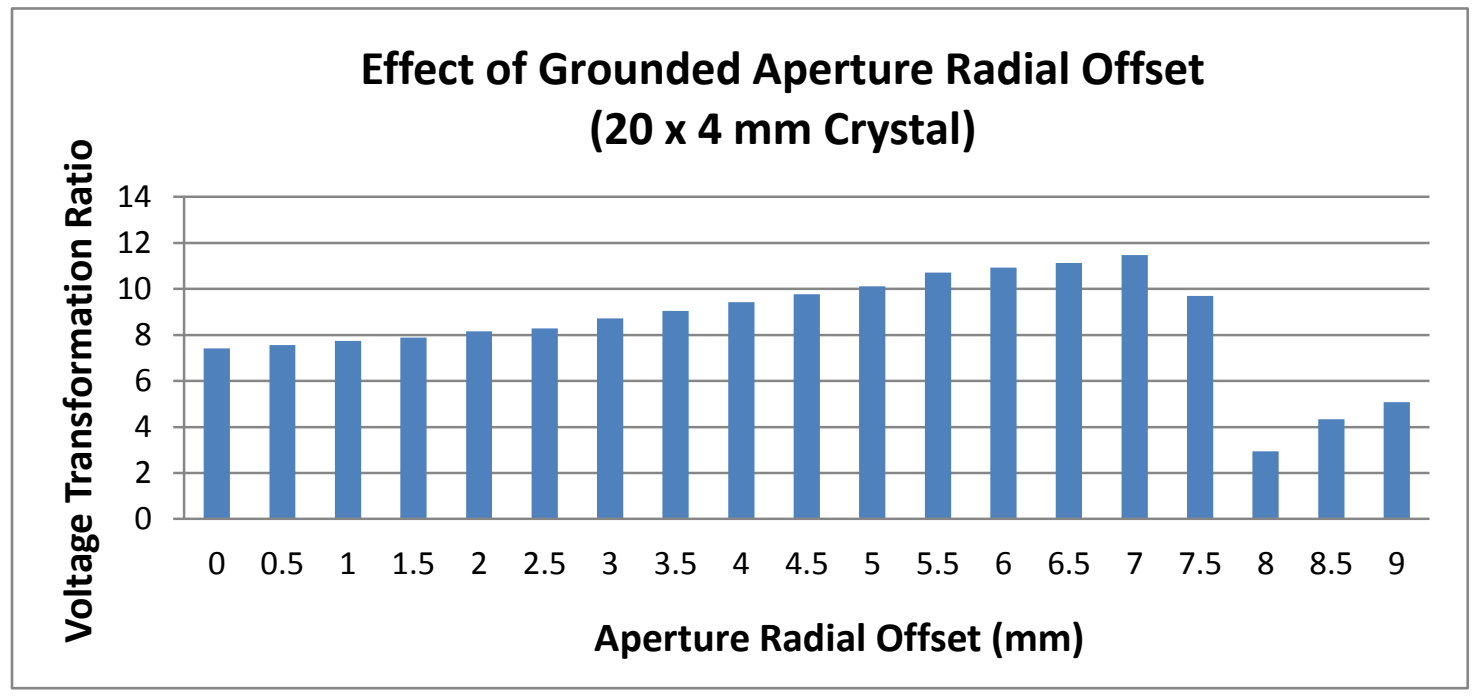

Figure 3.7: Effect of aperture offset on transformation ratio for a $20 \mathrm{~mm}$ diameter crystal. Ground plate aperture diameter of $5 \mathrm{~mm}$ used for all locations.

Again, the highest voltage transformation occurs at offset corresponding to $70 \%$ of the disc radius. This can partially be explained by the large displacement of the crystal exhibited during the radial resonance mode. When the crystal was operated in radial resonance, the outer edges exhibit a large displacement relative to the center of the crystal, as shown in Figure 3.8. This large displacement results in a high strain being 
induced in the crystal structure which is then coupled into a higher electric field according to the direct piezoelectric effect found in Equation 2.1. The large drop in voltage transformation occurred when the fixed diameter grounded aperture began to exceed the radius of the lithium niobate crystal during radial offset iterations. Follow on simulations were conducted to adjust the ground plate aperture diameter for these larger offsets.

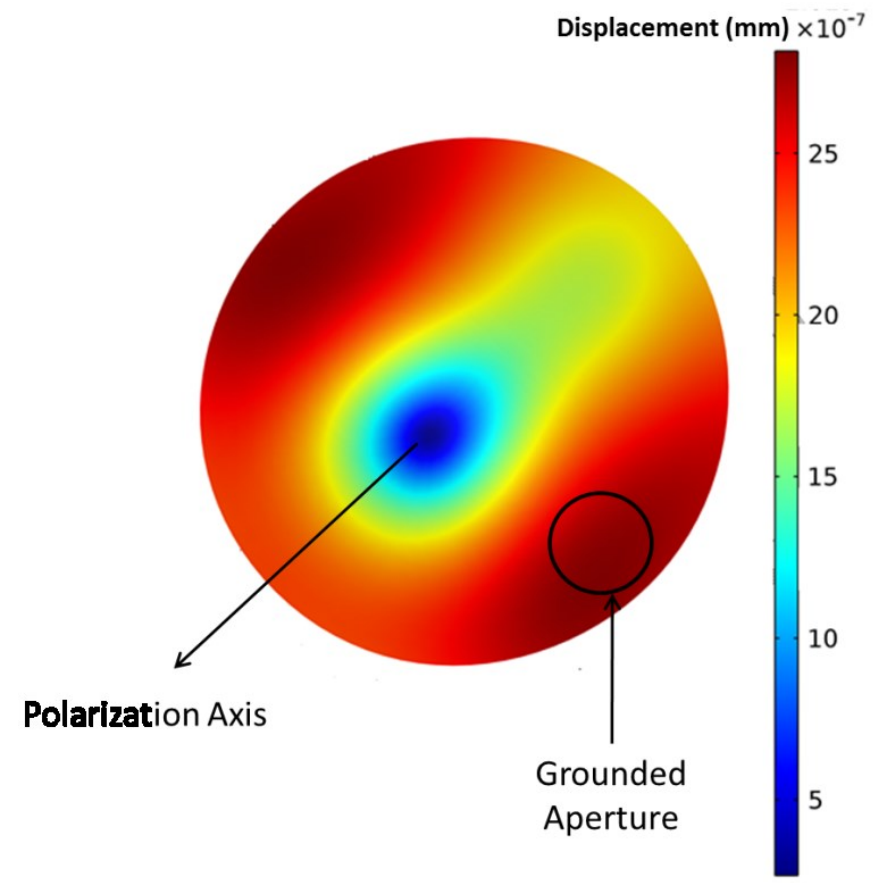

Figure 3.8: Displacement of piezoelectric disc corresponding to ground aperture location of greatest output. Darker red corresponds to greater displacement. Displacement not radially symmetric due to polarization axis rotation.

The next series of simulations were conducted to determine if the diameter of the grounded plate aperture had an impact on the transformation ratio. A parametric sweep of offset and diameter was conducted where the aperture diameter was varied over the range of 0.5 to $1.75 \mathrm{~mm}$ and the offset was repeated over the range of $60-80 \%$ of the disc 
radius. Results from adjusting the grounded plate aperture diameter are shown in Figure 3.9.

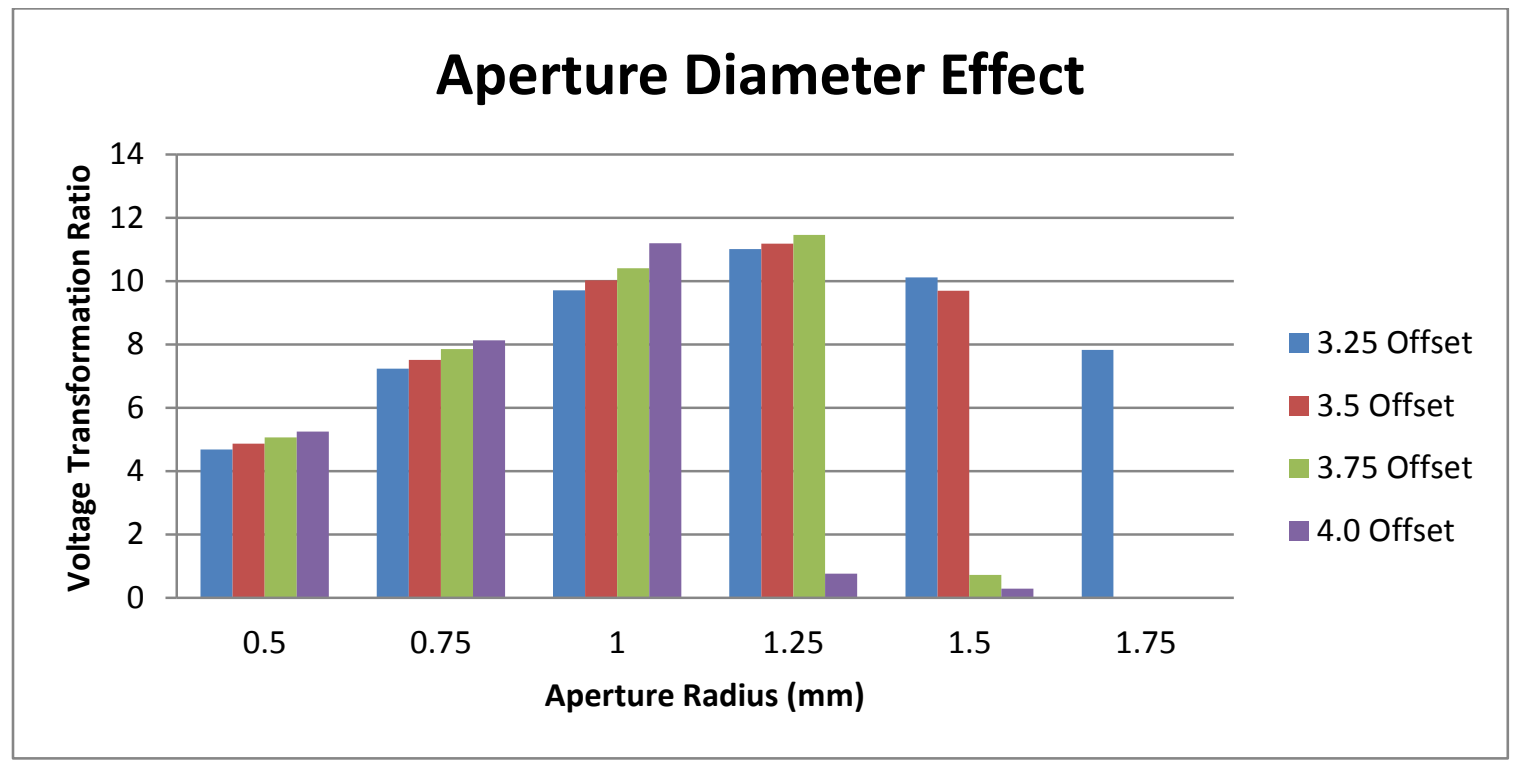

Figure 3.9: Effect of aperture diameter on voltage transformation for $10 \times 2 \mathrm{~mm}$ disc.

An aperture diameter of $1.25 \mathrm{~mm}$, corresponding to $25 \%$ of the crystal's radius, consistently had the highest voltage transformation with sensitivity to the size and location being very important. Both crystals exhibited the same trend, with the $20 \mathrm{x} 4 \mathrm{~mm}$ disc achieving the highest transformation with a $2.5 \mathrm{~mm}$ aperture.

\subsubsection{Frequency Effect on Transformation Ratio}

At resonance, the impedance of the piezoelectric is significantly reduced as the cycling frequency of the RF input closely matches the mechanical resonance of the disc. By plotting the impedance of the disc, the resonance and anti-resonance points can be seen in Figure 3.8. 


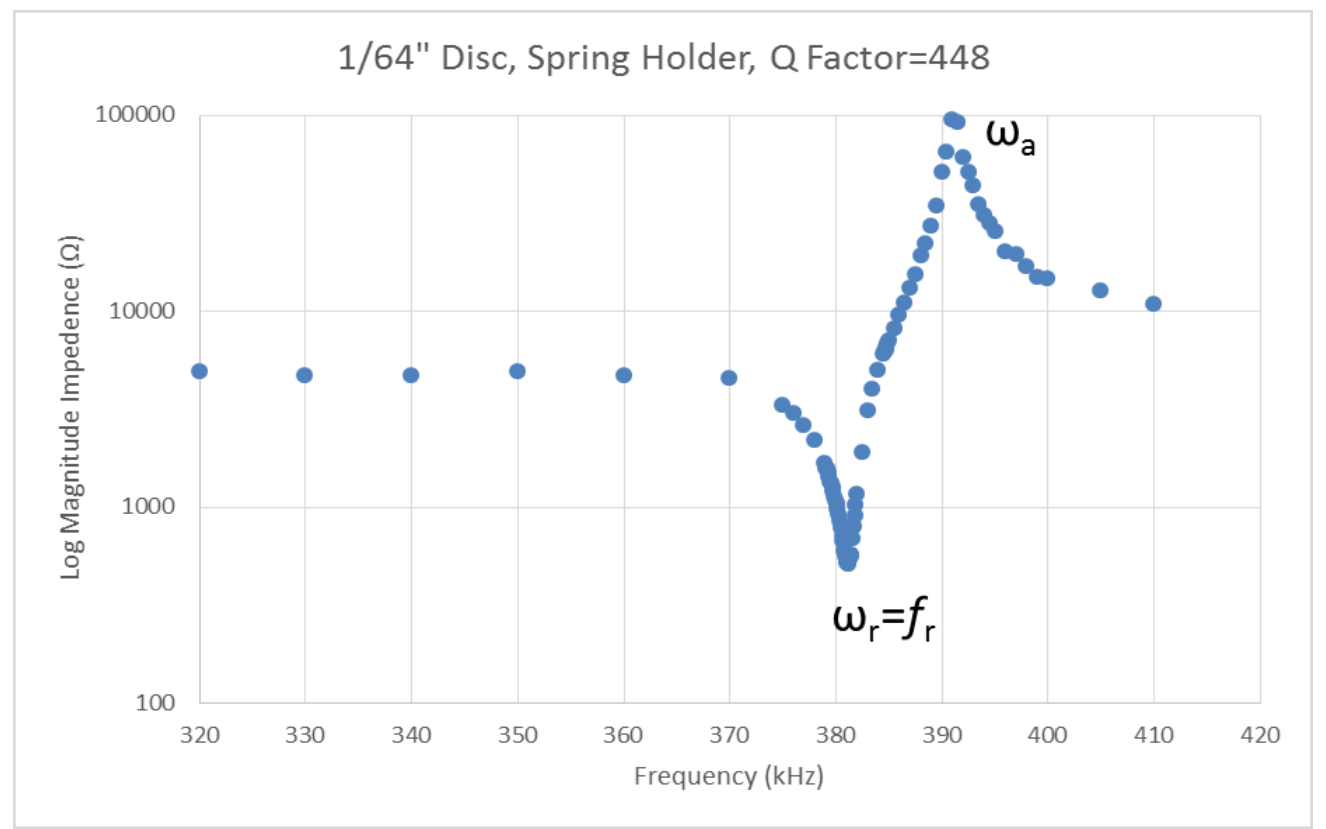

Figure 3.10: Measured impedance plot showing resonance and anti-resonance frequencies of a piezoelectric disc.

If mechanical losses are ignored, the frequency for the minimum impedance, $f_{m}$, is equal to the resonance frequency of crystal. The sensitivity of the appropriate RF selection is shown in Table 3.2, where it can be seen that a shift from the resonant frequency by as little as $0.005 \%$ can reduce the voltage transformation by as much as $60 \%$. This becomes important during operation of the thruster, where it is important to precisely locate the crystal resonance for optimal operation. 
Table 3.2: Voltage transformation sensitivity to shift in frequency.

\begin{tabular}{|l|l|}
\hline \multicolumn{1}{|c|}{ Frequency } & \multicolumn{1}{c|}{$\begin{array}{c}\text { Voltage } \\
\text { Transformation }\end{array}$} \\
\hline 395.6 & 4.92 \\
\hline 395.7 & 5.44 \\
\hline 395.8 & 6.08 \\
\hline 395.9 & 6.88 \\
\hline 396 & 7.91 \\
\hline 396.1 & 9.17 \\
\hline 396.2 & 10.41 \\
\hline 396.271 & 11.48 \\
\hline 396.3 & 10.21 \\
\hline 396.4 & 4.41 \\
\hline 396.5 & 0 \\
\hline 396.6 & 0 \\
\hline
\end{tabular}

\subsubsection{Effect of Ground Electrode Rotation on Transformation Ratio}

The third set of simulations used the optimized grounded aperture design to consider the effect of rotating the aperture across the surface of the disc. Based on the COMSOL model, the rotation was applied to the crystal by changing the rotation for $\alpha$ on the local axis applied to the lithium niobate crystal, as shown in Figure 3.11. This was equivalent to rotating the ground aperture around the surface of the disc.

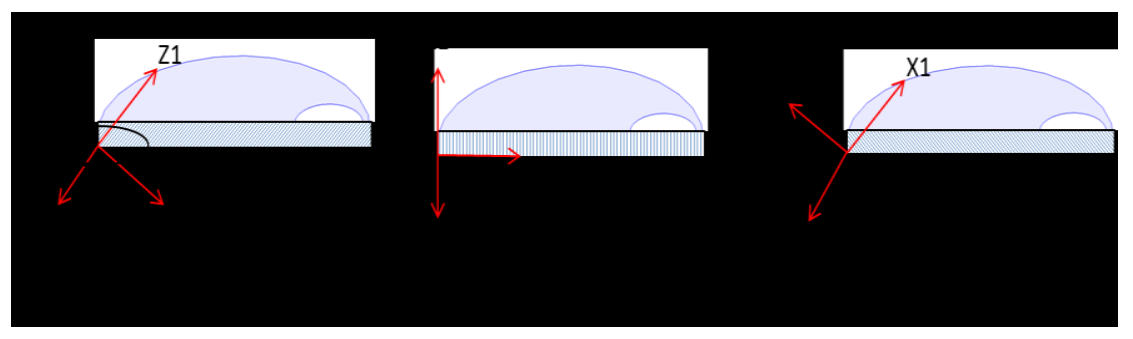

Figure 3.11: Rotated local coordinate system showing cross section of crystal polarization relative to ground aperture. 
For both the $20 \times 4 \mathrm{~mm}$ and $10 \times 2 \mathrm{~mm}$ disc, the optimal voltage transformation occurred at $100^{\circ}$ and $260^{\circ}$ off of the crystal polarization axis as shown in Figure 3.12 and 3.13. These points corresponded to the greatest displacement on the disc. While plots for both the 10 and $20 \mathrm{~mm}$ crystals follow the same trend, a 50\% reduction of von Mises stress for the same voltage transformation occurred in the larger diameter. This was important since the peak transformations in the $10 \mathrm{~mm}$ diameter crystal correspond to stresses that potentially exceed the yield strength of lithium niobate. Achieving a high transformation with low stress would be important for long term operation where a high reliability is important.

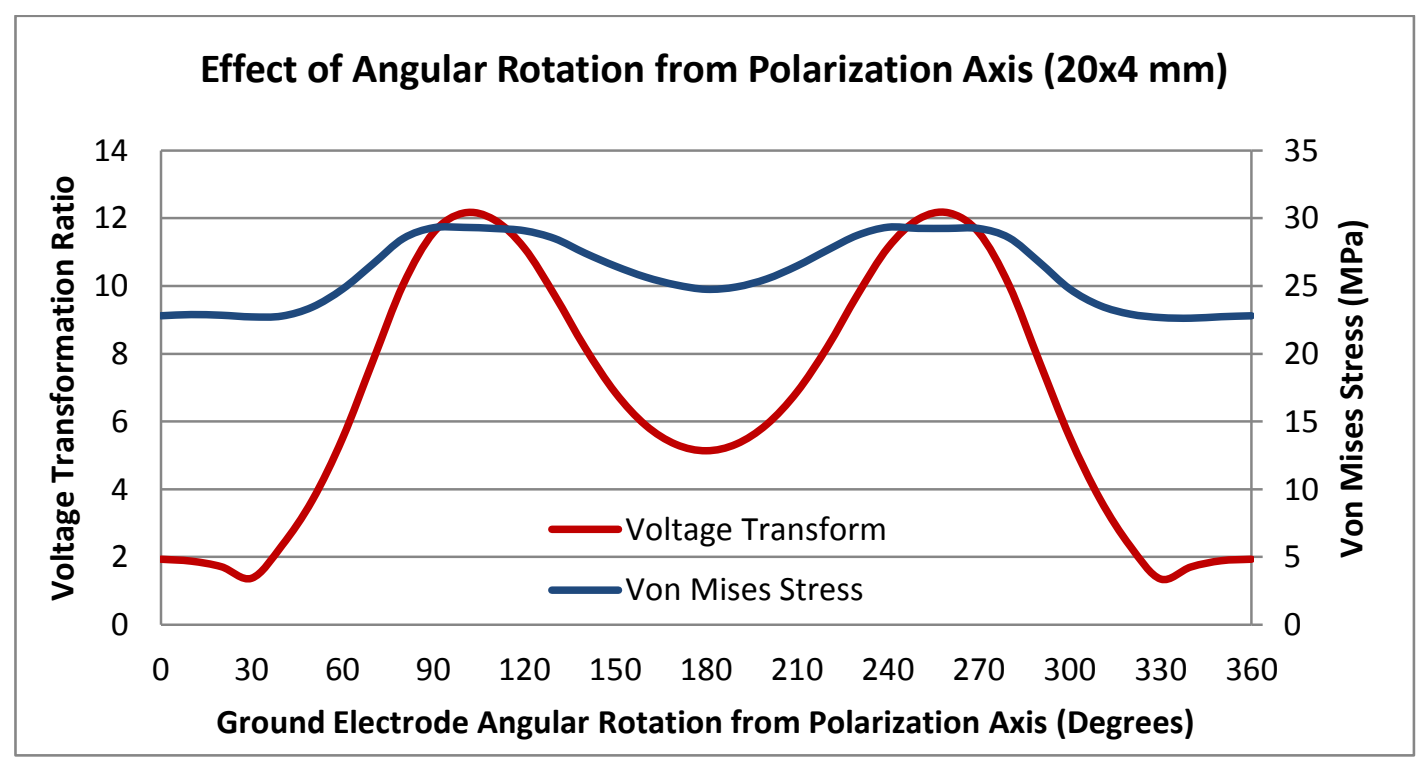

Figure 3.12: Effect of ground plate aperture rotation across surface of disc $(20 \times 4 \mathrm{~mm}$ crystal) 


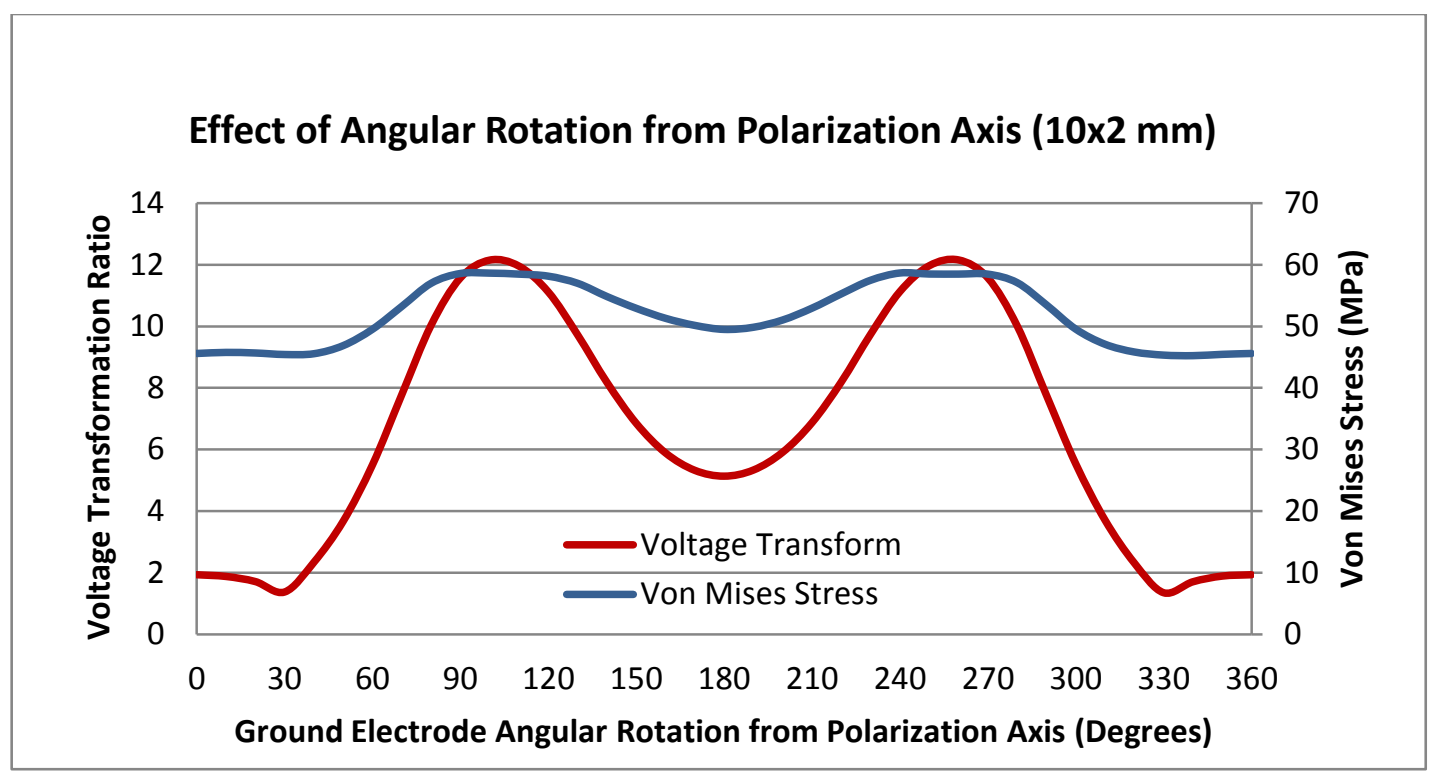

Figure 3.13: Effect of ground plate aperture rotation across surface of disc $(10 \mathrm{x} 2 \mathrm{~mm}$ crystal)

The single frequency off-axis rotation results confirm previous experiments documenting the effect of a rotated polarization axis [25]. Displacement does not occur radially symmetric to the center of the disc because of the $45^{\circ}$ rotated axis, despite being operated at a radial resonance. An offset in the direction of the largest displacement was needed to optimize the voltage transformation according to the constitutive piezoelectric equations (Equations 2.3 and 2.4). Rotating the ground aperture away from the large mechanical displacements should result in a decreased voltage output as less of the aperture is centered along these points. A bench top experiment validated this trend as shown in Figure 3.14. Here, the $\sim 260^{\circ}$ rotation provided an intense bright discharge, while the $\sim 100^{\circ}$ rotation provided a more diffuse glow. Exact placement of the ground aperture was approximated visually. The same experiment on the reverse of the crystal resulted in reversed results. 


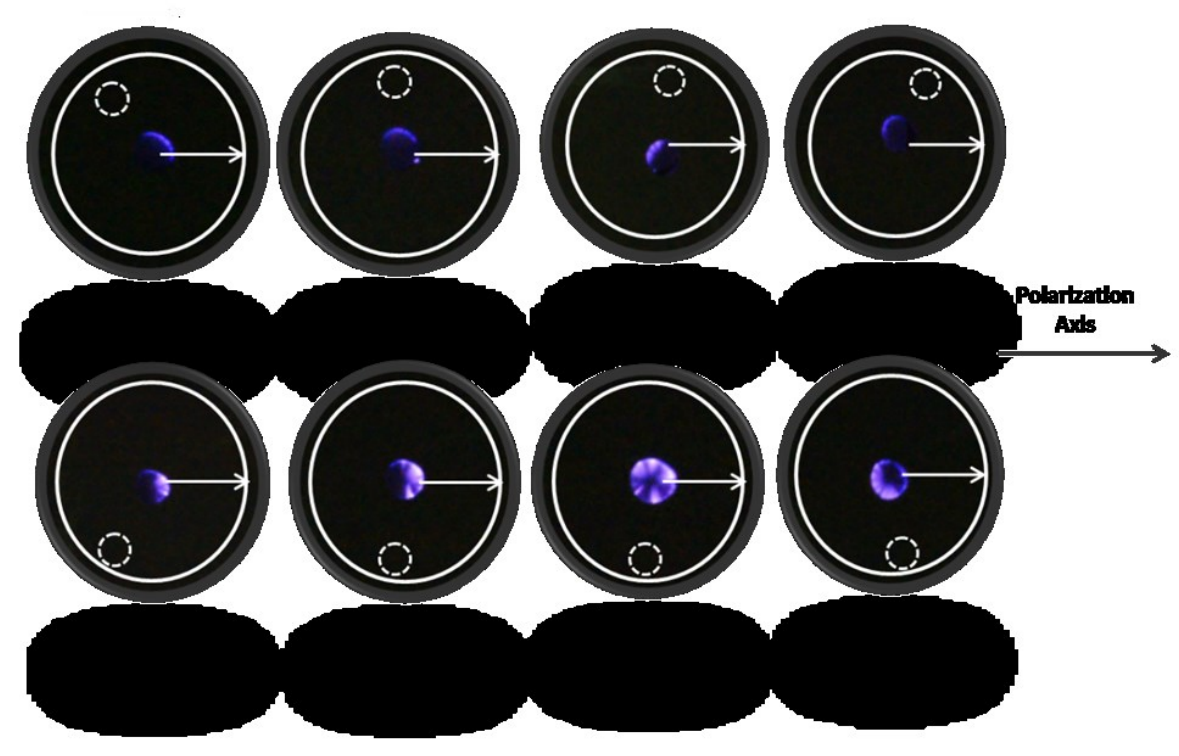

Figure 3.14: Effect of rotating aperture off polarization axis with single resonant frequency of $385 \mathrm{kHz}$. (a) $130^{\circ}$, (b) $100^{\circ}$, (c) $80^{\circ}$, (d) $70^{\circ}$, (e) $250^{\circ}$, (f) $255^{\circ}$, (g) $260^{\circ}$, (h) $280^{\circ}$

A cross sectional plot of the lithium niobate summarizing displacement, potential and stress for aperture placements along the polarization axis, optimal $100^{\circ}$ rotation, and $180^{\circ}$ off of the polarization axis are provided in Figure 3.15 for comparison. 


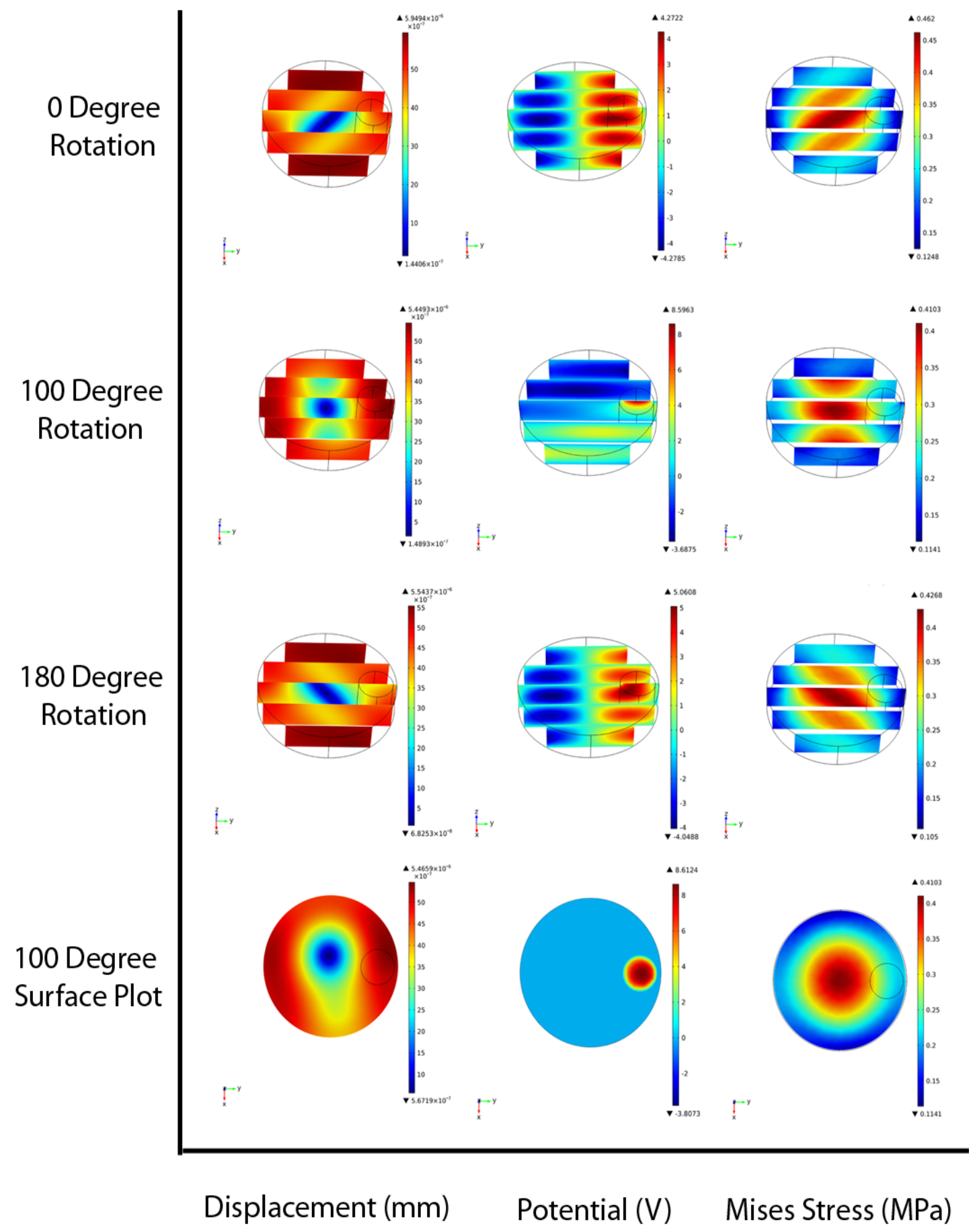

Figure 3.15: Rotational plot comparing displacement, potential and stress. 


\subsubsection{Effect of Crystal Thickness}

The last simulation looked at the effects of varying the crystal thickness to determine if a reduction in von Mises Stress could be achieved. It was noted during experimentation that a number of crystals were experiencing fracture when operated at high duty cycles or voltages. It was suspected that this was a result of high stresses being induced in the crystal. Simulation of the crystal showed the center to exhibit the highest von Mises stress when operated in radial resonance, as shown in Figure 3.16a. This stress pattern corresponded to the majority of crystal fractures travelling through the center of the disc, as shown in Figure 3.16b. High voltages and duty cycles, however, provided the highest ion current used in the generation of thrust. Therefore it was desirable to look at changes to the crystal dimensions to see if any reduction in stress could be obtained while still maintaining the same amount of ion current during operation.
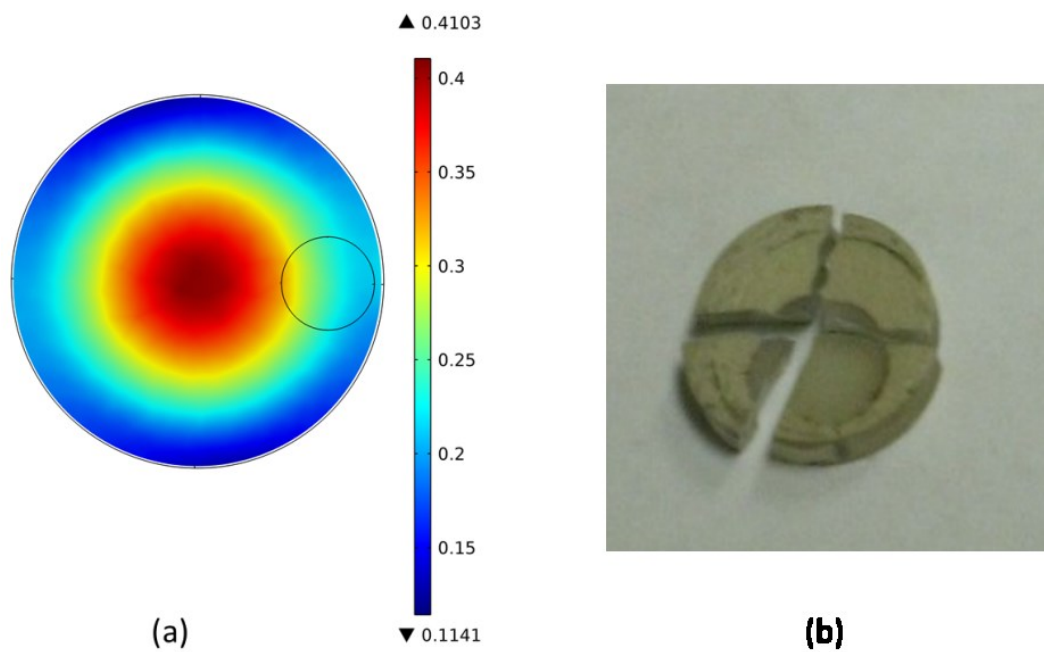

(b)

Figure 3.16: (a) Von Mises stress plot showing highest stress in center of disc.

(b) Crystal failure with fracture through center of disk 
Simulation of the $10 \mathrm{~mm}$ diameter crystal showed a proportional relationship between stress and voltage transformation, with the largest stress and voltage output occurring at a thickness of $2 \mathrm{~mm}$. The high transformation quickly decreased as the thickness increased by as little as $1 \mathrm{~mm}$. All thicknesses beyond this point exhibited no gain in the voltage output.

For the $20 \mathrm{~mm}$ diameter crystal, the maximum transformation again occurred at a thickness of $2 \mathrm{~mm}$. Here, the maximum transformation ratio nearly doubled for nearly the same stress as the $10 \mathrm{~mm}$ crystal. A $50 \%$ reduction of the von Mises stress could be achieved by doubling the thickness to $4 \mathrm{~mm}$, which still maintained the peak voltage transformation found in the $10 \mathrm{~mm}$ crystal. Therefore, using a larger crystal could be a desirable method to either maintain the same level of ion current while reducing the stress, or it could be used to decrease the input voltages required by increasing the step up voltage transformation, without increasing the von Mises stress found in the smaller crystal. 


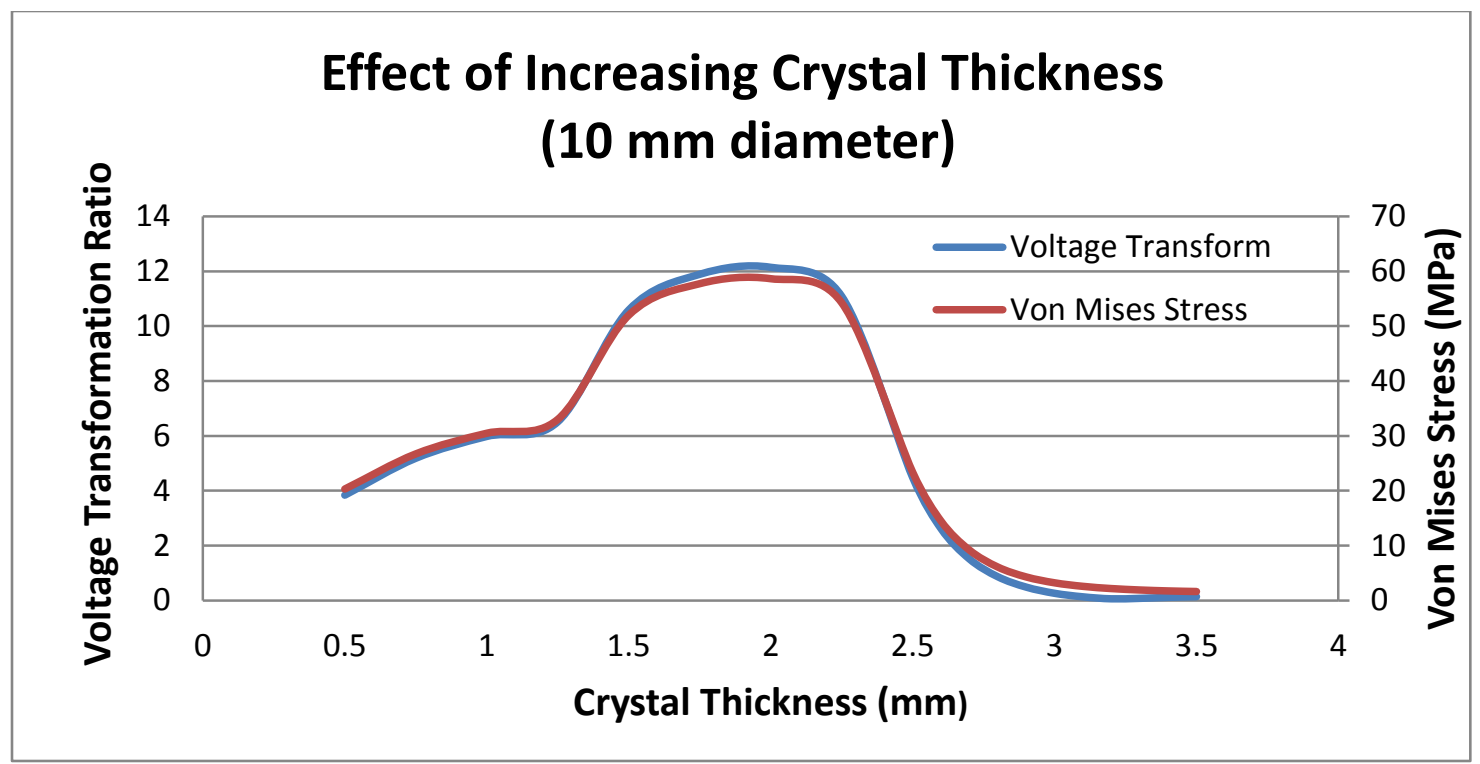

Figure 3.17: Stress and voltage transformation corresponding to increasing crystal thickness for a $10 \mathrm{~mm}$ diameter crystal and $100 \mathrm{~V}$ applied voltage.

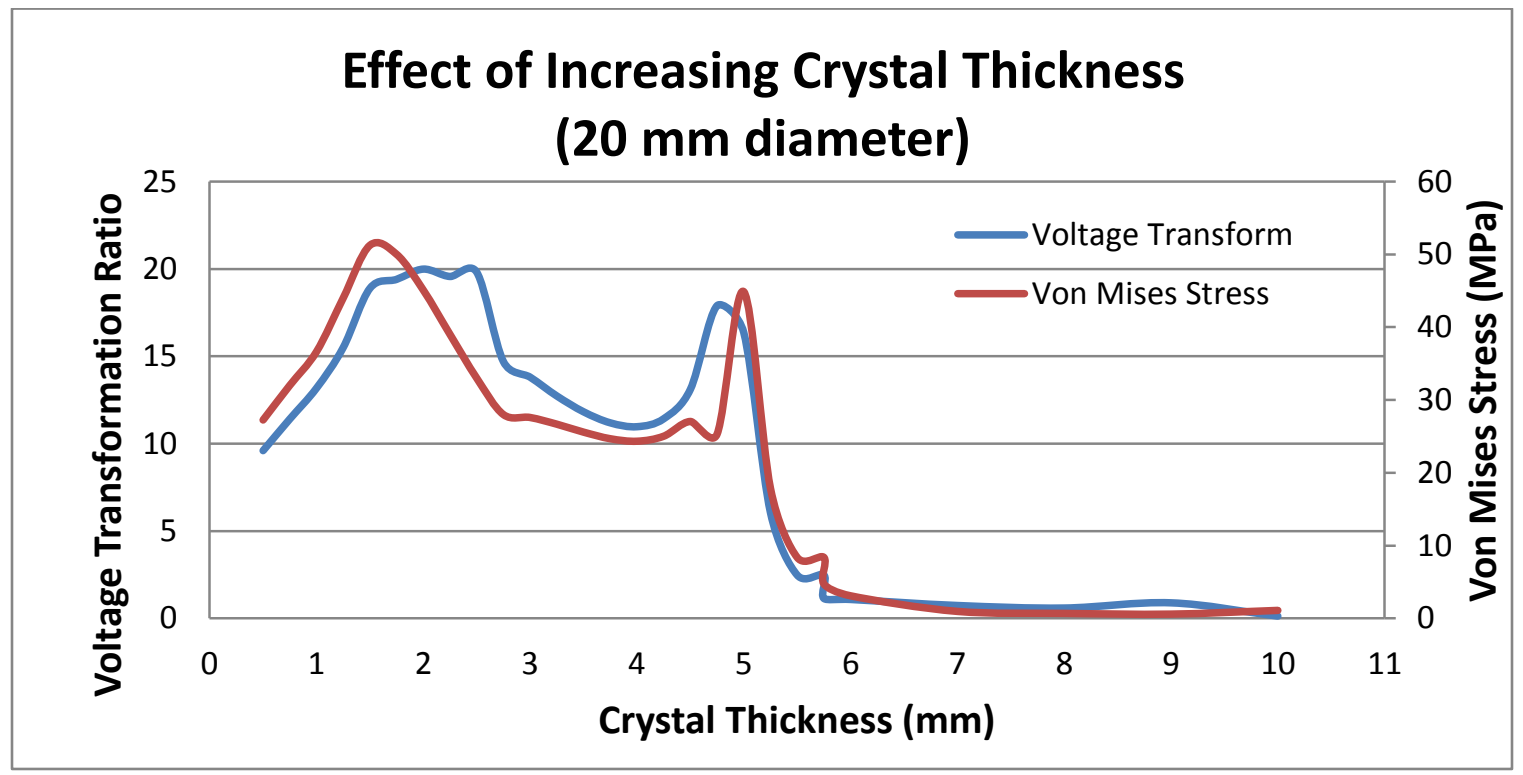

Figure 3.18: Stress and voltage transformation corresponding to increasing crystal thickness for a $20 \mathrm{~mm}$ diameter crystal and $100 \mathrm{~V}$ applied voltage. 


\subsection{Conclusion}

Simulation of the lithium niobate crystal helped refine lab procedures used to prepare and operate the PTPS-MT. Bench top experiments were used to confirm results found in the simulation models, improving the performance over previously documented parameters [25]. A summary of crystal and electrode parameters used in testing are shown in Table 3.3. To ensure uniformity between experiments requiring a change of the crystal, all preparation was done according to procedures found in Appendix A: Crystal Preparation. Final operation of the PTPS-MT required slight tensioning adjustments to match the frequency between experiments, as described in section 3.1.2, to account for the physical clamping caused by the ground electrode in the old holder design.

Table 3.3: Final crystal design parameters

\begin{tabular}{|l|l|}
\hline Parameter & Value \\
\hline Radial Offset & $70 \%$ Crystal Radius \\
\hline Angular Rotation & $+/-100^{\circ}$ off polarization axis \\
\hline $\begin{array}{l}\text { Ground Electrode Aperture } \\
\text { Diameter }\end{array}$ & $25 \%$ Crystal Radius \\
\hline Crystal Thickness & $2 \mathrm{~mm}$ \\
\hline \multirow{2}{*}{ Resonant Frequency } & $10 \mathrm{~mm}=396 \mathrm{kHz}^{*}$ \\
\cline { 2 - 2 } & $20 \mathrm{~mm}=199 \mathrm{kHz}^{*}$ \\
\hline
\end{tabular}

${ }^{*}$ Approximate. Effect of physical clamping requires adjustment. 


\section{Chapter 4}

\section{PTPS-MT Thruster Analysis}

This chapter will cover the results and analysis of all experiments done during the operation and refinement of the PTPS-MT. Results will be presented beginning with the changes to operational parameters on emitted current, followed by overall performance and reliability of the thruster. All experiments were performed in a vacuum chamber with base pressures of $10^{-5}-10^{-6}$ Torr with a locally high pressure created near the surface of the lithium niobate to aid in plasma formation.

\subsection{Measurement of Ion Current}

\subsubsection{Effect of Aperture Diameter on Collected Current}

The first set of experiments looked at the effect of the diameter of the containment aperture seen in Figure 4.1. Work by Hutsel used apertures ranging from $2 \mathrm{~mm}$ up to $8 \mathrm{~mm}$ in an attempt to partially contain a propellant gas [25]. These designs suffered from high mass flows needed to obtain a gas pressure capable of sustaining a plasma discharge. The result was inefficient propellant use and low specific impulses. The diameters used for the PTPS-MT ranged from $0.3 \mathrm{~mm}$ to $0.9 \mathrm{~mm}$ with the expectation that flow rates of propellant gas could be better contained for ionization, thereby increasing the utilization efficiency. Measurements for ion current were conducted with three gases: hydrogen, nitrogen, and argon. Typically use of $\mathrm{H}_{2}$ and $\mathrm{N}_{2}$ gas is undesirable in ion thrusters due to the low molecular weight resulting in low kinetic energy used to 
generate thrust. They were included in this research for comparison and to support other research efforts. Results are broken down by gas and aperture diameter used.

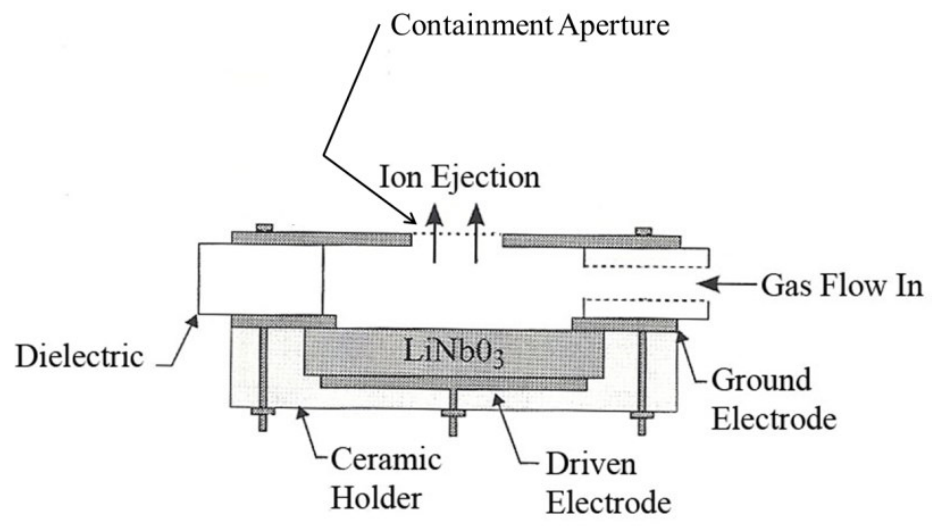

Figure 4.1: Physical model of PTPS-MT with containment aperture used to limit neutral flow losses to vacuum.

Measurement of emission current was done with the charge collecting plate described in Section 2.3.1. The gas flow was incremented using a Celerity 7300 mass flow controller with maximum flow rates of $2.82 \times 10^{-6} \mathrm{~kg} / \mathrm{s}(42 \mathrm{sccm})$ for argon, $1.9 \times 10^{-6} \mathrm{~kg} / \mathrm{s}(30 \mathrm{sccm})$ for nitrogen, and $1.4 \times 10^{-7} \mathrm{~kg} / \mathrm{s}(30.3 \mathrm{sccm})$ for hydrogen. An applied RF voltage amplitude of $1190 \mathrm{~V}$ was used to provide a consistent result for comparison. Use of lower amplitudes did not always provide a reliable plasma formation at lower mass flow rates. A small negative DC bias of $50 \mathrm{~V}$ was applied to the charge collecting plate to shield out any electron current, allowing for comparisons of only ion current. Without this small applied bias voltage, the slow speed of the ions would result in ion current being collected at the same time as emission of electron current from subsequent RF cycles were reaching the plate, resulting in an overall negative current being collected. 
The use of larger apertures increased the measured peak ion current for all three gasses. For hydrogen, use of the $0.6 \mathrm{~mm}$ aperture provided three times the $0.3 \mathrm{~mm}$ aperture current, as shown in Figure 4.2. The $0.9 \mathrm{~mm}$ aperture failed to produce reliable plasma even at the highest mass flow rate available $(30.3 \mathrm{sccm})$ on the mass flow controller (MFC) and was not included in the analysis. Occasional discharges would occur, most likely after a buildup of enough propellant within the chamber to create plasma occurred.

Figure 4.3 showed nitrogen followed a similar trend, increasing collected ion current with each increase in aperture diameter. Peak ion current for hydrogen and nitrogen were nearly identical. The mass flow rate for nitrogen, however, was over three times the flow rate of hydrogen for the same current output.

For argon, the emitted ion current remained low until use of the $0.9 \mathrm{~mm}$ aperture. Figure 4.4 shows a significant increase of measured ion current on the charge collecting plate. Compared to nitrogen and hydrogen, the measured current for argon with the $0.9 \mathrm{~mm}$ aperture was the largest of all three gases. This is favorable for an ion thruster as both the large ion current and large mass are associated with the same gas. This would result in the largest kinetic energy transfer of the three available options. 


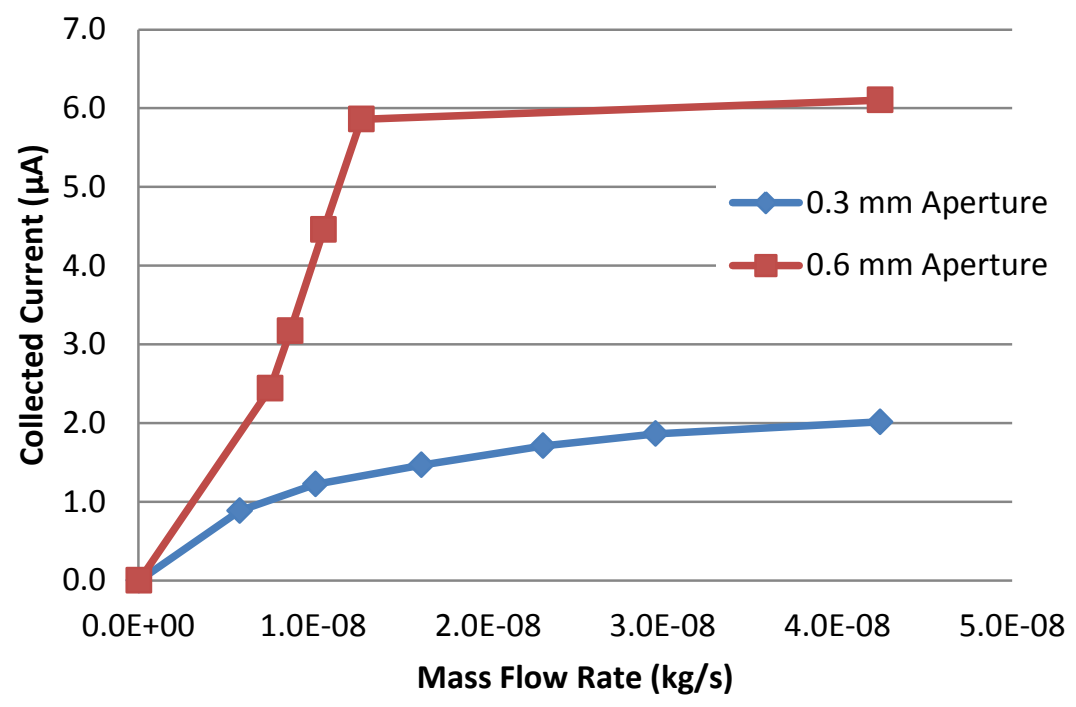

Figure 4.2: Collected emission current for hydrogen propellant. Use of the $0.9 \mathrm{~mm}$ aperture not included due to inability to form plasma at available flow rates.

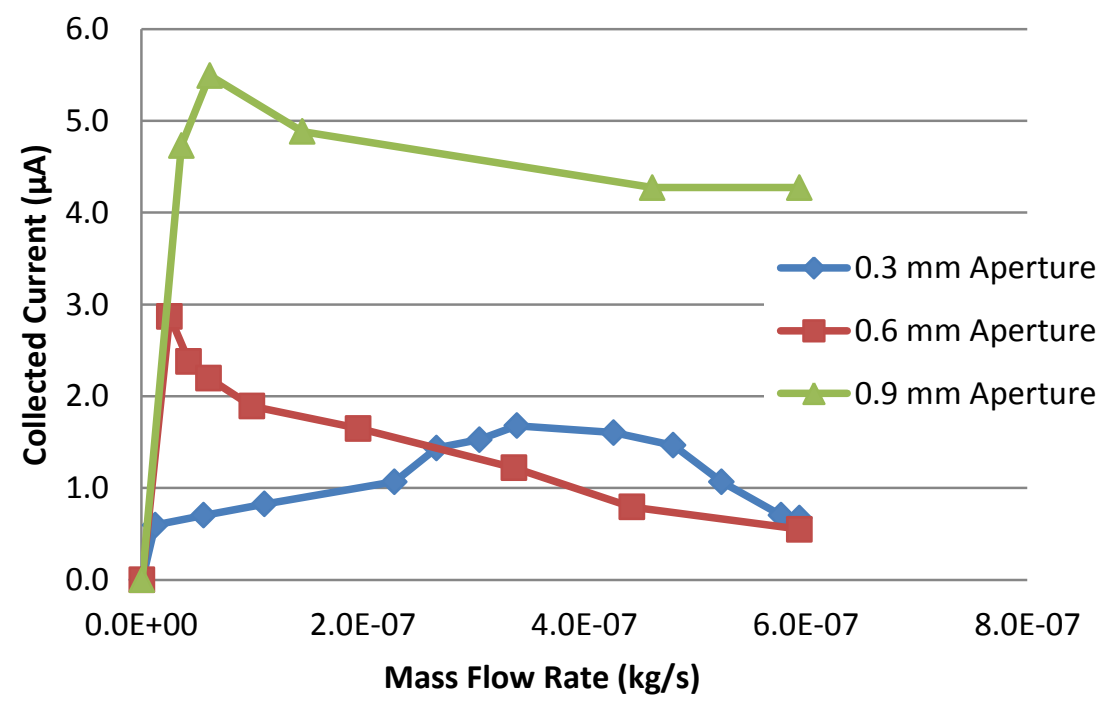

Figure 4.3: Collected ion current for nitrogen propellant at three selected aperture diameters 


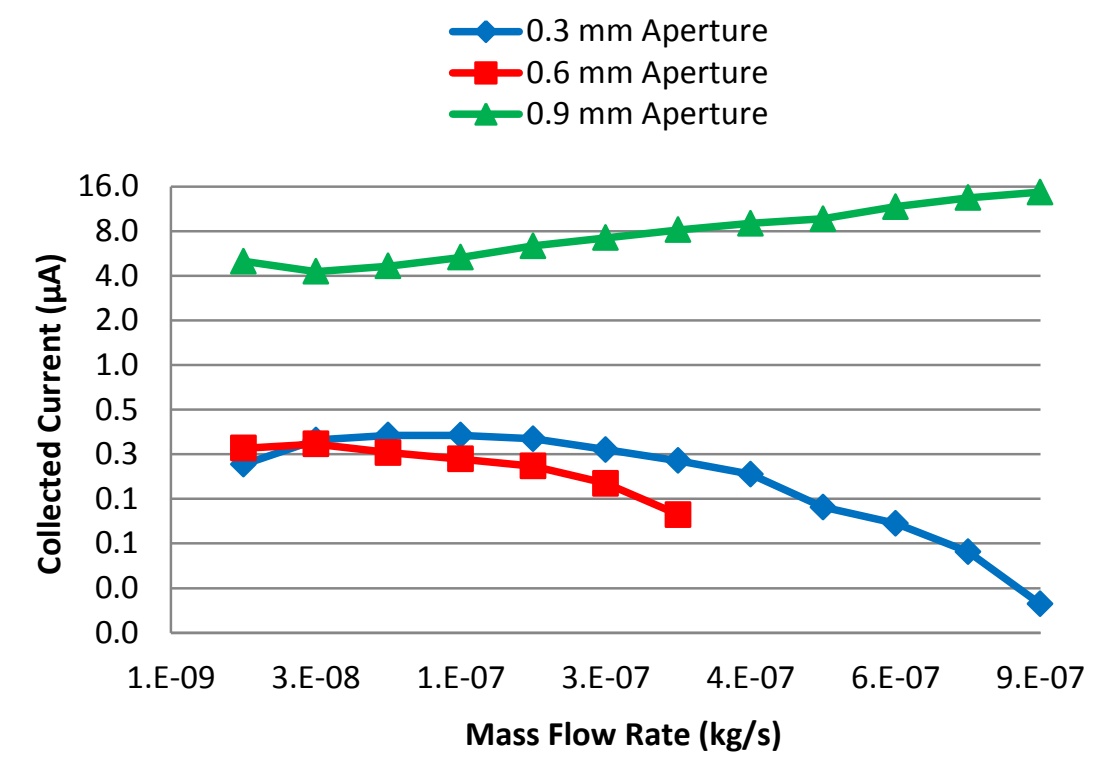

Figure 4.4: Collected ion current for argon propellant at three selected aperture diameters

In all cases, the ion current increased with an increase in the containment aperture diameter. This trend corresponds to the electric current density, where the measured plate current would increase with an increase in aperture area, according to:

$$
I=J A=q n_{\text {plasma }} v_{\text {bohm }} A
$$

where it is assumed that the plasma density, $n_{\text {plasma }}$, and Bohm velocity, $v_{\text {bohm }}$, remain unchanged.

\subsubsection{Effect of Driving Voltage on Collected Current}

The effect of varying input voltage to the PTPS-MT was investigated to determine if any significant increase in voltage would produce an increase in the collected ion current. If no significant increase was present, minimizing the power supply used to drive 
the thruster would allow for reduced weight on an overall rocket propulsion system. Measurement of ion current was conducted at an aperture diameter of $0.6 \mathrm{~mm}$ for all three gasses, with flow rates again incremented from zero up to the maximum mass flow available from the MFC. A small DC voltage of $50 \mathrm{~V}$ was again applied to the charge collecting plate to limit the collection of electron current.

For hydrogen, significant flow rates were required before the formation of a plasma would occur at the lower voltages. Figure 4.5 shows only in the case of the highest applied voltage a breakdown able to occur at lower flow rates. This is likely due to the higher energy of electrons, from the increased driving voltage, providing an increase in the amount of ionization. Hydrogen also provided the largest amount of collected current of the three tested gasses.

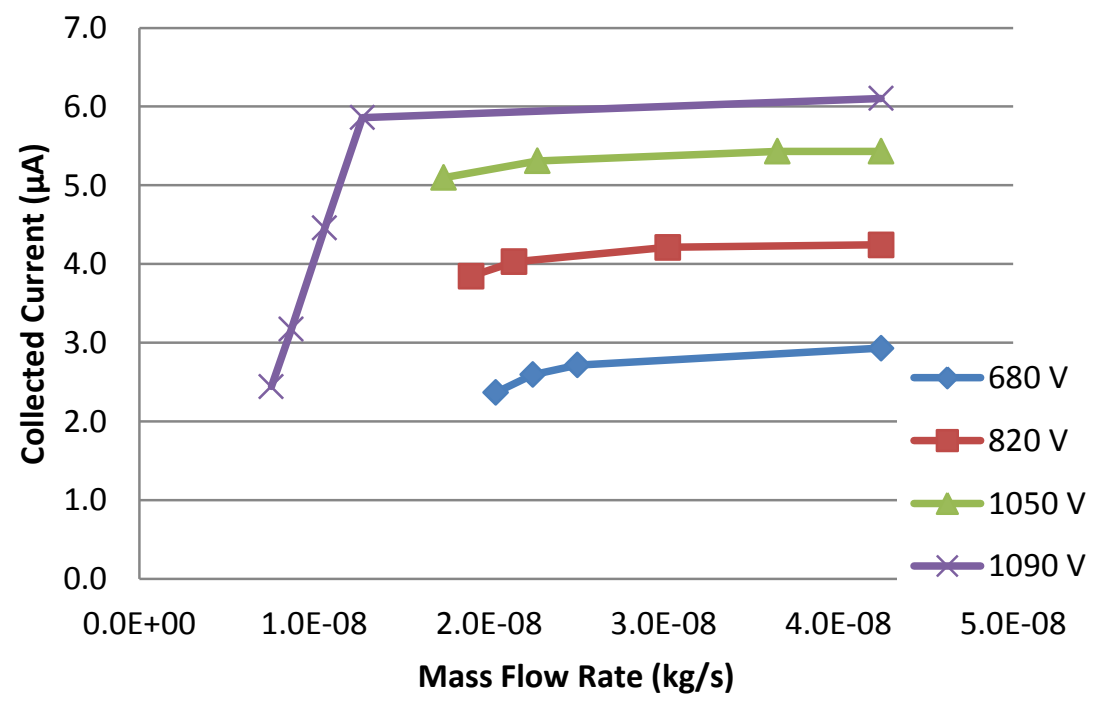

Figure 4.5: Collected ion current for hydrogen with four increasing applied voltages. Increasing plasma density occurs with an increase in driving voltage. 


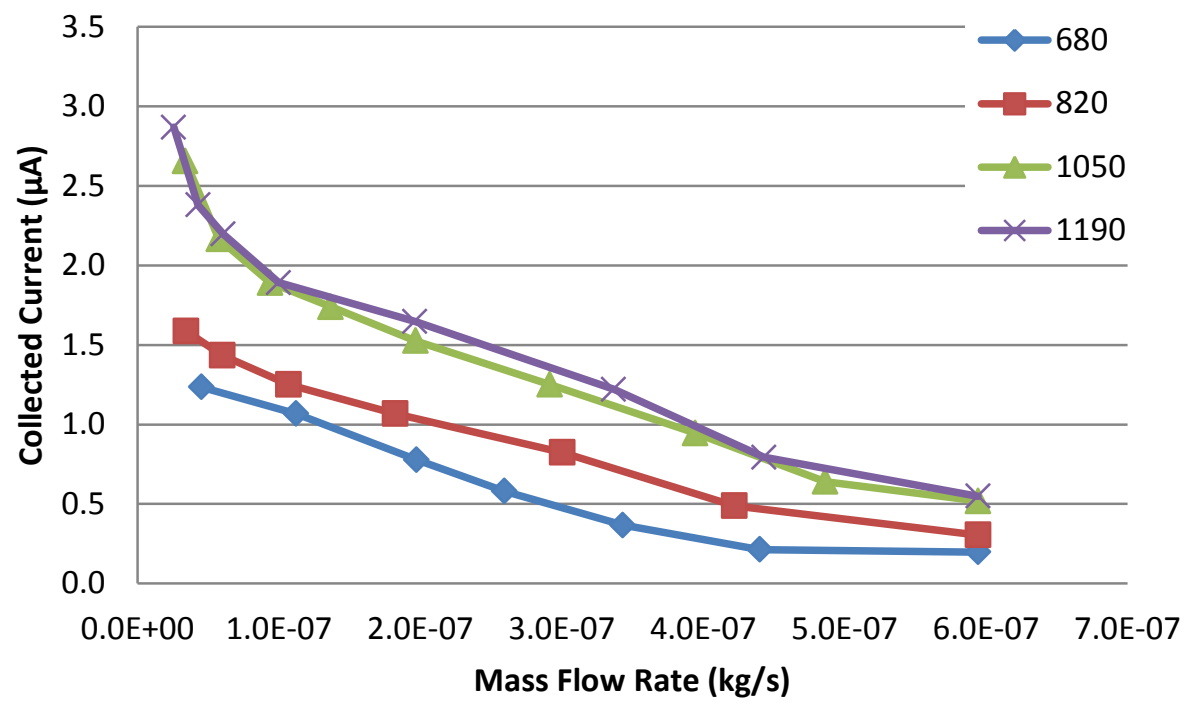

Figure 4.6: Collected ion current for nitrogen with four increasing applied voltages.

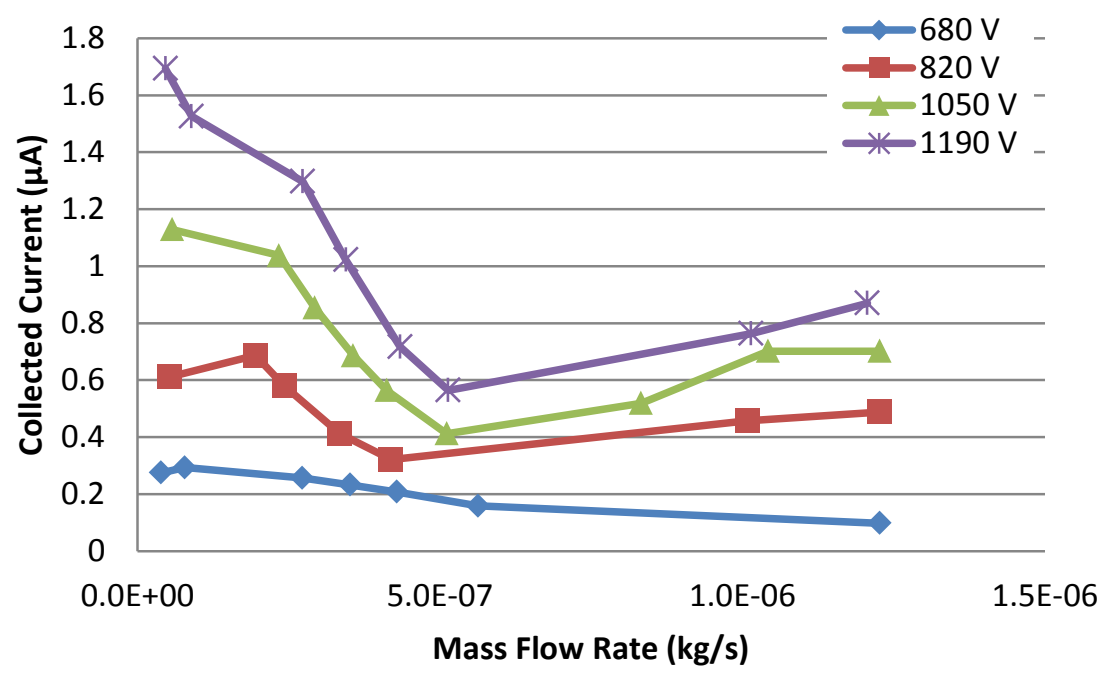

Figure 4.7: Collected ion current for argon with four increasing applied voltages. 
Results from increasing the applied voltage show a significant increase in peak ion current. As gas flow is increased, the plasma density decreased, a trend also shown with nitrogen in Figure 4.6. At higher flow rates, argon experienced an increase in the collected current. It was noted at these points that discharges outside the plasma chamber were beginning to occur, resulting in the increased collected ion current. Similar external discharges did not occur for nitrogen or hydrogen.

\subsubsection{Effect of Extraction Voltage on Collected Current}

Changes to the voltage applied to the charge collecting plate were conducted to simulate the effect of having an acceleration grid found on an electrostatic ion thruster. Acceleration grids are used to increase the velocity of ejected ions resulting in increased thrust. They also aid thruster operation by drawing a larger portion of ions out of the ionization chamber and containing electrons, which further aid in ionization of neutrals [4]. The drawback of having an acceleration grid is the need for a separate power source resulting in increased complexity and cost. A separate beam neutralization system would also be required as only ions would be selectively emitted resulting in a negative charge being developed on the spacecraft which could cause a catastrophic failure for onboard components.

Three voltages were applied to the charge collection plate: $0 \mathrm{~V}, 50 \mathrm{~V}$, and $1140 \mathrm{~V}$. The $0 \mathrm{~V}$ was selected to determine if any fields generated by the piezoelectric crystal would provide suitable ion current without requiring additional components to generate thrust. The $50 \mathrm{~V}$ application was selected as a low voltage to screen any electron current with the intent that a small acceleration grid could be made with negligible overhead in 
the power requirements for an appropriate design. The last applied voltage was used as an attempt to draw the largest amount of ion current in order to determine peak operation of the PTPS-MT if a gridded accelerator design was approached. For both the 50 and 1140 $\mathrm{V}$ applications, use of a secondary screening grid was used to prevent false ion current readings resulting from the loss of secondary electrons on the charge collecting plate.

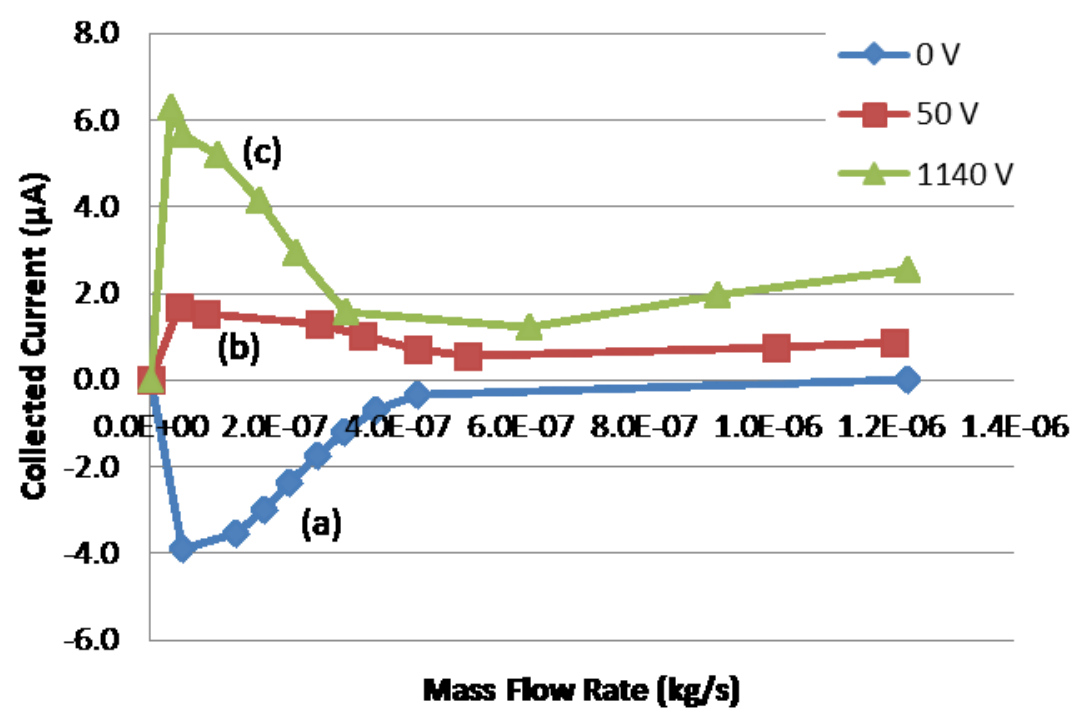

Figure 4.8: Collected argon current with three applied extraction voltages. Three effects are noted (a) high electron mobility resulting in collection of negative charge, (b) ion extraction, (c) high plasma density extraction 


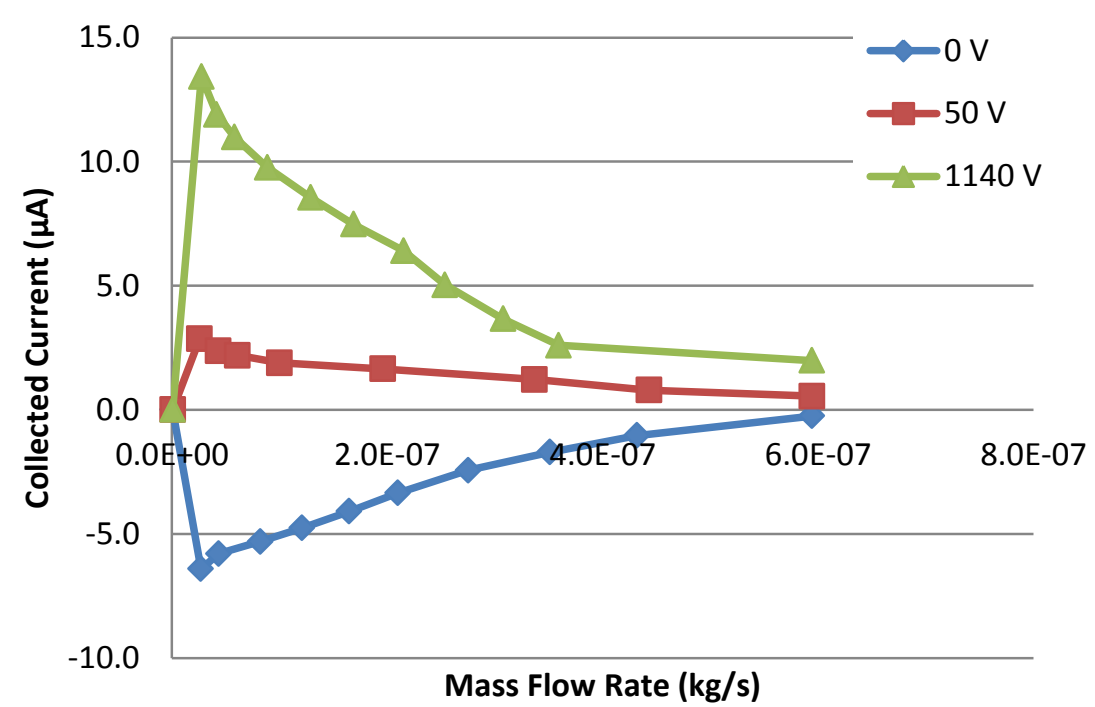

Figure 4.9: Collected nitrogen current with three applied extraction voltages.

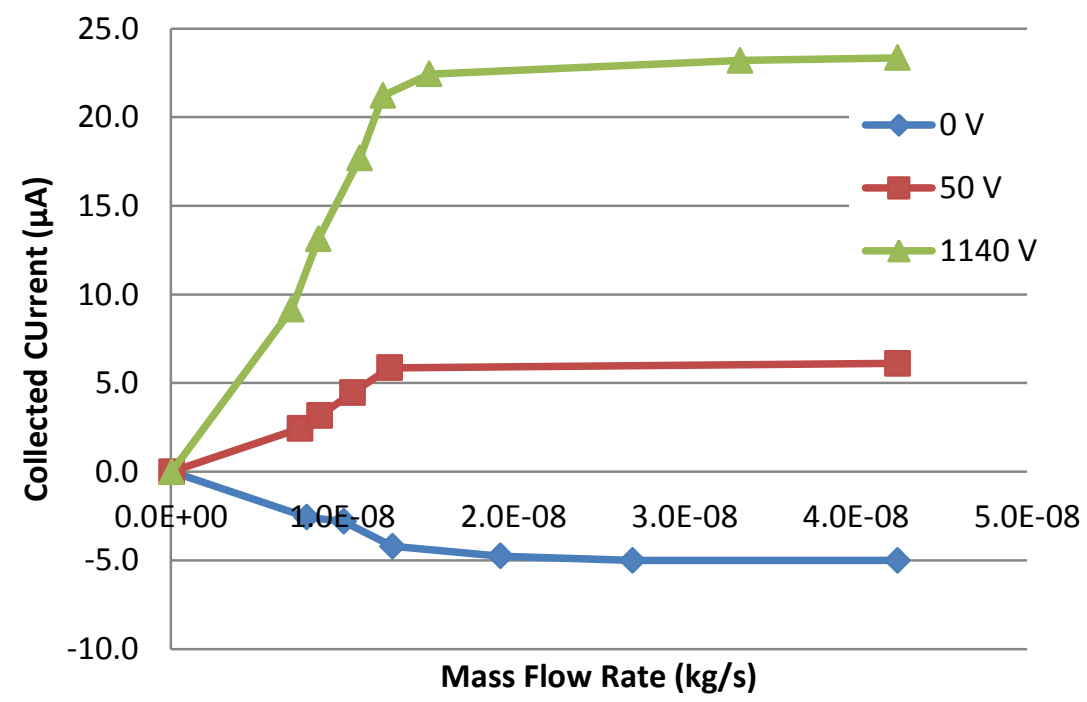

Figure 4.10: Collected hydrogen current with three applied extraction voltages.

As expected for the $0 \mathrm{~V}$ potential, a collection of negative current occurred for all three gasses. This occurred as a result of high electron mobility resulting in subsequent $\mathrm{RF}$ cycle electron emission to reach the measurement plate in the same period as earlier 
emitted ions. The continuing cycle of discharges in the RF burst would negate ion readings without any form of electron screening.

A small DC voltage of $50 \mathrm{~V}$ was enough to shield electron current resulting in small extracted ion current readings.

Application of a large accelerating voltage showed a significant increase in collected current, suggesting that if the PTPS-MT was to be used as a thruster, designs incorporating an acceleration grid would likely be desired.

\subsection{Thrust Calculations}

\subsubsection{Mass Flow Utilization}

Thrust in a rocket system is given by:

$$
T=\frac{d m_{p}}{d t} v_{e x} \approx \dot{m}_{\iota} v_{i} \quad[\mathrm{~N}]
$$

where $m_{i}$ is the mass flow rate of the exhaust and $v_{i}$ is the speed of the exhaust gas measured relative to the rocket [4]. In an ion thruster, the total thrust is related to the ability to ionize the background gas to generate ions. It is unlikely that complete ionization would ever occur, resulting in a beam consisting of both ions and neutral background gas. The ions have a high effective velocity due to electrostatic acceleration at the PTPS-MT exit. The neutrals have a low effective velocity attributed to their thermal energy. The resulting thrust is therefore the sum of both the ion thrust and neutral gas thrust: 


$$
T_{\text {total }}=T_{\text {ion }}+T_{\text {neutral }}
$$

To achieve a high total thrust with a high propellant utilization, designs that limit the flow of neutrals while simultaneously increasing ionization are desired to have an effective system for space propulsion.

Current measurements from the charge collection plate, described in section 2.3.1, were used to determine the ion mass flow rate:

$$
\dot{m}_{\text {ion }}=\frac{I_{\text {avg }} m_{\text {ion }}}{q} \quad[\mathrm{~kg} / \mathrm{s}]
$$

where $I_{\text {avg }}$ is the average ion current measured on the charge collection plate, $m_{\text {ion }}$ is the ion mass, and $q$ is the fundamental charge constant.

Ion velocity was mathematically calculated according to:

$$
v_{i}=\sqrt{\frac{2 q V_{b}}{m_{i o n}}} \quad[\mathrm{~m} / \mathrm{s}]
$$

where $V_{b}$ is the accelerating potential applied to the ions [31]. For calculations, $V_{b}$ was the sum of the applied voltage to the crystal and the DC extraction voltage. Verification of the calculated speed employed a Faraday cup to measure time of flight as described in Section 2.3.2. With the cup placed at a distance of $5 \mathrm{~cm}$, the transit time was calculated by measuring the time for ion current to appear after the formation of plasma. The resulting velocity was then calculated using:

$$
v_{\text {ion }}=\frac{\Delta x}{\Delta t}
$$


Results for both the mathematical calculation of ion velocity and the Faraday cup agreed to within $10 \%$. These results also confirm ion velocities found by Hutsel where the use of a similar setup calculated ion velocities at $71,164 \mathrm{~m} / \mathrm{s}$ with no external accelerating potential [25]. Due to limitations in using the Faraday cup with other diagnostic equipment, the mathematical approach was used for all other thrust calculations.

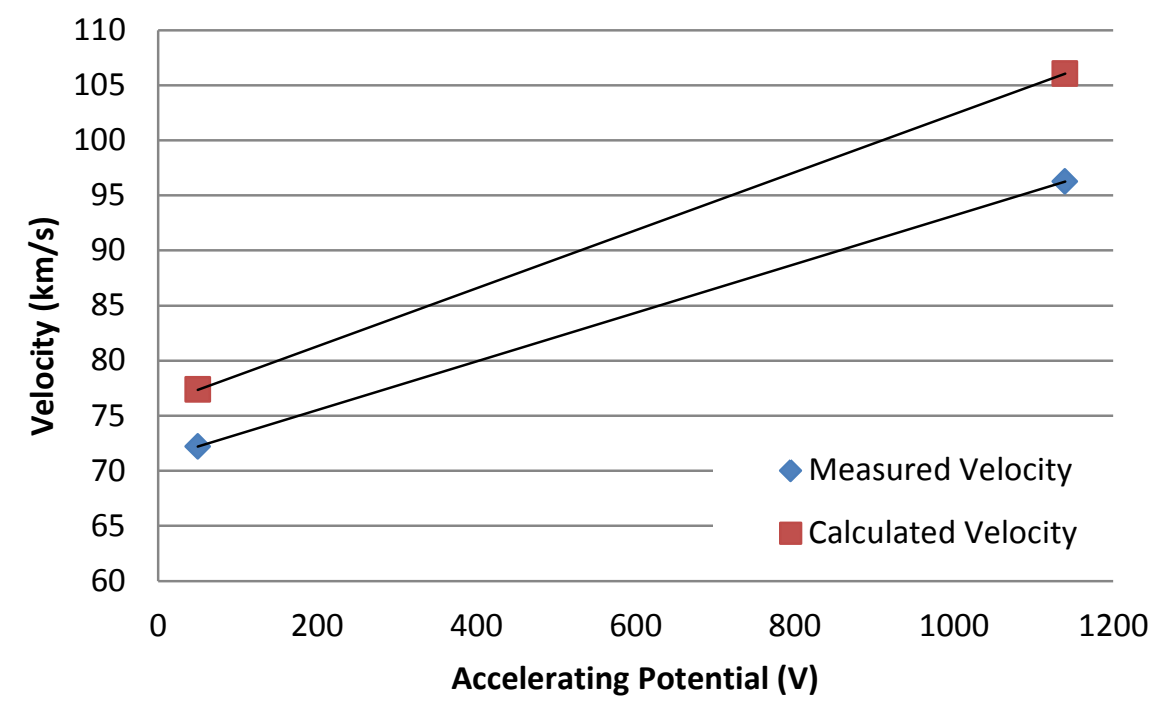

Figure 4.11: Ion velocities for argon under 50 and $1140 \mathrm{~V}$ applied accelerating voltage.

With the velocity, ion energy, $E_{\text {ion }}$, was calculated according to:

$$
E_{i o n}=\frac{1}{2} m_{i o n} v_{i o n}{ }^{2}
$$

The total ion thrust could be found directly by combining equation $4.1,4.3$ and 4.6 to get: 


$$
\left|T_{\text {ion }}\right|=I_{\text {avg }} \sqrt{\frac{2 m_{\text {ion } E}}{q}}
$$

Figure 4.12 shows generated ion thrust for all three gasses. At low flow rates, nitrogen and argon achieved similar thrust with argon increasing by a factor of three at the highest flow rate. As expected, hydrogen generated negligible thrust due to the low mass of the $\mathrm{H}_{2}$ ion despite having the highest measured ion currents.

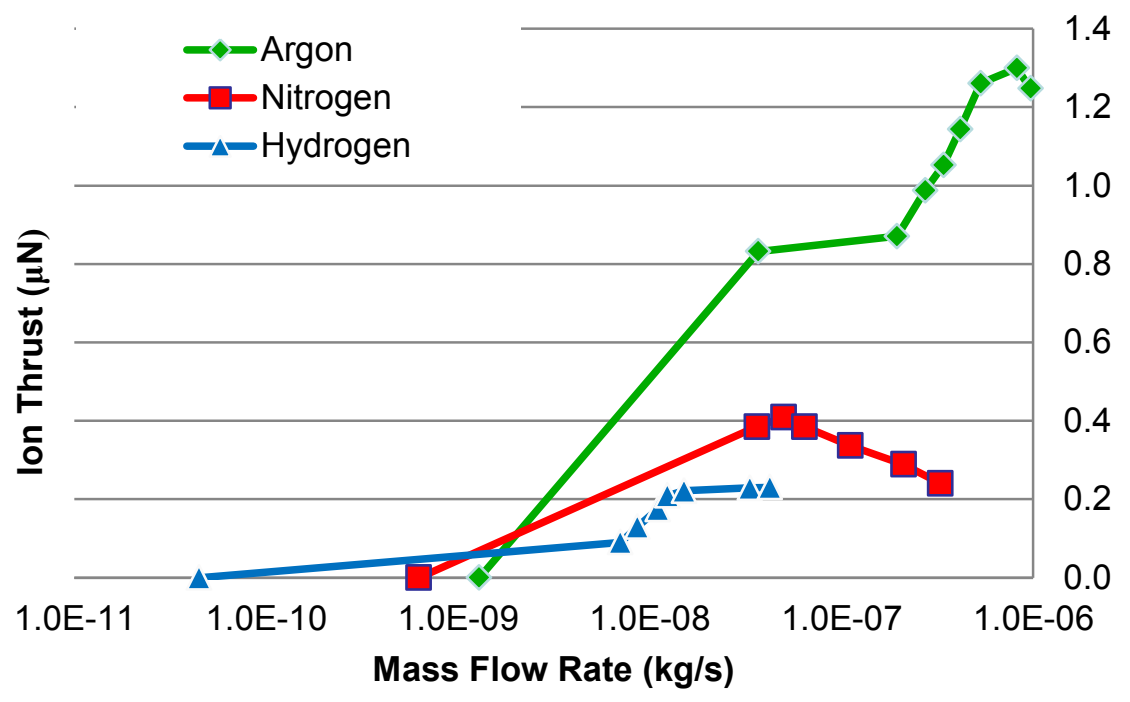

Figure 4.12: Maximum obtained ion component of thrust for each propellant. $1190 \mathrm{~V}$ driving voltage and $1140 \mathrm{~V}$ extraction used for all three gasses. Graph endpoints correspond to maximum flow rate for MFC with selected gas.

\subsubsection{Estimation of Neutral Flow}

To determine the effect of neutral gas flow on total thrust, the neutral mass flow rate and neutral velocity are needed according to Equation 4.1. The neutral mass flow rate 
was found as the difference between the total gas flow rate to the thruster and the ion mass flow rate calculated in Equation 4.11:

$$
\dot{m}_{\text {neutral }}=\dot{m}_{\text {total }}-\dot{m}_{\text {ion }}
$$

The total mass flow rate was measured by the MFC in units of standard cubic centimeters per minute (sccm). Corrections to account for the use of different gas types and conversion to units of $\frac{\mathrm{kg}}{\mathrm{s}}$ were performed and are found in Appendix B. The total mass flow rate is given by:

$$
\dot{m}_{\text {total }}=\frac{m_{\text {neutral }} Q}{k T}
$$

Where $Q$ is the mass flow rate provided by the MFC, $k$ is Boltzmann's constant, and $T$ is the gas temperature in Kelvin. The gas temperature was assumed to be at $20^{\circ} \mathrm{C}$. The average neutral velocity in thermal equilibrium can be found by taking the average of a Maxwellian distribution to obtain:

$$
\bar{v}_{\text {neutral }}=\sqrt{\left(\frac{8 k T}{\pi m}\right)}
$$

The thermal velocities for experimental gasses are provided in Table 4.2:

Table 4.2: Thermal velocities of PTPS-MT gasses.

\begin{tabular}{|l|l|}
\hline Ion & Velocity \\
\hline Argon & $380 \mathrm{~m} / \mathrm{s}$ \\
\hline Nitrogen & $454 \mathrm{~m} / \mathrm{s}$ \\
\hline Hydrogen & $1,693 \mathrm{~m} / \mathrm{s}$ \\
\hline Electron & $94,786 \mathrm{~m} / \mathrm{s}$ \\
\hline
\end{tabular}


The total thrust generated by the neutral gas flow is then found by combining Equation 4.10 with 4.9 to obtain:

$$
T_{\text {neutral }}=\dot{m}_{\text {neutral }} \sqrt{\left(\frac{8 k T}{\pi m}\right)}
$$

The total thrust of the PTPS-MT is a simple sum of the neutral and ion thrust:

$$
T_{\text {ion }}+T_{\text {neutral }}=T_{\text {total }}
$$

In Figure 4.13, the axis on the left represents the ion thrust for three aperture sizes of argon. For all three cases, the neutral flow dominated resulting in the linear increase in neutral thrust proportional to the increase in mass flow rate. The thrust from the neutral flow was more than 4 orders of magnitude larger, making any effect due to ion thrust negligible.

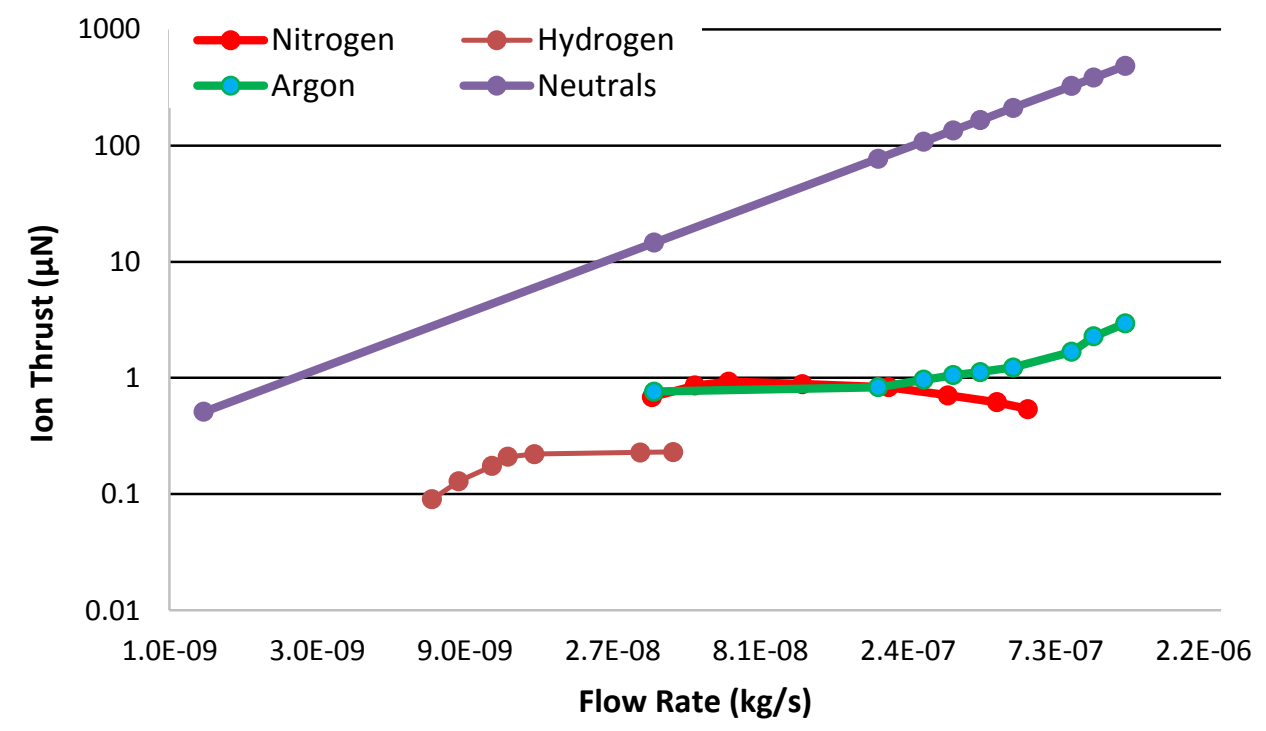

Figure 4.13: Left axis shows ion thrust for argon, while the straight line and right axis represents neutral thrust. 


\subsubsection{Specific Impulse}

Thruster efficiency is often measured by the specific impulse, $I_{s p}$ [4]. Specific impulse is the ratio of thrust generated per mass of propellant used per unit time:

$$
I_{s p}=\frac{T_{\text {total }}}{\dot{m}_{\text {total }} \cdot g_{0}}[\mathrm{~s}]
$$

where $g_{0}$ is the gravitational constant. The specific impulse of the PTPS-MT was computed for a range of flow rates and input voltages with results shown in Figure 4.14. The PTPS-MT thrust was dominated by the neutral flow. Therefore, the specific impulse only depended on the gas flow rate, resulting in a constant value across all testing parameters. For direct comparison, many present day ion propulsion systems have a specific impulse of around 10,000 s.

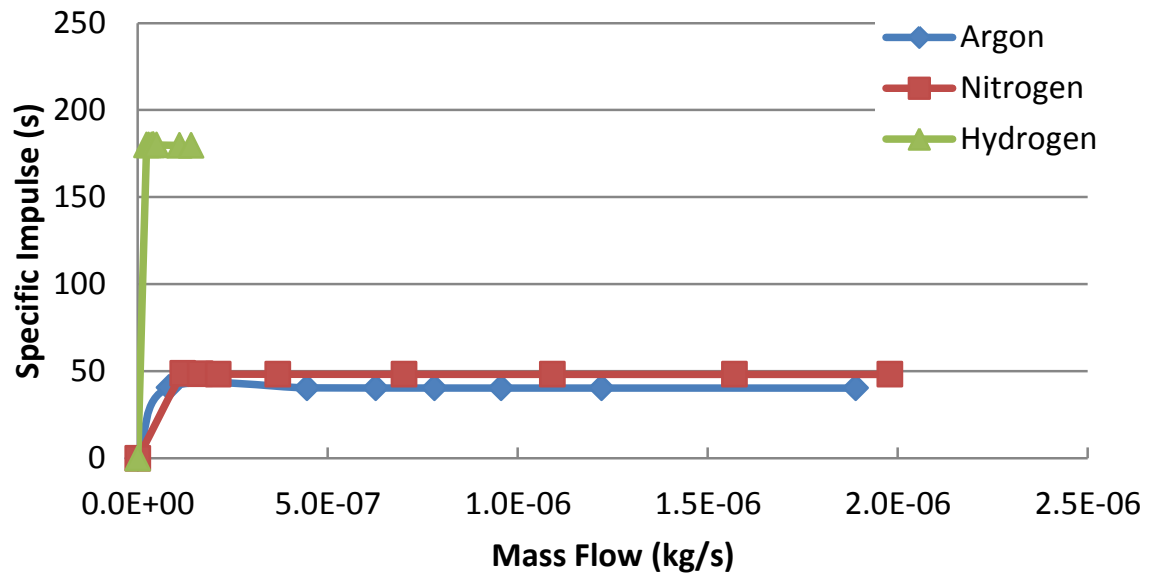

Figure 4.14: Specific impulse of PTPS-MT for three selected gasses. 
The specific impulse found in the PTPS-MT is extremely low as a result of the high neutral flow. Argon and nitrogen have specific impulses of around $45 \mathrm{~s}$, while hydrogen achieved around $175 \mathrm{~s}$.

\subsubsection{Thruster Efficiency}

The efficiency of the PTPS-MT relies on a large portion of the input power being converted into ion thrust:

$$
\eta=\frac{\frac{1}{2} T_{i o n} v_{i o n}}{P_{\text {avg }}} * 100
$$

where $\eta$ is the overall efficiency and $P_{a v g}$ is the average RMS power delivered to the thruster. Because of the low ion thrust experienced, overall efficiencies were expected to be less than $2 \%$, as shown in Table 4.3 .

Table 4.3: Selected efficiencies for argon at typical input powers

\begin{tabular}{|l|l|}
\hline Input Power (W) & Efficiency (\%) \\
\hline 5.04 & 1.10 \\
\hline 6.41 & 1.27 \\
\hline 7.58 & 1.24 \\
\hline 10.36 & 0.94 \\
\hline
\end{tabular}

If the thruster were able to deliver a larger percentage of ion thrust, efficiencies could improve significantly. 


\subsubsection{Floating Voltage}

The thruster assembly was electrically isolated from ground potential to experimentally show the effects of charge buildup due to the loss of ions or electrons. A small positive charge buildup would be considered acceptable provided no discharges occurred which could have a detrimental effect on onboard electronic equipment. For an extraction voltage of $0 \mathrm{~V}$, results of the charge buildup on the outer aperture are shown in Figure 4.15 (refer to Figure 2.4 for experimental illustration).

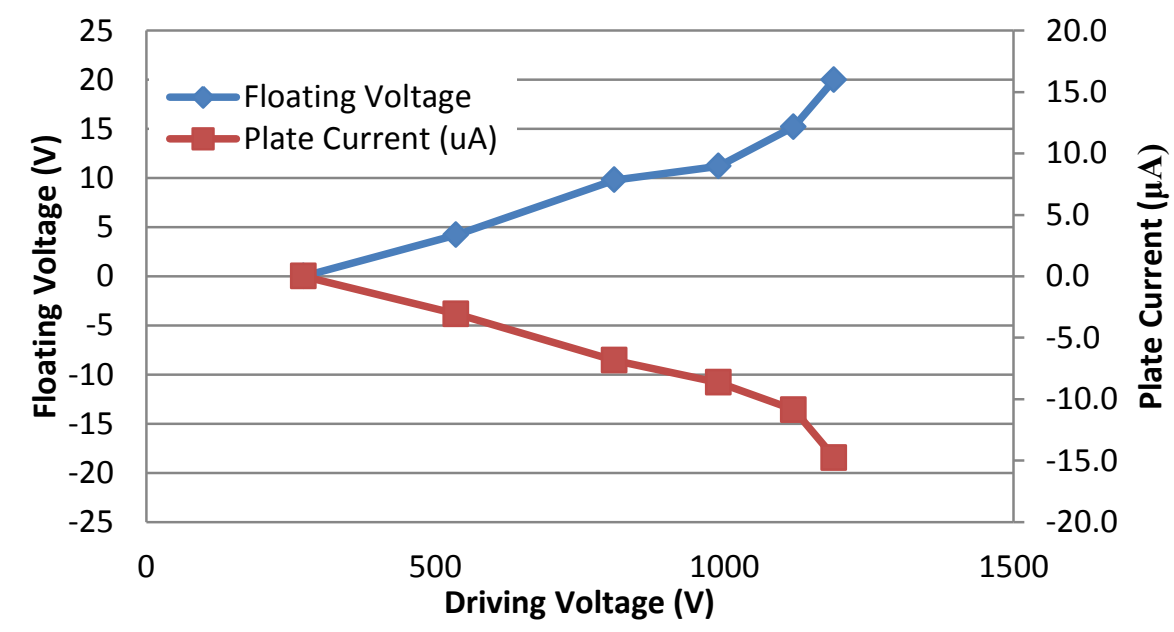

Figure 4.15: Floating voltage of thruster assembly with argon gas and $0 \mathrm{~V}$ extraction.

\subsection{Device Lifetime}

In this section, parameter variations that had an effect on the lifetime performance of the PTPS-MT are discussed. Modes of crystal failure and results from an extended six day continuous operation are provided. 


\subsubsection{Modes of Crystal Failure}

During operation of the PTPS-MT, a number of crystal failures occurred as parameters such as driving voltage and duty cycle, were iteratively increased in an attempt to increase the thruster performance. A total of 13 crystals were damaged during the refinement of operating conditions of the thruster. From these failures, three modes of failure are proposed: harmonic distortion in the applied signal, electrical overdrive, and thermally induced stress.

The first mode of failure resulted from saturation of the impedance matching transformer, resulting in large currents being delivered to the PTPS-MT with harmonic distortion in the applied signal as shown in Figure 4.19b,c. The impedance matching transformer used an Amidon iron powder toroidal core, model T-225-26. The amount of energy that can be stored is limited by the saturation or temperature rise, resulting in core loss. To determine the limits of this design, a comparison of the limiting flux density due to core losses was required. Based on manufacturer specifications, the maximum flux density before saturation would occur was 170 Gauss [36]. The flux density in the lab transformer could be found by:

$$
B_{\text {max }}=\frac{E_{p k^{*}} 10^{8}}{4.44 A_{e} N F}
$$

Where $B_{\text {max }}$ is the maximum attainable flux density, $E_{p k}$ is the applied voltage (RMS), $A_{e}$ is the transformer cross-sectional area $\left(\mathrm{cm}^{2}\right), N$ is the number of wire turns on the primary and $F$ is the applied frequency. The maximum achievable flux based on the lab transformer design and operating conditions was $\sim 200$ Gauss, above the saturation limit 
for most voltages used to drive the crystal with the amplifier operating at $100 \%$. The effect of this saturation is the harmonic distortion seen in Figure 4.16b and 4.16c. This distortion would cause high stress in the crystal due to the rapid changes in the applied voltage, resulting in failure. Therefore, the amplifier was only operated at $80 \%$ of its maximum during early testing to limit transformer saturation and maintain a clean signal to the crystal.

The development of a new impedance matching transformer, used in later experiments, eliminated this distortion by providing better saturation resistance through a larger cross-sectional area, better primary winding design, and greater saturation flux density. Figure $4.16 \mathrm{~d}$ shows the signal at the crystal with the amplifier operating at $100 \%$. This design allowed application of higher input voltages being used with no signal distortion experienced. A comparison between the old and new transformer core properties is given in Table 4.4 .

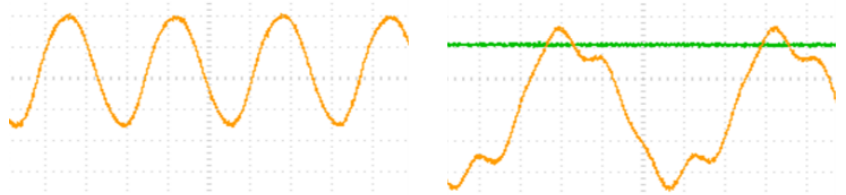

(a)

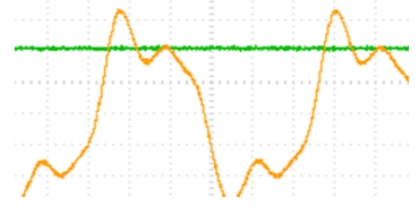

(c) (b)

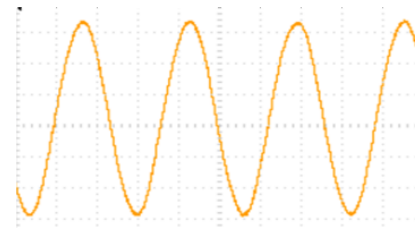

(d)

Figure 4.16: (a) Early transformer operated at 35V (80\% Amplification), (b) same transformer at $90 \%$ amplification, (c) same transformer at 100\% amplification, (d) new transformer design operated at $100 \%$ amplification 
Table 4.4: Comparison of transformer core properties [36].

\begin{tabular}{|l|l|}
\hline \multicolumn{2}{|l|}{ Old } \\
\hline \multicolumn{2}{|l|}{ Amidon T-225-26 } \\
\hline Material & 26 \\
\hline$A_{e}$ & $150.8 \mathrm{~mm}^{2}$ \\
\hline $\mathrm{L}_{\mathrm{e}}$ & $145.6 \mathrm{~mm}$ \\
\hline $\mathrm{V}_{\mathrm{e}}$ & $22685 \mathrm{~mm}^{3}$ \\
\hline Material \\
\hline 26 & \\
\hline $\mathrm{U}_{\mathrm{i}}$ & 75 \\
\hline $\mathrm{f}$ & $60 \mathrm{~Hz}-300 \mathrm{kHz}$ \\
\hline $\mathrm{P}_{\text {diss }}$ & $436 \mathrm{~kW} / \mathrm{m3}$ \\
\hline$B_{\text {sat }}$ & $170 \mathrm{mT}$ \\
\hline
\end{tabular}

\begin{tabular}{|l|l|}
\hline \multicolumn{2}{|l|}{ New } \\
\hline \multicolumn{2}{|l|}{ Epcos PM 50/39 Core } \\
\hline Material: & N87 \\
\hline$A_{e}$ & $370 \mathrm{~mm}^{2}$ \\
\hline$L_{e}$ & $84 \mathrm{~mm}$ \\
\hline$V_{e}$ & $31000 \mathrm{~mm}^{3}$ \\
\hline Material \\
\hline N87 \\
\hline$u_{i}$ & 2200 \\
\hline$f$ & $25-500 \mathrm{kHz}$ \\
\hline$P_{\text {diss }}$ & $380 \mathrm{~kW} / \mathrm{m}^{3}$ \\
\hline$B_{\text {sat }}$ & $390 \mathrm{mT}$ \\
\hline
\end{tabular}

The failures experienced from the signal distortion were noted early in testing and were corrected for by lowering the amplification of the signal. The final data presented in this thesis are based on the newer impedance matching transformer design. Results obtained with the newer transformer greatly enhanced the operation of the PTPS-MT as higher voltages could be applied, resulting in a better ionization of propellant and leading to higher ion currents than were previously achieved.

The electrical overdrive (E-O) failures were the most common, occurring a total of nine times. These failures occurred when the voltage applied to the piezoelectric created internal stresses which surpassed the failure limit of the crystal. Typically this would occur around an applied voltage of $1500 \mathrm{~V}$ in the lab. COMSOL simulations for a radial resonant disc at this voltage indicated stress values reaching over $130 \mathrm{MPa}$. Failure in lithium niobate can occur between 30-120 MPa, depending on the orientation of the 
crystal lattice [26]. The simulated values fall within this range, providing a reasonable assumption that mechanical failure was the result of E-O. A second supporting argument for the E-O failure comes from the location of the highest stress. Operating in the radial resonant mode, the largest stress occurs in the center of the disc as shown in Figure 4.18a. All crystal failures in this category exhibited fractures that would pass through the center of the piezoelectric crystal as shown in Figure 4.18b.

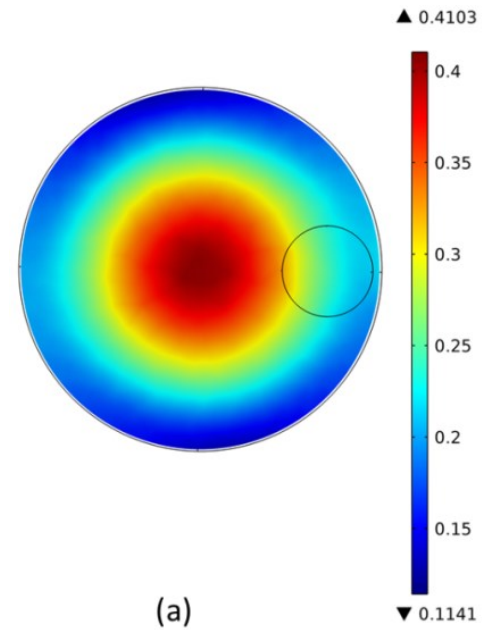

(a)

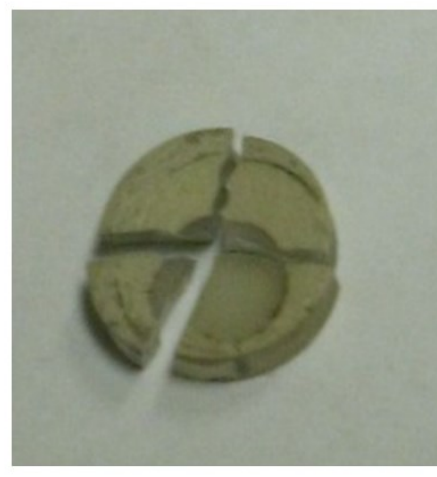

(b)

Figure 4.17: (a) location of highest stress. (b) resulting crystal fracture profile

The third failure mode, thermally induced stress, occurred when the PTPS-MT was operated at high duty cycles and driving voltages. The longer operation, however, resulted in the plasma being exposed to the surface of the disc for extended periods without much of a mechanism present to control the rise in temperature on the surface of the lithium niobate. The extended exposure of the crystal surface to the plasma greatly increased erosion, most likely weakening the lithium niobate. Figure 4.18 shows scanning electron microscope (SEM) photography of a crystal surface after a series of $20 \%$ duty 
cycle tests. Four regions of the crystal surface are highlighted. In region 1, silver paint covers the surface of the crystal but was not exposed to the plasma due to coverage by the grounded electrode. In region 2, the silver paint was exposed to the plasma. Region 3 was the burn ring caused by plasma discharges. The burn ring follows the entire outer edge of the silver paint aperture, which was shown to have an enhanced field generation due to triple point of silver paint, background gas and lithium niobate. Last was region 4, a polished clear center of the aperture most likely as a result of plasma etching.

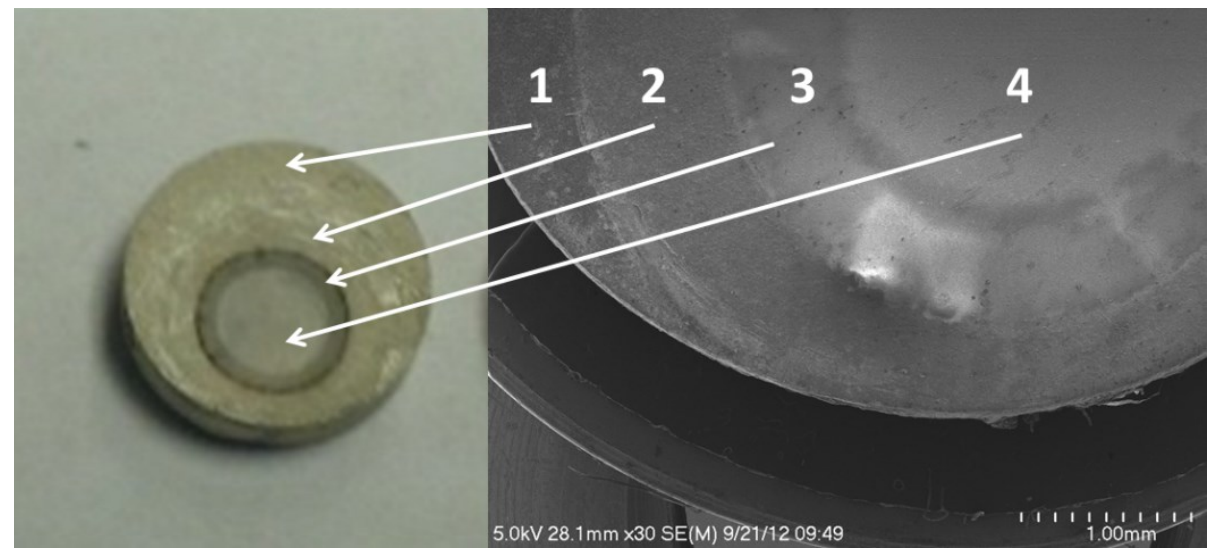

Figure 4.18: Photo and SEM of crystal after prolonged operation showing four regions of interest: (1) electrode covered by ground aperture, (2) painted electrode exposed to plasma chamber, (3) burn ring from location of triple point/ plasma formation, (4) polished center 


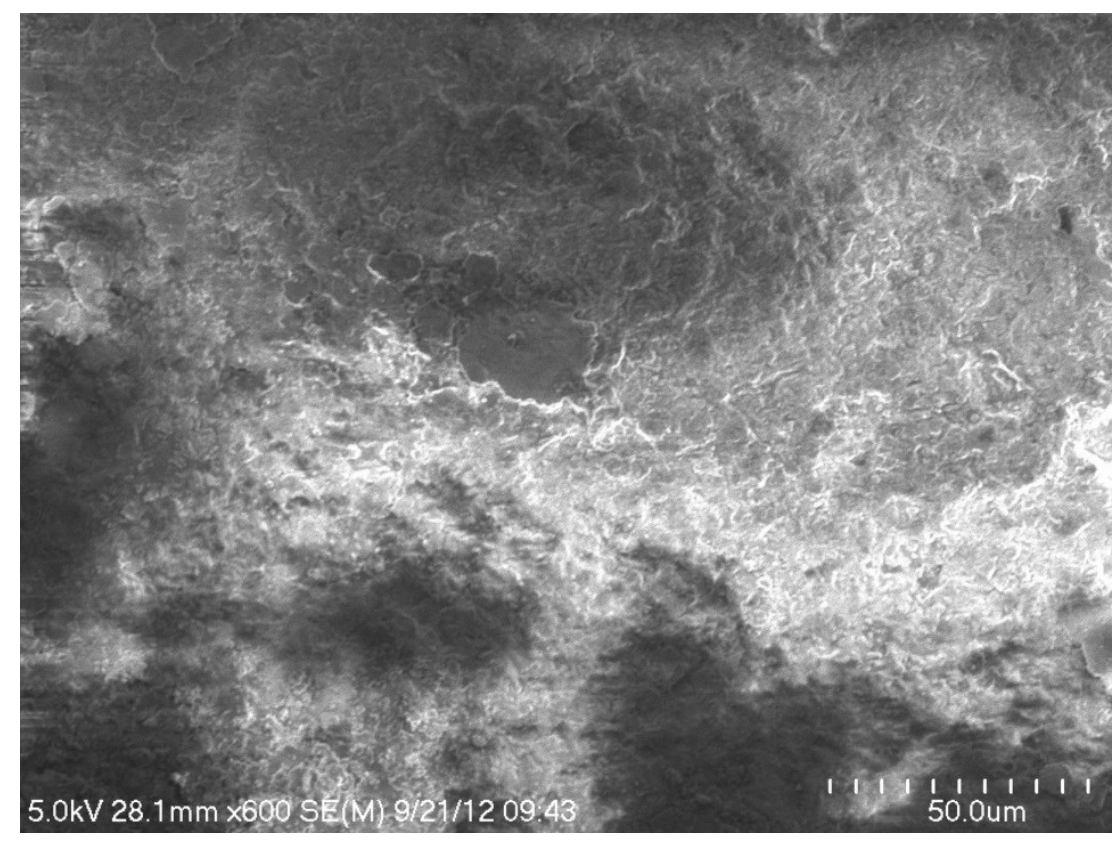

Figure 4.19: Region 3 of crystal showing significant ablation of surface material from plasma discharge at location of triple-point.

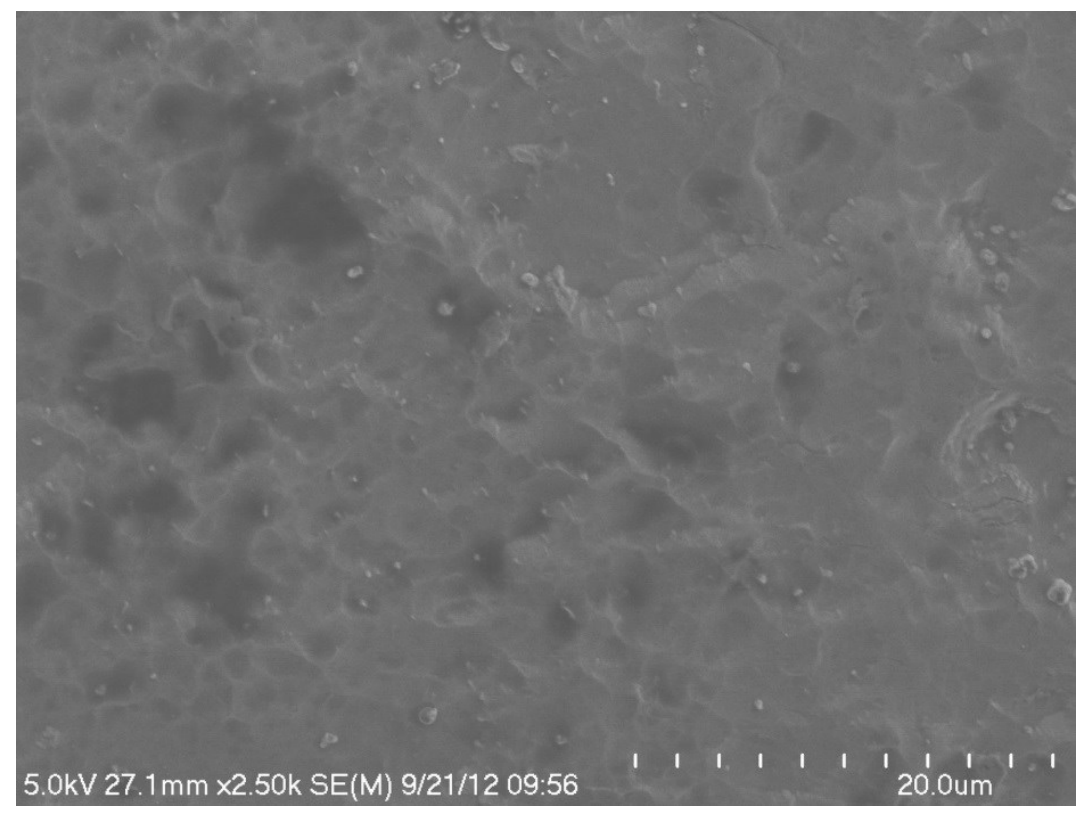

Figure 4.20: Region 4 of crystal. Plasma etching created smooth clear surface on crystal which was opaque and unpolished when new. Presence of surface contaminants, most likely from electrode or silver paint, shown as specks. 
The non-uniform application of heat relative to the surface and base of the crystal would result in the development of a stress gradient being formed. Work by P. Gallagher, in determining the expansion of lithium niobate under that the application of heat, has shown the physical expansion of crystal dimensions by as much $0.2 \%$ for the temperatures measured in the lab [26]. A summary of this work is provided in Table 4.5 for a range of temperatures experienced during the operations of the PTPS-MT. If these expansions are compared to the displacement values found in COMSOL, the equivalent stress found in the crystal becomes larger than the fracture point, as shown in Figure 4.22.

Table 4.5: Radial change in z-cut lithium niobate based on temperature change [26].

\begin{tabular}{|l|l|}
\hline$T(K)$ & $\begin{array}{l}\% \\
\text { Change }\end{array}$ \\
\hline 260 & -0.041 \\
\hline 280 & -0.019 \\
\hline 298 & 0 \\
\hline 320 & 0.025 \\
\hline 340 & 0.048 \\
\hline 360 & 0.072 \\
\hline 380 & 0.098 \\
\hline 473 & 0.222 \\
\hline 573 & 0.360 \\
\hline
\end{tabular}




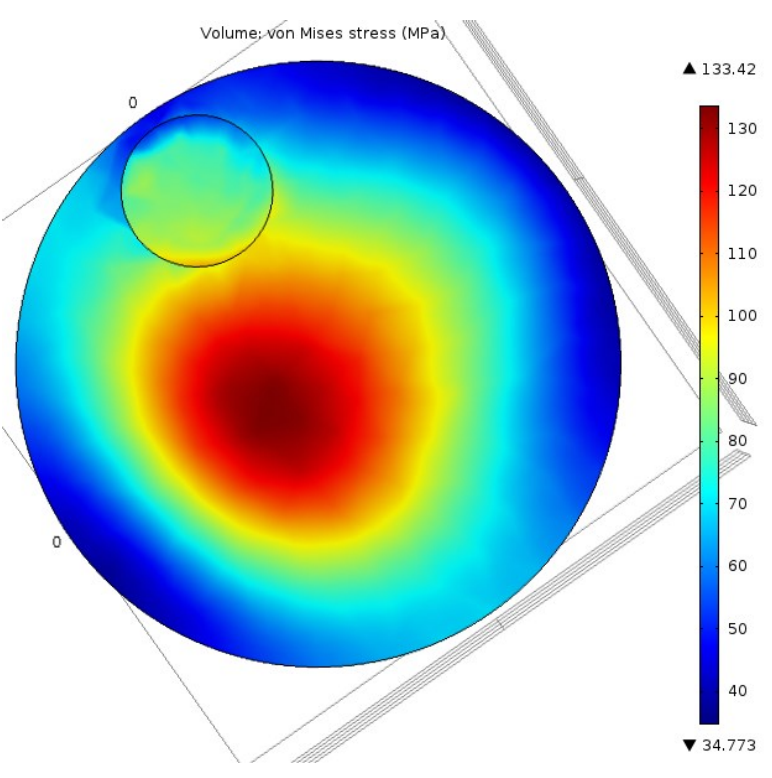

Figure 4.21: Stress profile corresponding to a $0.2 \%$ displacement along radial axis. The maximum stress exceeds the fracture stress for lithium niobate.

\subsubsection{Extended Duration Operation}

Previous work on the PTPS-MT utilized short testing windows where operation of the thruster was done with long burst periods greater than one second and often only for a period of 20 minutes. No work had been done to determine if the PTPS-MT was capable of an extended duration operation that would be required for space applications. A series of two hour tests were initially conducted where the thruster was operated continuously with a 300 cycle burst applied every $50 \mathrm{~ms}$ (1.55\% duty cycle). Thruster performance was measured as the amount of ion current collected on the charge collecting plate at specified intervals over the duration of the test. All data was normalized to the initial ion current, which was $2 \mu \mathrm{A}$, utilizing the early parallel plate thruster design. During this operation period, no crystal failures were experienced. 


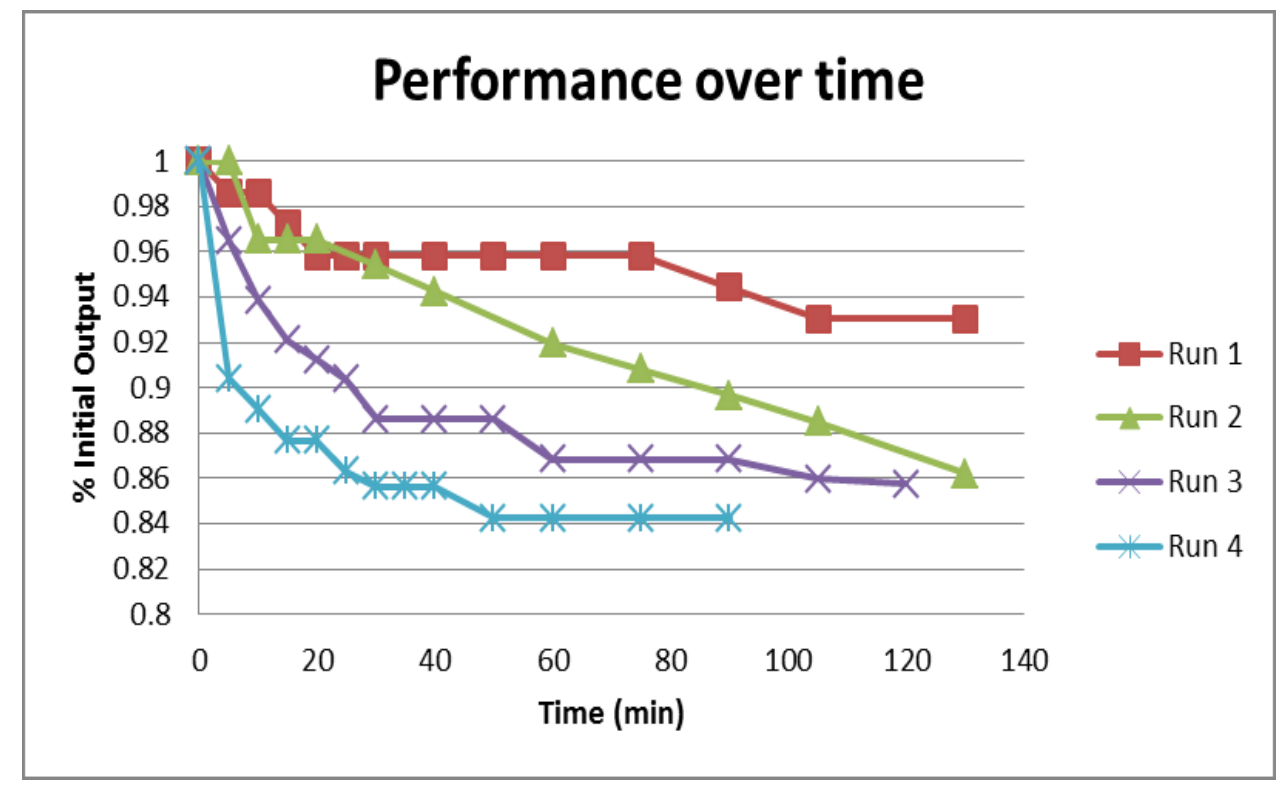

Figure 4.22: Performance of thruster during four two hour testing cycles. Output normalized to peak current of $2 \mu \mathrm{A}$.

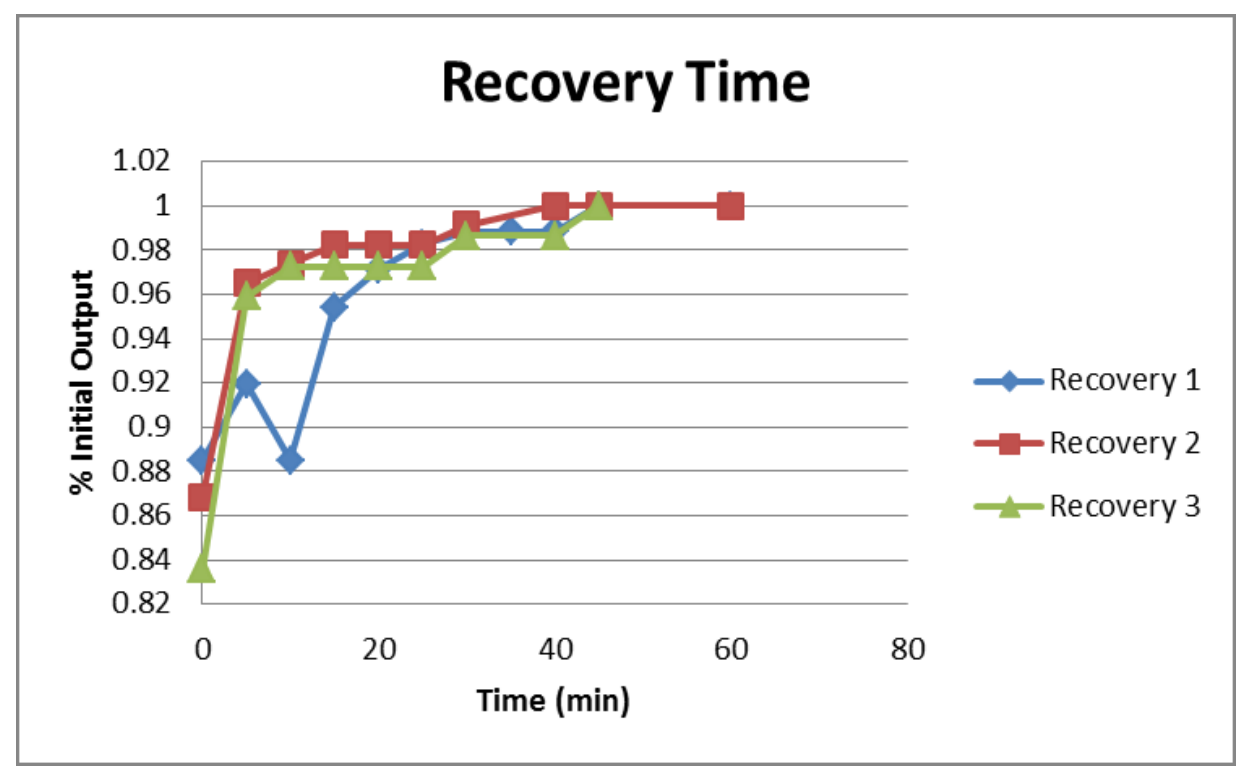

Figure 4.23: Recovery time of thruster to return to initial current output at beginning of two hour run. Each recovery period conducted immediately following 2 hour run with thruster and gas off except for to obtain data point. 
In Figure 4.22 it can be seen that the ion current decreased both during operation and successive testing runs. During the first two hour run the PTPS-MT maintained a nearly steady current output during the entire testing duration. By the third and fourth runs the output rapidly decreased with the fourth run experiencing a $10 \%$ drop in performance in the first 5 minutes. It is believed that this was due to the crystal heating as a result of contact with the plasma over the entire testing period. This reasoning would also explain the recovery time needed to achieve the same output once the crystal had been turned off (Figure 4.23). Despite being operated in a vacuum, cables and housings attached to the crystal would provide a path for heat dissipation over time. Residual heat stored in the apparatus could then manifest during a successive operation run, reducing either the cooling effect it had on the PTPS-MT, or returning a portion of the stored thermal energy back into the system, resulting in decreased performance.

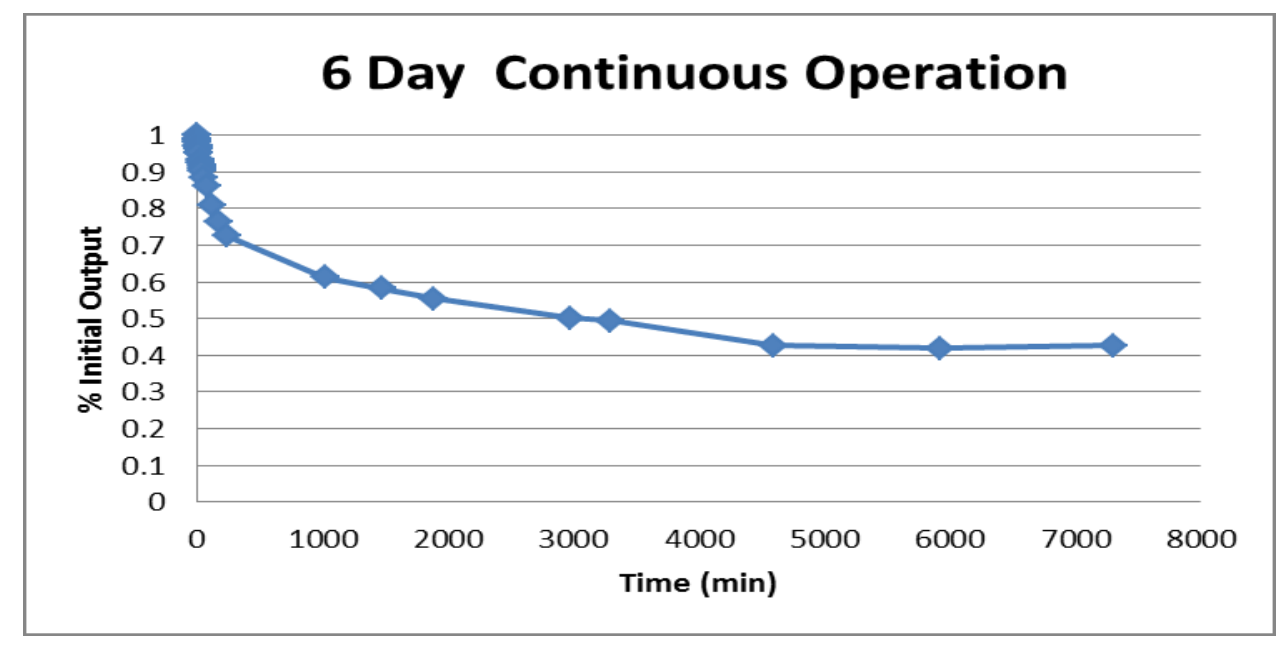

Figure 4.24: Measurement of thruster performance over a six day continuous operation. All operating conditions matched the two hour tests. 
The extended duration test was used to measure performance over a period of days, with operating parameters the same as the two hour tests. As expected from previous testing, the thruster experienced a rapid decline in performance for the initial period. After the first day, a period of slow decline occurred before a steady state of operation began after three days of operation (4600 minutes). The extended run was halted after 6 days due to saturation of the vacuum pump with propellant gas. The same reasoning affecting the shorter duration tests was applied to the decrease in performance over the long term operation. Unlike the shorter two hour tests, the thruster reaches equilibrium and is able to maintain ion current output after this point. The lack of crystal failure during this period shows promise for longer operational periods and demonstrates that thruster design using a piezoelectric crystal may be suitable as a method for space propulsion. 


\section{Chapter 5}

\section{Summary}

\subsection{Conclusions}

Research on the PTPS-MT focused on recommendations in previous works on how to improve the operational efficiency and lifetime performance of the thruster [25]. This included ways to reduce the gas flow, improve the mechanical resonance, and decrease plasma chamber volume in an attempt to improve parameters having an effect on the specific impulse and ion thrust.

Finite-element simulations were used to determine an optimal ground electrode placement and dimensions in an attempt to improve the voltage transformation occurring on the surface of the piezoelectric disc. An increase in the voltage transformation could effectively reduce the size of the power supply by relying on the PTPS-MT to create a high voltage transformation ratio capable of ionizing the propellant gas. In doing so, this could effectively increase the operational payload of a rocket by devoting less mass to large electronics and power systems needed in present day electric propulsion systems. The simulations provided two important results: a standardized ground electrode design that improved the voltage transformation and stress reductions of over $50 \%$ by increasing the size of the piezoelectric disc. By reducing the stress, the chance of failure in the PTPS-MT is decreased resulting in a potentially longer lifetime. Additionally, a location of the ground electrode aperture corresponding to a $70 \%$ offset and diameter of $25 \%$ the disc radius would provide the highest transformation ratio. The improved electrode design increased measured voltages by more than a factor of five, allowing the PTPS-MT 
to be run at voltages less than $200 \mathrm{~V}$ with consistent results, with higher voltages resulting in a higher plasma density.

A new holder for the PTPS-MT was designed, improving the mechanical quality factor over the older parallel plate design by a factor of 10 . With the new holder, a consistent spring force held the crystal in place. The previous design required the physical tensioning of screws against two parallel plates to create a clamping force. The parallel plate tensioning method resulted in a mechanical quality factor of $\sim 50$ while the newer spring clamping method improved the factor to nearly 500. This provided an increased in the stored energy within the PTPS-MT compared to the input power allowing for an increase in efficiency.

The thruster was an improved design aimed at reducing the propellant flow in an attempt to improve the specific impulse of the system. Research conducted by Hutsel and Kemp suffered from extremely high propellant flows to sustain a plasma based on their large chamber design [25], [38]. Despite the significant reduction in propellant used in the PTPS-MT design, specific impulses failed to improve. This was a result of the low ionization and extraction to generate thrust. Future works will have to focus on a design that is able to maintain a low propellant flow while providing a method improve the ionization of propellant gasses.

\subsection{Future Work}

Any future work on the PTPS-MT should focus on increasing the extraction of ions prior to their recombination. This could be approached a few ways. First, application 
of a high magnetic field could be used to contain the plasma which should prevent the effect of the recombination of ions and electrons at the chamber surface. This would also aid in preventing the ablation of the chamber over time and allow a significant portion of the ions to be accelerated out of the aperture compared to the current design. Use of the magnetic field may also aid in the acceleration of the ions to a considerable extent. Works have been published on electromagnetic thrusters which use 1-2 Tesla magnetic fields for acceleration [10]. Neodymium rare earth magnets can provide up to a 1.4 Tesla field in a compact disc on the scale of the current PTPS-MT [37]. Any approach with magnetic confinement will most likely have to look at external neutralization methods as the magnetic fields would continuously contain electrons.

The containment aperture can be approached two ways: acceleration grid or open aperture design. The acceleration grid method could be used to increase the velocity of the ions, thereby increasing the thrust. An excess of electrons would remain inside the chamber if the grid voltage is high enough aiding in further ionization. The drawback of this approach is the significant increase in floating potential since only positive charge is selectively released. It has been noted in the PTPS-MT design that the absence of an accelerator grid results in an excess release of electrons. This effect could be used to counter the ion loss by possibly using a pulsed grid design timed to the cycles of the piezoelectric disc in which the choice of either electron or ion ejection could occur.

Increasing the containment aperture exposes the plasma chamber to vacuum resulting in a faster loss of neutral gas. To help prevent this, the use of microfluidic channels in the ground plate aperture could be used to release the propellant gas onto the 
surface of the piezoelectric. The current design has the gas flow occurring high in the chamber, creating favorable conditions for selectively discharging only neutral gas. The gas flow though the microfluidic channels could also be pulsed, releasing a timed propellant burst in sync with the high voltage peak from the piezoelectric. This design should help in reducing neutral gas loss and aid in the reduction of gas flow by placing the right amount of propellant at the right position at the right time.

Finally, use of a larger crystal would allow for higher applied voltages and transformation ratios, which could be used to obtain larger percentages of ionization. The larger crystal in simulations has shown a reduction in both stress and increase in the transformation ratio, making it desirable for future designs.

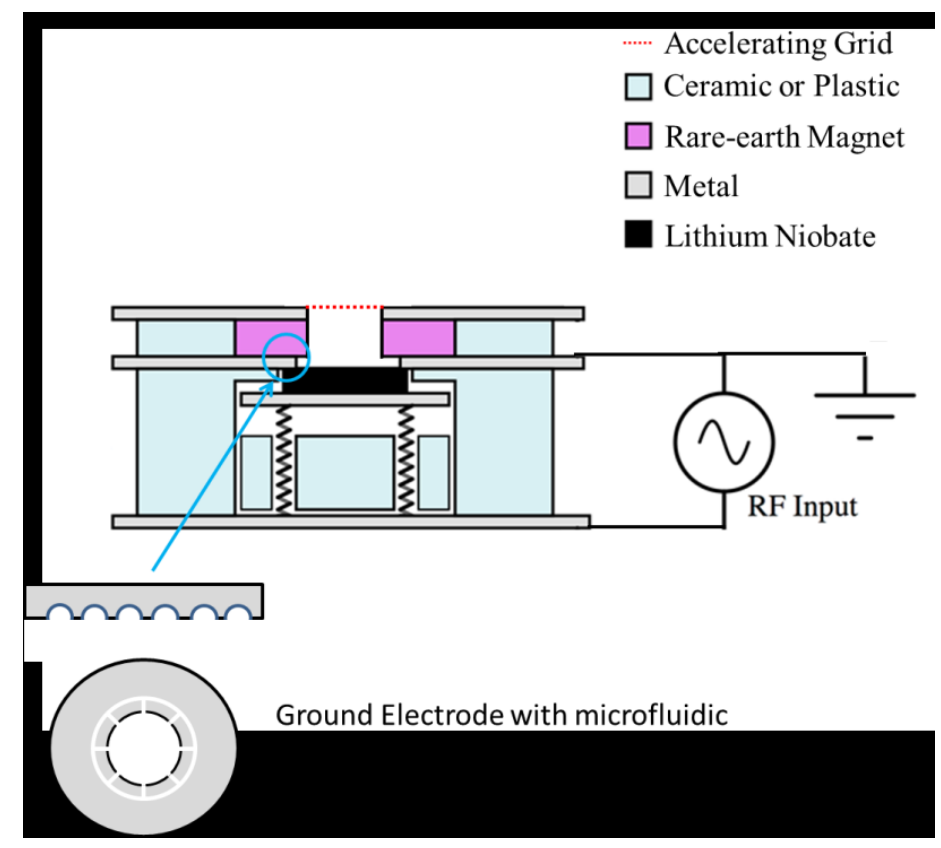

Figure 5.1: Recommended thruster design for any future approaches to the PTPS-MT. 


\section{APPENDIX A}

\section{CRYSTAL PREPARATION}

Proper preparation of the crystal is important in order to achieve a high mechanical quality factor, high voltage transformation ratio, and uniform discharge. The procedures here are provided as best practice guidelines in order to help maintain consistent results between crystals.

Step 1: Identify polarity using heat source and voltage monitor. Placing one tip of the voltage monitor on top of the crystal and the second on the metal hot plate, either a positive or negative voltage will appear. Depending on the sign, you can now determine the polarity of the crystal. Mark the positive side for reference.

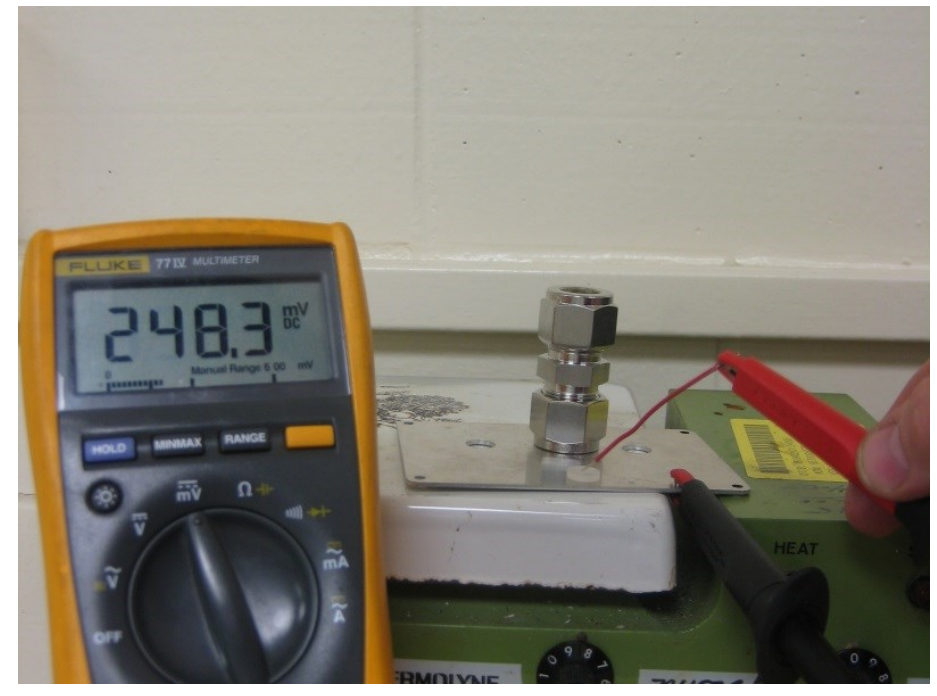

Figure A.1: Step 1 reference photograph

Step 2: Determine polarization direction. This process involves a series of preparation steps in order to obtain a plasma discharge needed to identify the polarization direction. 
Step 2a: Paint negative polarity side of crystal with silver paint. Let dry.

Step 2b: Paint small ring of silver paint along outer edge of surface to act as an electrode, shown below. Let dry completely.

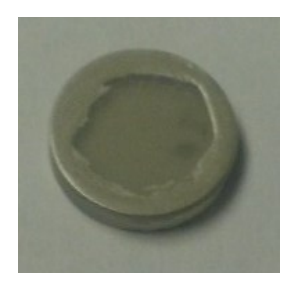

Figure A.2: Step 2b reference image

Step 2c: Place crystal in holder and apply RF voltage until plasma discharge occurs. This may require slight adjustments in frequency as each crystal is slightly different. It is best to start at $380 \mathrm{kHz}$ and work up by $100 \mathrm{~Hz}$ each step to find the resonance/ plasma discharge.

Step 2d: Once plasma discharge is occurring, mark the center of the discharge crescent with a lead pencil. This marks the polarization direction and is the location you will place the aperture offset.

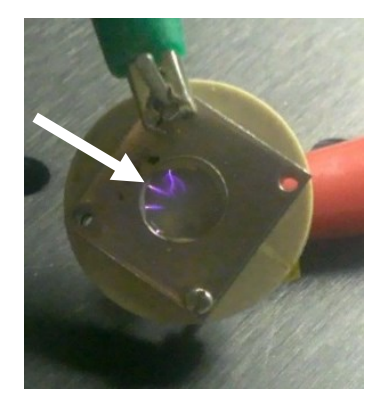

Figure A.3: Step 2d reference image 
Step 3: Remove crystal from holder and mark the edge of the crystal where the center of the plasma formed. This will help guide the aperture offset after painting the surface.

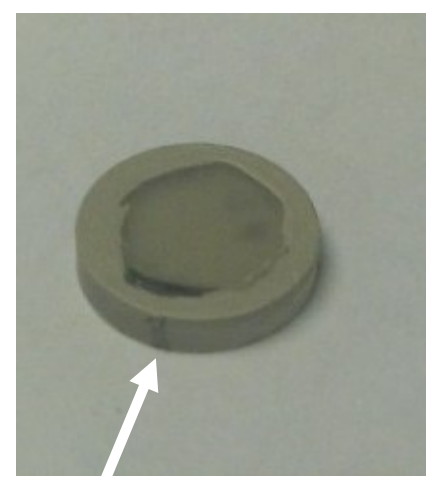

Figure A.4: Step 3 reference image

Step 4: Remove the silver paint ring from the surface of the crystal and then repaint completely. Removing the ring prevents excess paint buildup and will allow for a smooth surface.

Step 5: Create an aperture mask using Kapton tape and a punch corresponding to desired aperture diameter. For $10 \mathrm{~mm}$ diameter crystals, the punch should be $2.5 \mathrm{~mm}$.

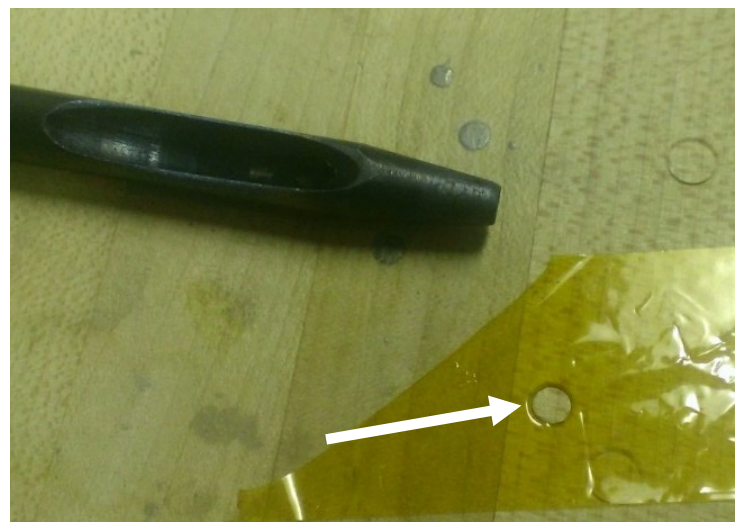

Figure A.5: Step 5 reference image 
Step 6: Place Kapton tape on crystal suface corresponding to offset in direction of plasma discharge from step 2. This should be where your pencil mark is. For $10 \mathrm{~mm}$ crystal, centered offset should be $3.5 \mathrm{~mm}$ to center of Kapton tape aperture (there should be a small ring of silver paint along the outside of crystal.)

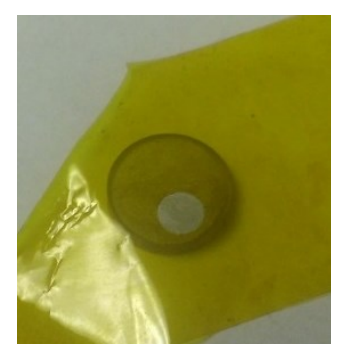

Figure A.6: Step 6 reference image

Step 7: Remove silver paint from aperture using paint thinner.

Step 8: Lightly sand both surfaces of painted crystal to allow for uniform contact with electrodes using 600 grit or higher sandpaper. Do not sand off silver paint completely.

Step 9: Place crystal in holder and apply RF voltage. There should be a uniform circular discharge across the entire aperture as seen below:

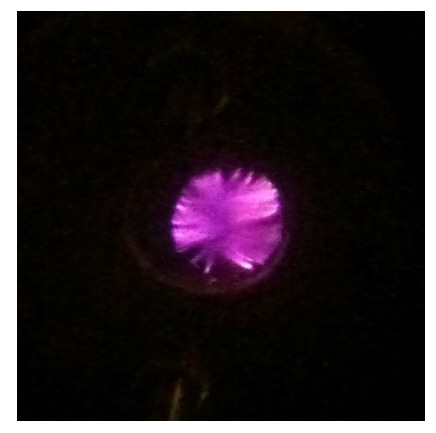

Figure A.7: Step 9 reference image 


\section{APPENDIX B}

\section{GAS FLOW CALCULATIONS}

The mass flow controller used in the measurement of gasses was calibrated to $30 \mathrm{sccm}$ for nitrogen gas. Flow rates of other gases could be calculated using manufacturer provided correction factors provided below:

Table B.1: Gas correction factors for mass flow controller

\begin{tabular}{|l|l|l|}
\hline Gas & $\begin{array}{l}\text { Correction } \\
\text { Factor }\end{array}$ & $\begin{array}{l}\text { Maximum } \\
\text { Available } \\
\text { Flow }\end{array}$ \\
\hline Nitrogen & 1 & $30 \mathrm{sccm}$ \\
\hline Hydrogen & 1.01 & $30.3 \mathrm{sccm}$ \\
\hline Argon & 1.45 & $43.5 \mathrm{sccm}$ \\
\hline Xenon & 1.44 & $43.2 \mathrm{sccm}$ \\
\hline
\end{tabular}

Calculation of mass flow in $[\mathrm{kg} / \mathrm{s}]$ was done according to:

$$
\dot{m}=\frac{m P}{n R T} \dot{V}
$$

wherem is the mass flow $(\mathrm{kg} / \mathrm{s}), \dot{V}$ is the volumetric flow (sccm), $m$ is the ion mass $(\mathrm{g} / \mathrm{mol}), P$ is the gauge pressure at the MFC inlet, $n$ is the number of moles of gas, $R$ is the universal gas constant, and $T$ is the temperature of the gas. Calibration of the MFC used in the lab was done at 15 PSI gauge pressure and must be the pressure used to maintain calibrated flow rates.

The chamber pressure could be calculated using small aperture approximation and the assumption that the vacuum chamber was at ideal vacuum. The pressure based on the 
flow rate would then correspond to the pressure found in the plasma chamber, according to:

$$
P=\frac{4 Q}{v_{t h} A_{p}}
$$

where

$$
v_{t h}=\sqrt{\frac{8 k T}{\pi m}},
$$

$Q$ is the volumetric flow rate and $A p$ is the orifice aperture diameter between the two pressure differentials (vacuum chamber and plasma chamber in this case). 


\section{BIBLIOGRAPHY}

[1] "Electric Propulsion," in in Encyclopedia of Physical Science and Technology, Elsevier, 2004, pp. 125-141.

[2] G. Puskulcu, B. Sumer, D. E. Gunduz, C. Yildirim, B. Yazici, F. E. Orhan, L. O. Gonc, and M. A. Ak, "Ground testing of a liquid fueled rocket motor," pp. 319-323.

[3] D. K. Huzel, D. H. Huang, and H. Arbit, Modern engineering for design of liquidpropellant rocket engines. Washington, D.C.: American Institute of Aeronautics and Astronautics, 1992.

[4] R. G. Jahn, Physics of electric propulsion. Mineola, N.Y: Dover Publications, Inc, 2006.

[5] S. K. Borowski, D. R. McCurdy, and T. W. Packard, "Nuclear Thermal Propulsion (NTP): A proven growth technology for human NEO/Mars exploration missions]]>," 2012, pp. 1-20.

[6] Fundamentals of solid-propellant combustion. New York, N.Y: American Institute of Aeronautics and Astronautics, 1984.

[7] P. Balachandran, Fundamentals of compressible fluid dynamics. New Delhi: Prentice-Hall of India, 2007.

[8] North Atlantic Treaty Organization and NATO Advanced Research Workshop on Electron Kinetics and Applications of Glow Discharges, Electron kinetics and applications of glow discharges. New York: Plenum Press, 1998.

[9] P. J. Wilbur, R. G. Jahn, and F. C. Curran, "Space electric propulsion plasmas," IEEE Transactions on Plasma Science, vol. 19, no. 6, pp. 1167-1179, Dec. 1991.

[10] M. Patterson, "NEXT Ion Propulsion System Development Status and Capabilities," presented at the NASA Science and Technology Conference, 2007.

[11] J. R. Anderson, K. D. Goodfellow, J. E. Polk, V. K. Rawlin, and J. S. Sovey, "Performance characteristics of the NSTAR ion thruster during an on-going long duration ground test," vol. 4, pp. 99-122.

[12] L. Frantz, D. Geffen, C. Hendricks, J. Lair, D. Langmuir, G. Nazarian, J. Sellen, H. Shelton, P. Stehle, H. Wachowski, and R. Wuerker., "Electrostatic Propulsion," Proceedings of the IRE, vol. 48, no. 4, pp. 477-491, Apr. 1960. 
[13] D. C. Byers and B. A. Banks, "Survey of Electron-Bombardment Thruster Research," IEEE Transactions on Plasma Science, vol. 1, no. 2, pp. 1-9, Jun. 1973.

[14] J. E. Polk, M. J. Sekerak, J. K. Ziemer, J. Schein, Niansheng Qi, and A. Anders, "A Theoretical Analysis of Vacuum Arc Thruster and Vacuum Arc Ion Thruster Performance," IEEE Transactions on Plasma Science, vol. 36, no. 5, pp. 2167-2179, Oct. 2008.

[15] J. R. Beattie and J. N. Matossian, "Xenon ion sources for space applications (invited)," Review of Scientific Instruments, vol. 61, no. 1, p. 348, 1990.

[16] "NASA Facts," 2008. [Online]. Available: https://www.nasa.gov/centers/glenn/pdf/84795main_fs08grc.pdf. [Accessed: 11Apr-2013].

[17] R. T. Downey, P. Giuliano, K. D. Goodfellow, and D. Erwin, "Single-Channel Hollow Cathodes in 5-20-eV Argon Discharge for Spacecraft Thruster Applications," IEEE Transactions on Plasma Science, vol. 39, no. 4, pp. 1075-1081, Apr. 2011.

[18] M. Lapointe and P. Mikellides, "High-Power Electromagnetic Thruster Being Developed." Glenn Research Facility.

[19] D. A. Codron and D. Erwin, "Experimental studies on high current arc discharges for magnetoplasmadynamic thrusters," 2012, pp. 1-9.

[20] T. Ikeda, Fundamentals of piezoelectricity, Corrected pbk. ed. Oxford ; New York: Oxford University Press, 1996.

[21] L. K. Erickson, Space flight: history, technology, and operations. Lanham, Md: Government Institutes, 2010.

[22] G. P. Sutton, Rocket propulsion elements, 8th ed. Hoboken, N.J: Wiley, 2010.

[23] F. A. Zotes and M. S. Penas, "Delta-V genetic optimisation of a trajectory from Earth to Saturn with fly-by in Mars," 2010, pp. 1-6.

[24] Microsoft Encarta Encyclopedia. 2000.

[25] B. Hutsel, "Characterization of a piezoelectric transformer plasma source," University of Missouri, 2012. 
[26] K. K. Wong, Institution of Electrical Engineers, and INSPEC (Information service), Properties of lithium niobate. London: INSPEC/Institution of Electrical Engineers, 2002.

[27] S. Kim, V. Gopalan, and A. Gruverman, "Coercive fields in ferroelectrics: A case study in lithium niobate and lithium tantalate," Applied Physics Letters, vol. 80, no. 15, p. 2740, 2002.

[28] G. Ivensky, I. Zafrany, and S. Ben-Yaakov, "Generic operational characteristics of piezoelectric transformers," IEEE Transactions on Power Electronics, vol. 17, no. 6, pp. 1049-1057, Nov. 2002.

[29] COMSOL Multiphysics ver 4.2. Burlington, MA: COMSOL.

[30] L. APC International, Piezoelectric ceramics: principles and applications. Mackeyville, PA: APC International, 2011.

[31] D. M. Goebel, Fundamentals of electric propulsion: ion and Hall thrusters. Hoboken, N.J: Wiley, 2008.

[32] F. F. Chen, Introduction to plasma physics and controlled fusion, 2nd ed. New York: Plenum Press, 1984.

[33] N. E. Todreas, Measurement and detection of radiation. [S.I.]: Brunner-Routledge, 1995.

[34] V. D. da Silva, Mechanics and strength of materials. Berlin ; New York: Springer, 2006.

[35] A. Kruithof, Townshend's ionization coefficients for neon, argon, krypton and xenon, Physica. Vol 7. Iss. 6

[36] Amidon, Inc. Santa Ana, CA. 1995.

[37] Magnet properties, available from: http://www.rare-earthmagnets.com/?utm_source=bing\&utm_medium $=$ cpc\&utm_term=Neodymium\%2B Magnets\&utm_campaign=National\%2BImports

[38] M. Kemp, "The Ferroelectric Plasma Thruster," University of Missouri, 2008.

[39] Sutton, G., Rocket Propulsion Elements. 2001. Wiley and Sons, Inc.

[40] http://sdmomfia.com/wp-content/uploads/2011/06/mylittlepony.jpg 